\title{
Electron-Deuteron Scattering with HERA, a Letter of Intent for an Experimental Programme with the H1 Detector
}

\author{
List of supporting physicists and institutes to be added
}

\begin{abstract}
This document outlines the case for a programme of electron-deuteron scattering measurements at HERA using the $\mathrm{H} 1$ detector. The goals of the $e^{ \pm} D$ programme are to map the partonic structure of the nucleon at large $Q^{2}$ and low $x$, to explore the valence quark distributions at the highest $x$ values, to provide a precise measurement of the strong coupling constant and to investigate the parton recombination phenomena revealed in shadowing and their relationship to diffraction. The importance of these measurements for the understanding of the perturbative and non-perturbative aspects of QCD thought to be responsible for nucleon structure is discussed, as is the significance of the measurements for future experimental programmes. Some modifications to both the H1 apparatus and the HERA accelerator are necessary to realise this programme; these are presented in the document. Mention is also made of questions that will remain unanswered following the completion of the above programme and the potential rôle of HERA and of $\mathrm{H} 1$ in investigating these questions is outlined.

Physicists and Institutes interested in supporting this project are asked to inform Max Klein (klein@ifh.de) and Tim Greenshaw (green@hep.ph.liv.ac.uk) that they would like to have their names on the Letter of Intent by Wednesday $30^{\text {th }}$ April 2003.
\end{abstract}




\section{Contents}

1 Introduction 3

2 Electron Deuteron Scattering at HERA 4

2.1 Introduction . . . . . . . . . . . . . . . . . . . . 4

2.2 Structure Functions and Parton Distributions . . . . . . . . . . . . . . . 7

2.2.1 Flavour asymmetry of sea quarks at low $\ldots \ldots \ldots$

2.2.2 Valence quarks at large $x \ldots \ldots \ldots \ldots$

2.2.3 Parton distributions and measurement of the strong coupling constant . 13

2.3 Nuclear shadowing in deuterium . . . . . . . . . . . . . . . . . 17

2.3.1 Tagging spectator protons and neutrons . . . . . . . . . . . 18

2.3.2 Simultaneous tagging of protons and neutrons . . . . . . . . . 23

2.4 Diffraction . . . . . . . . . . . . . . . . . . 23

2.4.1 Coherent diffraction from the deuteron . . . . . . . . . . . . . 24

2.4.2 Comparisons of diffraction from the neutron and the proton . . . . . 25

2.4 .3 Vector meson production . . . . . . . . . . . . . . . . . 29

3 Low $x$ Physics and QCD at High Parton Density 30

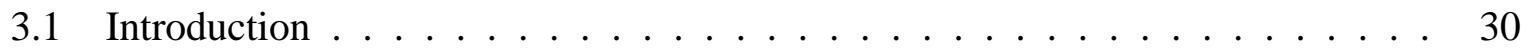

3.2 Gluons at low $x$ and $Q^{2}$ and the measurement of $\boldsymbol{F}_{L}^{p} \ldots \ldots \ldots$

3.3 Measurements in the forward region . . . . . . . . . . . . 35

3.3 .1 Parton branching . . . . . . . . . . . . . . . 35

3.3 .2 The QCD vacuum . . . . . . . . . . . . . . . 37

3.3.3 Non-perturbative particle production . . . . . . . . . . . . 37

3.3.4 The experimental situation . . . . . . . . . . . . . . . 39

3.3.5 Forward jet measurement . . . . . . . . . . . . . . . 39

3.3.6 Further measurements in the forward region . . . . . . . . . . . . . . 41

3.4 A 3D picture of the proton - generalised parton distributions $\ldots \ldots$. . . . . 41

3.5 Nuclear parton distributions and saturation . . . . . . . . . . . . . . . . . 43

3.5.1 Inclusive nuclear shadowing . . . . . . . . . . . . . . . . . . . 44

3.5.2 Inclusive hadron observables . . . . . . . . . . . . . . . . . . 47

3.5.3 Diffractive observables . . . . . . . . . . . . . . . . 47

3.5.4 Exclusive deep inelastic diffraction . . . . . . . . . . . . . 48 
4 Mapping nucleon and photon spin $\quad 49$

4.1 Measuring the spin structure of longitudinally polarised nucleons . . . . . . . . 49

4.2 Polarised electron-deuteron scattering and shadowing effects . . . . . . . . . 52

4.3 Charged current interactions and flavour dependent spin structure . . . . . . . 52

4.4 Physics with transverse nucleon polarisation . . . . . . . . . . . . . 55

4.5 Photon spin . . . . . . . . . . . . . . . . . . 56

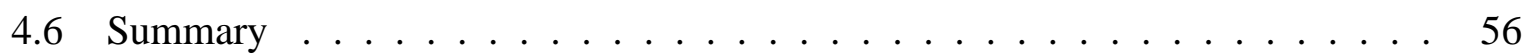

5 Upgrades to the H1 apparatus

5.1 Introduction . . . . . . . . . . . . . . . . . . . 56

5.2 Detecting spectator nucleons, an upgrade of the $\mathrm{H} 1$ forward tagging system . . 57

5.3 The proton spectator tagger . . . . . . . . . . . . . . 58

5.4 Upgrade to the forward region in $\mathrm{H} 1 \ldots \ldots \ldots$

5.4 .1 Introduction . . . . . . . . . . . . . . . . 63

5.4 .2 Preshower detectors $\ldots \ldots \ldots \ldots 6$

5.4 .3 Digital calorimeter . . . . . . . . . . . . . . 68

5.5 Upgrade to the backward region . . . . . . . . . . . . . . . . . 70

5.5.1 Physics requirements . . . . . . . . . . . . . . . 70

5.5.2 Modifications to the $\mathrm{H} 1$ backward region . . . . . . . . . . . . 71

5.5.3 The very backward spectrometer . . . . . . . . . . . . . . 71

5.5.4 Very backward silicon tracking . . . . . . . . . . . . . . . . . 74

5.5.5 The very backward proportional chamber . . . . . . . . . . 75

5.5.6 The very backward electromagnetic SpaCal . . . . . . . . . . . 75

5.5.7 Backward spectrometer summary _. . . . . . . . . . . . . 78

6 Future HERA operation $\quad 78$

6.1 Introduction . . . . . . . . . . . . . . . . . . 78

6.2 Deuterons in HERA . . . . . . . . . . . . . . . . . . . . 78

6.3 Ions in HERA . . . . . . . . . . . . . . . . . . . . . . . . . . 79

6.4 Polarised protons and deuterons in HERA . . . . . . . . . . . . . . 80

6.4 The source . . . . . . . . . . . . . . . . 80

6.4.2 Maintaining polarisation during acceleration . . . . . . . . 81

6.4 .3 Protons ....................... 81

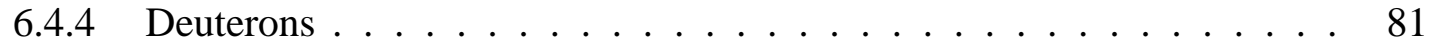

6.4.5 Manipulating deuteron polarisation at the top energy . . . . . . . . 82

6.4.6 Manipulating deuteron polarisation in LINAC-III, DESY-II and PETRA 83

6.4 .7 Polarimetry . . . . . . . . . . . . . . . . 83

$\begin{array}{llr}7 & \text { Summary } & 84\end{array}$ 


\section{Introduction}

Quantum Chromo-Dynamics (QCD) is one of the cornerstones of the Standard Model (SM), and purports to explain the strong interactions that occur between hadrons. As such, it is of enormous significance for our understanding of the Universe; approximately $99 \%$ of baryonic mass is thought to arise through the dynamics of the strong interaction. Many years of experimental and theoretical effort have shown us that QCD is an extremely rich theory. New calculational techniques have been developed such as Lattice Gauge Theory and Heavy Quark Effective Theory and highly sophisticated perturbative QCD (pQCD) calculations are now being carried out to NNLO. Deep inelastic scattering and other experiments have provided a wealth of new information, for example they have revealed that the density of gluons in the proton is huge and discovered the phenomenon of hard diffraction. Despite this experimental and theoretical progress, QCD is in some respects the area of the SM that is least well understood: the precision with which the strong coupling constant is known is notably poorer than that of the couplings in the electromagnetic and weak sectors; the mechanism that ensures quarks are confined within hadrons is not known and it is still not possible to relate measurements of hadronic structure to the fundamental theory. Developing a deeper understanding of QCD therefore remains one of the primary goals of, and one of the most fascinating tasks for, particle physicists.

How can progress be made in a situation in which our level of understanding is such that no set of "definitive" measurements can be proposed which will answer the many remaining questions about QCD? Part of the answer, towards which further steps are presented here, is to extend the experimental data available, both in their nature and their precision, so that they provide ever stronger guidance to those attempting to describe strong interactions in terms of QCD. This programme involves performing new measurements of hadron structure in the regions in which current data indicate our understanding is poorest, in some cases where the nonperturbative aspects of QCD, including the confinement mechanism, may have a significant rôle to play. New data will also be required in areas in which current measurements hint at puzzles that require further investigation; the data from the first phase of HERA running, "HERA I", suggest that there is a new layer to proton structure, at a scale of about a third of the proton's diameter. Further aspects of the possible future programme involve obtaining data on the behaviour of quarks and gluons in new regions, such as at high density in nuclei. Investigations of the relationship between the spin carried by the nucleon and the spin and angular momentum of its constituents may also be carried out in completely new kinematic regions. Experimentally, HERA has so far been used to investigate only the interactions of protons with electrons and positrons. The potential of the facility has thus in no way been exhausted. Collisions with ions, variations of the beam energy and the possibility of nucleon polarisation remain to be explored.

This Letter of Intent expresses the wish of the authors to use the HERA collider and the H1 experiment to pursue the next step in the programme sketched above following the completion of the current "HERA II" running period. The investigation of the interactions of electrons and positrons with deuterons at high energy will make possible the precise measurement of the contributions of the different quark flavours to nucleon structure and of the strong coupling constant. Direct investigations of some of the non-perturbative effects that may influence nucleon structure can also be made, as can new studies of diffraction and of the dynamics of gluon radiation. Section 2 describes this part of the above programme in more detail. This new phase of HERA operation, "HERA III", could be started soon after the completion of HERA II running with 
minimal upgrades to the $\mathrm{H} 1$ detector, or after a shutdown in which more extensive upgrades to the detector and possibly modifications to the $\mathrm{H} 1$ interaction region were made. Where appropriate, reference is made to possible extensions of the programme beyond the electron-deuteron running, including studies of low $x$ physics and QCD at high parton densities, discussed in section 3, and of nucleon spin, described in section 4.

Section 5 describes the possible upgrades to the $\mathrm{H} 1$ detector. One of these, the addition of a new spectrometer to measure protons exiting the $\mathrm{H} 1$ detector with approximately half the deuteron beam energy, is essential for the deuteron programme and can be performed with the current HERA II optics, i.e. with the superconducting magnets in place within H1, or with the optics that pertained for HERAI running, i.e. without superconducting magnets. The other upgrades require the removal of the superconducting magnets and would make possible the low $x$ extension to the deuteron programme, including electron-ion scattering as discussed in section 3. The spin programme would benefit from the high luminosities attainable with the superconducting magnets in place. Detailed planning of how these various possibilities may best be realised must await developments at DESY and elsewhere. The last section of this Letter of Intent, section 6, describes some of the modifications that must be made to HERA to realise both the deuteron programme and the extensions to it mentioned above.

The current efforts of the H1 and ZEUS Collaborations are directed towards obtaining the highest possible precision in measurements at the lepton-quark energy frontier, in probing proton structure down to length scales of $10^{-3} \mathrm{fm}$ and in studies of jet and heavy flavour production using the integrated luminosity of $1 \mathrm{fb}^{-1}$ that will be provided by the upgraded HERA collider. These studies may lead to major new discoveries, in which case the future HERA programme would have to be reconsidered.

\section{Electron Deuteron Scattering at HERA}

\subsection{Introduction}

Our current level of understanding of the high energy interactions of the deuteron, and hence of the neutron, lags dramatically behind that of the proton, as is illustrated by our knowledge of the total cross sections [1], shown in figure 1. A programme of electron-deuteron $(e d)$ scattering at HERA would allow measurement of the $\gamma D$ and $\gamma n$ cross sections up to centre-of-mass energies of 320 and $160 \mathrm{GeV}$ respectively, more than an order of magnitude above the current limits, improve our understanding of the structure of the neutron, one of the most prevalent hadrons in the universe, and provide new data for studies of QCD.

We consider first the deep inelastic scattering (DIS) of electrons and positrons with protons, then move on to discuss what can be learnt through the study of ed scattering. Some of the kinematic variables used in the description deep inelastic ep interactions are shown in figure 2 . The electron interacts with a quark in the proton via the exchange of a virtual photon or $Z$ boson which carries momentum $q=k-k^{\prime}$, where $k$ is the four-momentum of the incident and $k^{\prime}$ that of the outgoing electron. The larger this momentum, the smaller the region probed within the nucleon, the scale generally being described by the variable $Q^{2}=-q^{2}$. Further quantities 


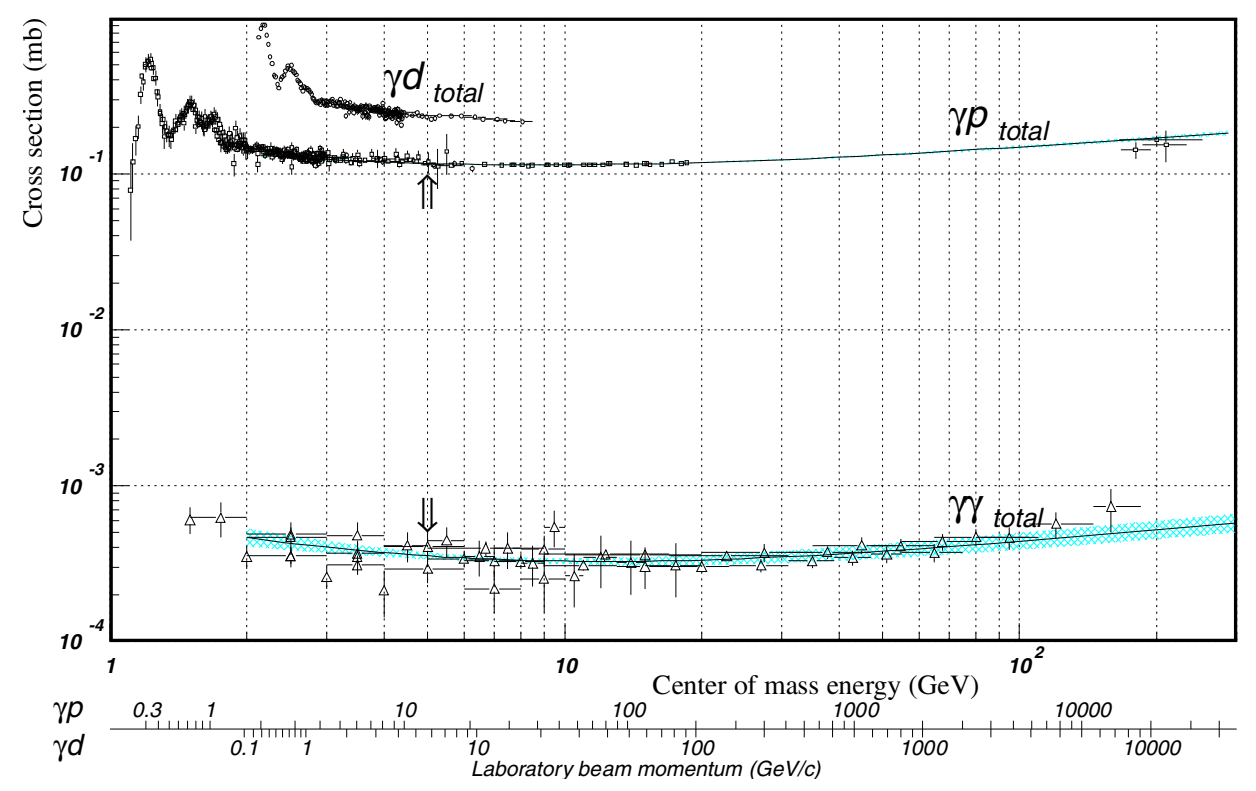

Figure 1: Current measurements of the $\gamma p, \gamma D$ and $\gamma \gamma$ cross sections as a function of centre-ofmass energy [1].

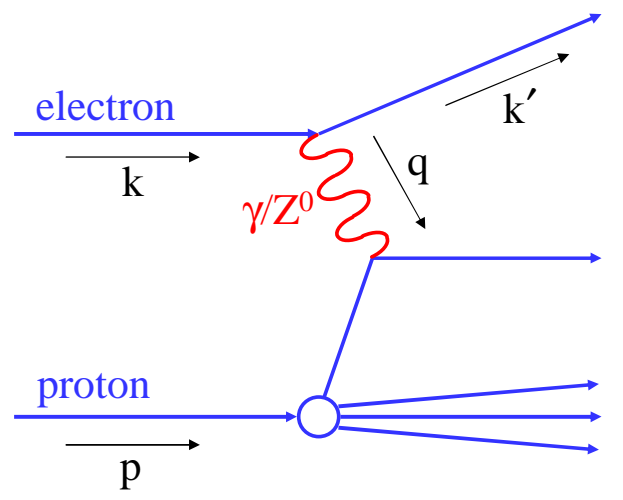

Figure 2: A diagram illustrating some of the kinematic variables used to describe deep inelastic electron-proton scattering. 
used to describe DIS are the Bjorken scaling variable $x=\frac{Q^{2}}{2 p \cdot q}$ and $y=\frac{p \cdot q}{p \cdot k}$. Bjorken $x$ is the proportion of the proton's momentum carried by the struck quark in a frame in which the proton's momentum is large. The quantity $y$ describes the proportion of its energy the electron loses in the scatter, in the frame in which the proton is at rest. The HERA electron and proton beam energies of $E_{e}=27.5 \mathrm{GeV}$ and $E_{p}=920 \mathrm{GeV}$, respectively, have allowed access to the approximate ranges $10^{-5}<x<0.7$ and $0<Q^{2}<30000 \mathrm{GeV}^{2}$. The latter demonstrates that HERA is able to investigate proton structure over transverse distances ranging from the proton radius, $r_{p} \approx 0.8 \mathrm{fm}$, all the way down to $d \sim \frac{0.2 \mathrm{GeV} \mathrm{fm}}{\sqrt{Q^{2} \mathrm{GeV}^{2}}} \sim 10^{-3} \mathrm{fm}$, a little over one thousandth of the radius of the proton.

In the one photon exchange approximation, the cross section for electron-proton scattering can be written

$$
\frac{d^{2} \sigma}{d x d Q^{2}}=\frac{2 \pi \alpha^{2}}{x Q^{4}} Y_{+}\left(F_{2}^{p}\left(x, Q^{2}\right)-(1-\epsilon) F_{L}^{p}\left(x, Q^{2}\right)\right),
$$

where $Y_{+}=1+(1-y)^{2}$ and $\epsilon=\frac{2(1-y)}{Y_{+}}$is the polarisation of the exchanged virtual photon. At small $y$, transversely polarised virtual photons dominate while longitudinal photons take over at large $y$. The contribution to the cross section of the structure function $F_{2}^{p}\left(x, Q^{2}\right)$ is independent of the photon's polarisation, whereas the longitudinal structure function $F_{L}^{p}\left(x, Q^{2}\right)$ only contributes at high $y$, where the exchanged photon has polarisation $\epsilon=0$. These structure functions give access to different aspects of proton structure:

- $F_{2}^{p}\left(x, Q^{2}\right)=x \Sigma_{q} Q_{q}^{2}\left(q\left(x, Q^{2}\right)+\bar{q}\left(x, Q^{2}\right)\right)$, where the sum runs over the quark flavours, the fractional quark charges are given by $Q_{q}$ and the probability of finding a quark or anti-quark of flavour $q$ carrying a proportion $x$ of the proton's momentum at a scale $Q^{2}$ is given by $q\left(x, Q^{2}\right)$ or $\bar{q}\left(x, Q^{2}\right)$, respectively.

- $F_{L}^{p}\left(x, Q^{2}\right) \sim \alpha_{s}\left(Q^{2}\right) x g\left(x, Q^{2}\right)$, where $g\left(x, Q^{2}\right)$ is the probability of finding a gluon carrying a proportion $x$ of the proton's momentum at a scale $Q^{2}$.

Measurements of $F_{2}^{p}\left(x, Q^{2}\right)$ and $F_{L}^{p}\left(x, Q^{2}\right)$ therefore make possible the direct determination of the proportion of the proton's momentum that is carried by quarks and gluons, respectively.

One of the discoveries made by the H1 and ZEUS experiments in DIS at HERA was the dramatic increase of $F_{2}^{p}\left(x, Q^{2}\right)$ towards low $x . F_{2}^{p}\left(x, Q^{2}\right)$ is seen to behave like $x^{-\lambda}$ with $\lambda$ increasing from about 0.2 to 0.3 as $Q^{2}$ rises from 10 to $100 \mathrm{GeV}^{2}$. We note that the rise of $F_{2}^{p}\left(x, Q^{2}\right)$ must be tamed at some point. The requirement that the scattering matrix be unitary implies that ultimately $F_{2}^{p}\left(x, Q^{2}\right)$ cannot grow faster than $\ln ^{3} x$. From the measurements of $F_{2}^{p}\left(x, Q^{2}\right)$, it was deduced that the number of quarks and hence also gluons in the proton rises sharply as $x$ decreases. The extraction of the gluon density in the proton, the quantity $x g\left(x, Q^{2}\right)$ is the number of gluons per unit rapidity, relies on the assumption that the so-called "sea" quarks at low $x$, which contribute to $F_{2}^{p}\left(x, Q^{2}\right)$, are produced via gluon splitting, $g \rightarrow q \bar{q}$. Measurement of $F_{L}^{p}$ in the DIS region, $Q^{2}>5 \mathrm{GeV}^{2}$, and hence a more direct extraction of the gluon density, requires extended data taking at lower proton beam energies, foreseen for the current HERA II running period.

Unfortunately, $e^{ \pm} p$ scattering data do not allow the contributions of the different quark flavours to proton structure to be precisely identified. This requires information on the structure of the neutron that can only be obtained in the required kinematic region via the study of 
electron-deuteron $(e d)$ scattering at HERA, as is described below. The resulting information on the distributions of the different quark flavours in the proton is of interest to future experiments, for example the cross section for ultra-high-energy neutrino-nucleon interactions is dependent on the assumptions made on the low $x$ composition of the nucleons, but more importantly it is interesting in its own right. Perturbative QCD suggests that the distributions of $d$ and $u$ sea quarks, labelled $\bar{d}\left(x, Q^{2}\right)$ and $\bar{u}\left(x, Q^{2}\right)$ respectively, are the same; an expectation that is not borne out by experiment. This may indicate the influence of non-perturbative effects on proton structure, perhaps associated with the breaking of chiral symmetry in QCD.

A further discovery of the ZEUS and H1 experiments in DIS was the surprisingly large number of "rapidity gap" events in which the proton remains intact or dissociates into a system of small mass, $M_{X}<1.6 \mathrm{GeV}$. These interactions, which can be thought of as occurring with a colourless component of the proton, offer the possibility of investigating the structure of diffraction, the process responsible for the vast majority of hadron-hadron collisions. Measurements in $e^{ \pm} p$ scattering of the diffractive structure function $F_{2}^{D(3)}$, analogous to $F_{2}^{p}$, suggest that this structure is dominated by gluons, with the majority of the momentum exchanged in diffraction being carried by one gluon. Yet the process must involve (at least) two gluons, as no colour is exchanged. Investigating rapidity gap events in ed scattering will allow comparison of the structure of diffraction involving protons with that involving neutrons and also their coherent superposition, when the scattering involves the deuteron as a whole. If this structure were to prove the same in all cases it would raise some thought-provoking questions. How, for example, can we reconcile our picture of partons confined within nucleons with diffractive interactions involving gluons associated with both the nucleons in the deuteron? As is described below, further insight into these processes is provided by comparison of the total cross section for ed scattering with the sum of the $e p$ and $e n$ cross sections. Studies of the hadronic final state will also aid the development of our understanding of these coherent effects.

Experimentally, HERA offers unique possibilities for the study of ed scattering. The high centre-of-mass energy allows charged current interactions to be studied, in which a $W$ boson is exchanged. This makes possible the disentangling of the contributions of the various quark flavours to nucleon structure at high $x$. The HERA colliding beam geometry makes possible the detection of the "spectator" nucleon in ed interactions. For example, if the underlying interaction is $e n \rightarrow e X$, the spectator proton from the deuteron continues down the beam pipe with approximately half the energy of the beam deuterons and can be measured in an appropriate downstream proton tagger. The colliding beams also allow detailed studies of the hadronic final state; the detection of rapidity gaps will be as important a tool in the study of ed scattering as it was in the discovery of hard diffraction in DIS during the first phase of HERA running, HERA I.

\subsection{Structure Functions and Parton Distributions}

The $e p$ collider HERA has extended the kinematic range explored with lepton-nucleon scattering experiments by two orders of magnitude towards low Bjorken $x$ and high momentum transfers squared, $Q^{2}$. The neutral and charged current ep scattering data at high $Q^{2}$ have also provided information on parton distributions at large $x$. Despite the increasing precision of these 
measurements, the availability of lepton-proton data only does not allow the parton flavour content of the nucleon's structure in the newly opened kinematic range to be completely resolved. This, however, can be accomplished by an $e^{ \pm} D$ scattering programme which provides access to the neutron structure in the newly accessed range.

\subsubsection{Flavour asymmetry of sea quarks at low $x$}

In perturbative QCD, the sea is expected to be flavour symmetric. This prediction was first found to be violated in a measurement by the NMC Collaboration [2] of the difference $F_{2}^{p}\left(x, Q^{2}\right)-$ $F_{2}^{n}\left(x, Q^{2}\right)$ for $0.004<x<0.08$ which implied a violation of the Gottfried sum rule [3], i.e. a significant positive difference of the sea quark distributions $\bar{d}$ and $\bar{u}$. This surprising result was confirmed in Drell-Yan experiments, initially by NA51 [4] at $x=0.18$ and extended to the wider range of $0.02<x<0.345$ by the E866/NuSea Collaboration [5]. The asymmetry of the sea quarks at low $x$ has yet to be measured.

The observation of a non-vanishing $\bar{d}-\bar{u}$ difference has been intensively discussed ${ }^{1}$ in terms of Pauli exclusion at the quark level [7], of parton saturation [8], of distortions in the negative energy Dirac sea related to quark confinement [9] and of meson (pion) cloud models [10] in which the dominance of the $\pi^{+} n$ Fock component over the $\pi^{-} \Delta^{++}$state in the proton suggests that the difference $\bar{d}-\bar{u}$ is positive.

HERA provides the unique possibility to measure the sea quark asymmetry down to very low $x$ for a wide range of $Q^{2}$. This will pin down the flavour composition of the partons responsible for the rise of $F_{2}^{p}\left(x, Q^{2}\right)$ towards low $x$, allowing full pQCD based unfolding of the parton distributions. So far all NLO QCD analyses assume that $\bar{d}-\bar{u}$ tends to zero at low $x$, that is they set $A_{u}=A_{d}$ and $B_{u}=B_{d}$ in the low $x$ parton parameterisation $q(x) \propto A_{q} \cdot x^{B_{q}}$ at some initial scale $Q_{0}^{2}$. As is illustrated in figure 3, these assumptions lead to an artificially narrow uncertainty band for the parton distributions determined in the fitting procedure [11]. In an unconstrained fit, the uncertainties become large at low $x$, becoming as large as $50 \%$ for the down quark distribution at $x \simeq 10^{-4}$. The uncertainty of the resulting asymmetry $\bar{d}-\bar{u}$ increases dramatically towards low $x$, as is demonstrated in fig.4.

The measurement of the $\bar{d}-\bar{u}$ asymmetry at low $x$ is of importance for various aspects of LHC physics, including the measurement of the luminosity. Since luminosity determination based on reactions such as elastic $p p$ scattering is limited to about $5 \%$ accuracy, challenging proposals are being pursued to determine the luminosity more precisely by measuring the pseudorapidity distribution of leptons resulting from the decay of $Z$ and $W^{ \pm}$bosons produced in reactions such as $u \bar{d} \rightarrow W^{+}$at the LHC [12]. Understanding the measured rates from $W^{ \pm}$and $Z$ decays, i.e. the full set of quark and gluon contributions to higher orders in pQCD, will be much facilitated by accurately disentangling the sea quark distributions at low $x$ at HERA ${ }^{2}$. Note that boson production in $p p$ scattering depends on processes such as $c \bar{s} \rightarrow W^{+}$and $\bar{c} s \rightarrow W^{-}$ as well as gluon initiated processes. Generally, $W$ production from sea quarks is a bench-mark process [13] of high cross section at $p p$ colliders, and the determination of the low $x$ sea quark

\footnotetext{
${ }^{1}$ Considerable interest exists in the flavour asymmetry in bound nuclei as compared to the free nucleon which requires $e A$ collider data for a pair of mirror nuclei with proton and neutron enriched structure, respectively [6].

${ }^{2}$ The "partonic" luminosity measurement at the LHC covers a wide range of low values of Bjorken $x$, determined by $x_{u} \times x_{\bar{d}}=M_{W}^{2} / s=3.3 \times 10^{-5}$ for $7 \mathrm{TeV}$ beam energy.
} 

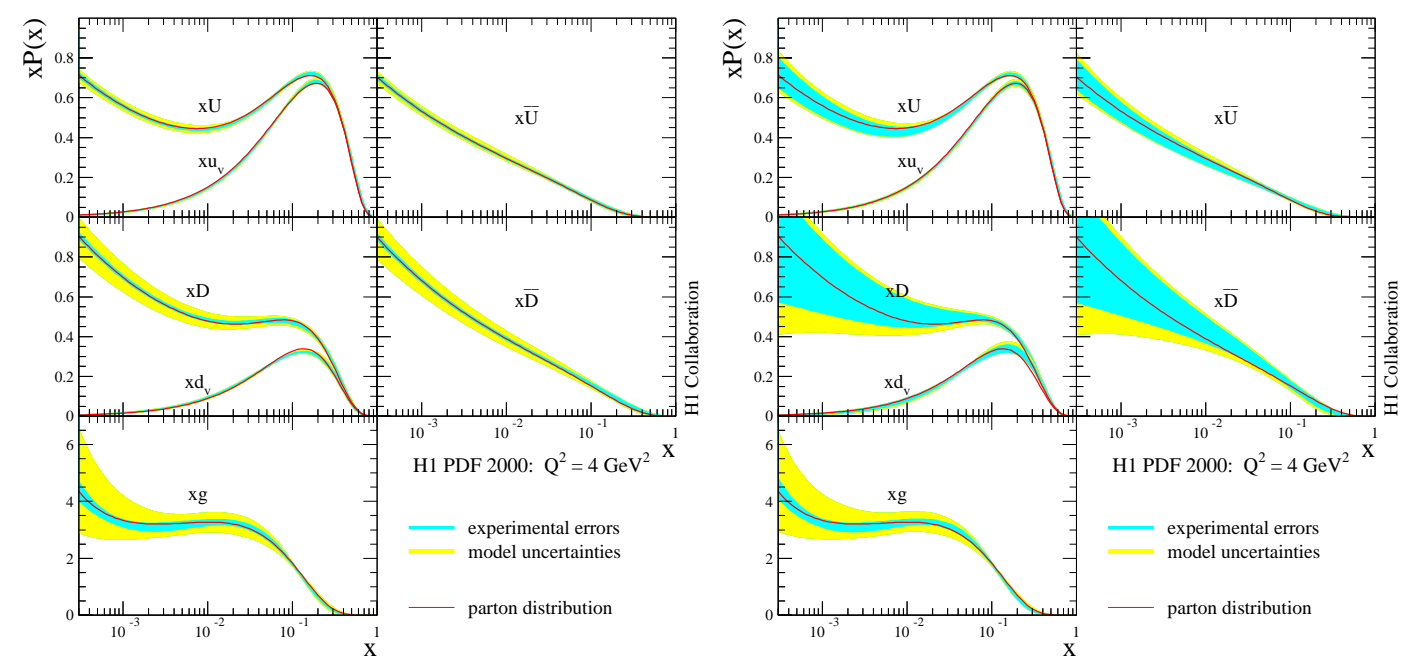

Figure 3: Parton distributions, at $Q_{0}^{2}=4 \mathrm{GeV}^{2}$, determined in a NLO QCD analysis by $\mathrm{H} 1$ of their $e p$ data and $\mu p$ and $\mu D$ data of BCDMS. The left curves represent the initial total $u$, total $d$, total $\bar{u}$ and total $\bar{d}$ quark distributions and the gluon distribution, obtained when the constraint $\bar{u}=\bar{d}$ for $x \rightarrow 0$ is imposed. The bands represent the uncertainty arising from experimental errors and the assumptions made in the analysis, see [11]. Allowing the normalisation coefficients $A_{q}$ and the $x$-dependence, i.e. the parameter $B_{q}$, to be different for $\bar{u}$ and $\bar{d}$ leads to a much wider uncertainty band, representing the genuine uncertainty of the sea quark distributions at low $x$.
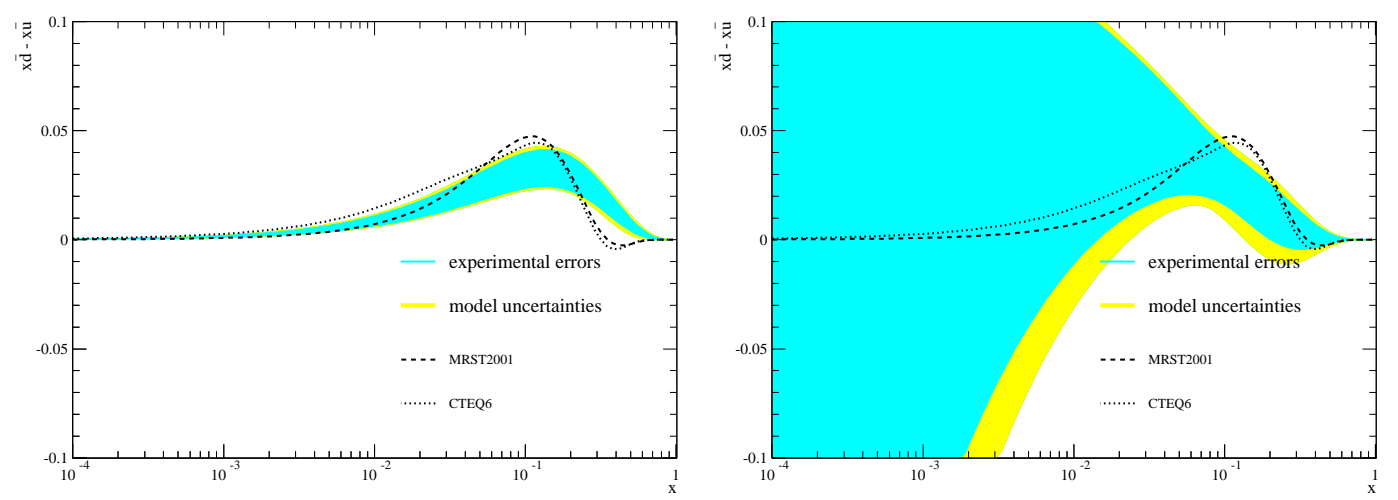

Figure 4: The sea quark distribution difference $\bar{d}-\bar{u}$, at $Q_{0}^{2}=4 \mathrm{GeV}^{2}$, as determined in a NLO QCD analysis of the H1 ep and BCDMS $\mu p$ and $\mu D$ data. The bands represent the uncertainty arising from the experimental errors and the assumptions made in the analysis, see [11]. Left: the constraint $\bar{u}=\bar{d}$ for $x \rightarrow 0$ is imposed. The enhancement at large $x$ for the global fits is largely determined by the Drell-Yan data and well reproduced in the particular H1 and BCDMS fit. Right: The normalisation coefficients $A_{q}$ and the $x$-dependence, i.e. $B_{q}$, of $\bar{u}$ and $\bar{d}$ are free parameters in the fit. This leads to a much wider uncertainty band extending to +0.3 and -0.4 at $x=10^{-4}$. 
distributions is thus essential for monitoring new particle production at the LHC. Without ed scattered at HERA the partonic structure of the nucleon cannot be mapped completely in DIS.

Further interest in the behaviour of the sea quarks at low $x$ arises from superhigh energy neutrino physics which is being pursued at various neutrino telescopes [14], including the experiment AMANDA at the South Pole. Due to their feeble interactions, neutrinos traverse the universe almost without attenuation and deflection, therefore pointing back to their sources. They thus offer a unique possibility to investigate astrophysical objects at large distances and the as yet mysterious mechanisms of hadron acceleration to the highest energies. In addition, highest-energy neutrinos are expected to probe our understanding of the transport of cosmic rays through the universe (GZK neutrinos).

For the interpretation of the data of the neutrino telescopes, as well as for the optimisation of their design, a detailed and precise knowledge of the neutrino cross sections with nucleons and nuclei and of the respective parton distribution functions (PDFs) is essential, as can be demonstrated by the following three examples:

- The measured neutrino flux (diffuse or from a specific source) inferred from the observed event rates is inversely proportional to the neutrino cross section.

- The relationship between the measured energies of the secondary leptons and the initial neutrino energies depends on the relationship between the up- and down-type quark and anti-quark distributions in nucleons and nuclei.

- Neutrinos with energies above $\mathcal{O}(100 \mathrm{TeV})$ are absorbed in the Earth with a non-negligible probability. The interpretation of the measured angular distribution thus requires the calculation of the "survival probability" of a neutrino as a function of its energy and its path length through the Earth, which in turn depends on the neutrino cross sections and hence the PDFs.

Currently, highest-energy neutrino cross sections are calculated on the basis of the Standard Model and standard sets of PDFs extrapolated to unexplored kinematic regions using perturbative QCD and heuristic assumptions about their functional form. Higher energies involve lower values of Bjorken- $x$, higher values of $Q^{2}$ and the contributions of third-generation quark distributions that are negligible at HERA. The uncertainties induced by the required extrapolations, in particular to lower $x$, are generally thought to be of the order of a factor of $2 \ldots 10$ at the highest neutrino energies, although some authors estimate that these uncertainties are of the order of $20 \%[15,16]$. As has been emphasised in [16], the accurate knowledge of the parton distributions is essential for possible discoveries, such as observing the effects of extra dimensions, since neutrino astrophysics accesses energies far beyond the energy scale of current accelerators ${ }^{3}$.

We also note that the unpolarised sea quark asymmetry provides important information on the polarised sea quark distributions [18].

\footnotetext{
${ }^{3}$ HERA data from $e A$ scattering will provide new information on low $x$ parton behaviour as the parton density in nuclei is high, effectively allowing lower $x$ to be probed than with $e p$ at a given centre-of-mass energy, see section 3.5. This is of particular interest with regard to the question of the saturation of parton densities at values of $x$ relevant to neutrino scattering at $E_{\nu} \simeq 10^{8} \mathrm{GeV}$. Such saturation must occur at some $x$ value due to unitarity considerations, see e.g. [17].
} 

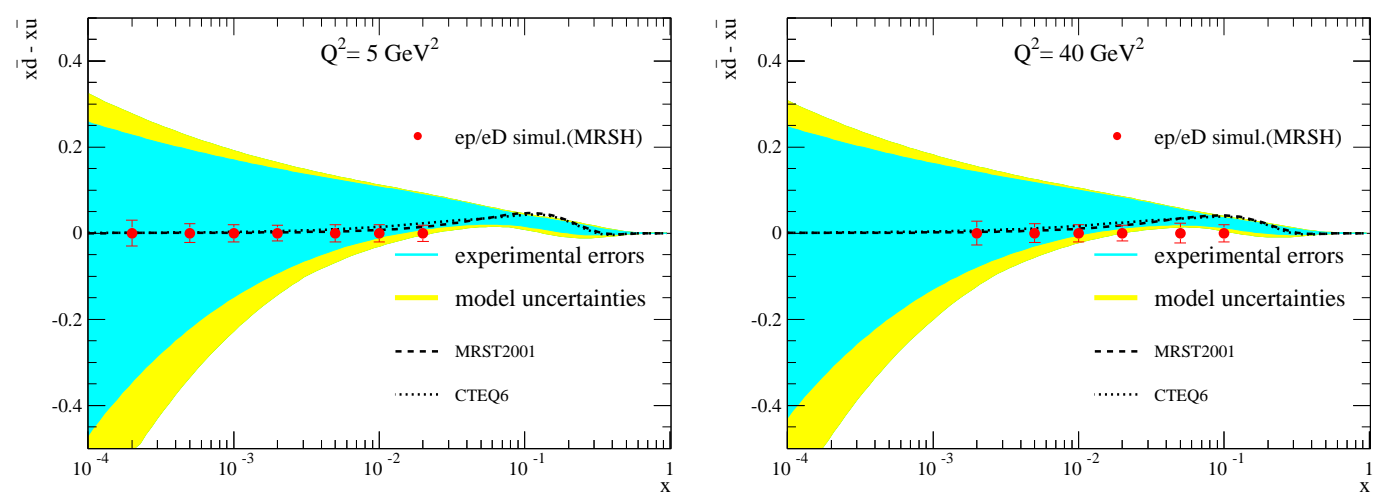

Figure 5: Simulation of the difference $\bar{d}-\bar{u}$, here assumed to be zero at low $x$, using $40 \mathrm{pb}^{-1}$ of $e p$ and $20 \mathrm{pb}^{-1}$ of $e d$ data to determine $F_{2}^{p}$ and $F_{2}^{N}$ which allows the flavour symmetry at low $x$ to be tested. The error bars represent the quadratic sum of the statistical and systematic errors, excluding that arising from the luminosity measurements of about $1 \%$. The bands represent the uncertainty of the H1 NLO QCD fit, see [11], without the constraint that $\bar{d}-\bar{u}=0$ at low $x$.

A precise measurement of the flavour asymmetry can be made from the difference of the proton and the nucleon structure functions which, for 4 flavours, are given by

$$
F_{2}^{p}=\frac{x}{9}\left[4 u_{v}+d_{v}+8 \bar{u}+2 \bar{d}+8 c+2 s\right]
$$

and

$$
F_{2}^{N}=\frac{1}{2}\left(F_{2}^{p}+F_{2}^{n}\right)=\frac{x}{9}\left[5\left(u_{v}+d_{v}\right) / 2+5 \bar{u}+5 \bar{d}+8 c+2 s\right]=\frac{5}{18} x \Sigma-\frac{1}{3} x(s-c),
$$

where $u_{v}$ and $d_{v}$ are the valence quark distributions and we are assuming that $u_{s}=\bar{u}, d_{s}=\bar{d}$, $c=\bar{c}$ and $s=\bar{s}$. Here, $\Sigma$ is the singlet combination of parton distributions, $\Sigma=\sum q+\bar{q}$, and $2 F_{2}^{N}=F_{2}^{d}$, the structure function of the deuteron ${ }^{4}$. The nucleon and proton structure function difference at low $x$, where the valence quark distributions are negligible, yields

$$
F_{2}^{N}-F_{2}^{p}=\frac{x}{3}(\bar{d}-\bar{u})
$$

A simulation [19] of this measurement using the present $\mathrm{H} 1$ apparatus and systematic uncertainties, as achieved in the low $Q^{2} \mathrm{H} 1$ precision $F_{2}$ data [20], is shown in figure 5. Corrections for the effects of shadowing can be applied with the necessary precision (see section 2.3 and $[21,22]$ ). The accuracy of this measurement is estimated to be about 0.03 which is an order of magnitude more precise than the uncertainty obtained with the current data in an unconstrained fit.

The exploration of the low $x$ behaviour of the neutron structure function and its $Q^{2}$ dependence requires a luminosity of $\sim 20 \mathrm{pb}^{-1}$.

\footnotetext{
${ }^{4}$ We reserve the expression $F_{2}^{d}$ for the structure function of the deuteron and use the symbols $F_{2}^{D(4)}$ and $F_{2}^{D(3)}$ to refer to the diffractive structure functions of the nucleon, distinguishing between nucleons with an additional subscript where necessary.
} 


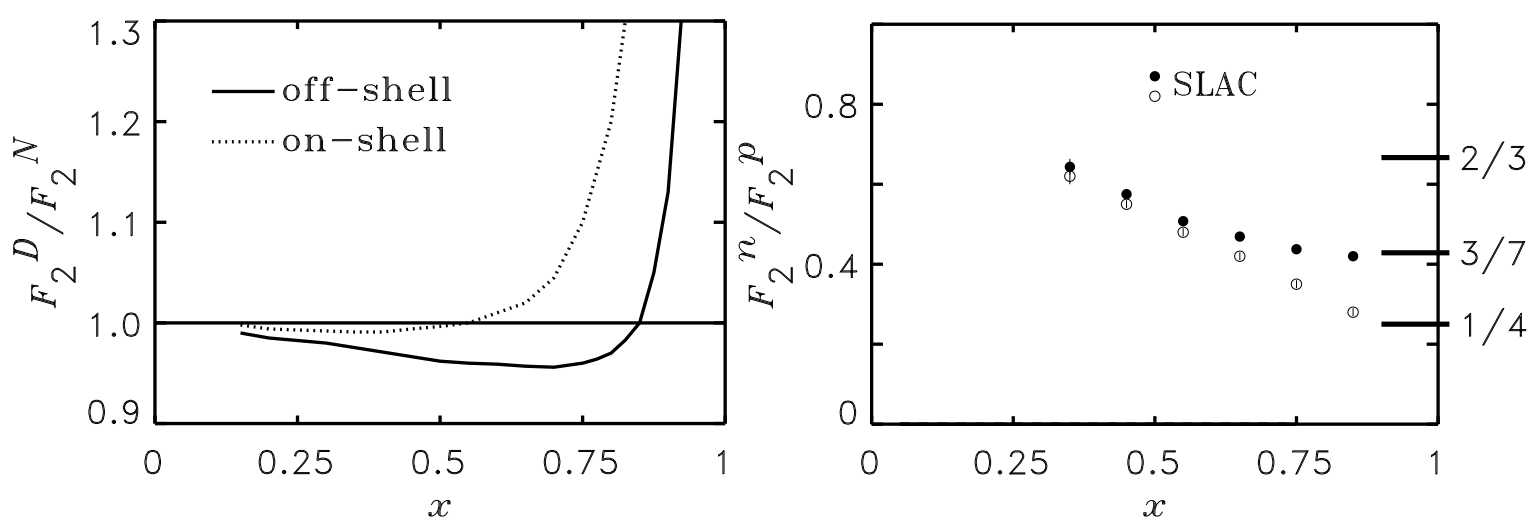

Figure 6: Ratio of the deuteron to the nucleon structure functions calculated in the relativistic off-shell model (solid) and on-shell model (dashed) including Fermi motion (left). Neutron to proton structure function ratio for the SLAC $e N$ DIS [27] data using the off-shell and the on-shell model of the deuteron (right). Both figures are taken from [28].

\subsubsection{Valence quarks at large $x$}

A further reason for the interest in $F_{2}^{n}$ is the possibility it offers to disentangle the valence quark distributions at large $x$. The recent observations of large cross sections at high $E_{t}$ at FNAL illustrate the importance of accurate knowledge of the $u$ and $d$ quark distributions at high $x$ in the search for new phenomena. Moreover, measurements of the Drell-Yan cross sections in $p p$ and $p D$ collisions [23] by the E866/NuSea Collaboration suggest that the valence quark distributions at high $x$ may be overestimated in the global fit analyses. Recently, a finite limit of 0.2 for $d_{v} / u_{v}$ at $x=1$ has been introduced in fits [24], instead of the conventional assumption that this ratio approaches zero, see also [25].

In the high $x$ region, the ratio of the proton to neutron structure functions is bound by $1 / 4$ and 4 according to the relation

$$
\frac{F_{2}^{n}}{F_{2}^{p}} \rightarrow \frac{1+4 d_{v} / u_{v}}{4+d_{v} / u_{v}}
$$

In naive $\mathrm{SU}(6)$, this ratio approaches $2 / 3$ since $d / u=1 / 2$, while it is $1 / 4$ if scalar diquark dominance is assumed, i.e. if $d / u=0$. Light nuclei, in particular deuterium, can and have been used to determine the neutron structure function $F_{2}^{n}\left(x, Q^{2}\right)$ at high $x$. At the highest values measured by BCDMS, $x=0.75$, the ratio $\frac{F_{2}^{n}}{F_{2}^{p}}$ was observed to be $0.326 \pm 0.028$ (stat.) at $Q^{2}=60 \mathrm{GeV}^{2}[26]$.

A major complication in such measurements is the sizeable corrections that must be applied for nuclear binding effects. Depending on the model assumptions, rather large differences arise in the ratio of the proton and neutron structure functions which, as is illustrated in figures 6 and 7 , may exceed the difference between the expectations described above.

The measurement of the $d / u$ ratio at $\mathrm{H} 1$ using deuteron scattering can profit from two important advantages over previous fixed target experiments: tagging of the spectator proton and reconstruction of its momentum to an estimated accuracy of $1 \%$, see section 5.2. The latter point 


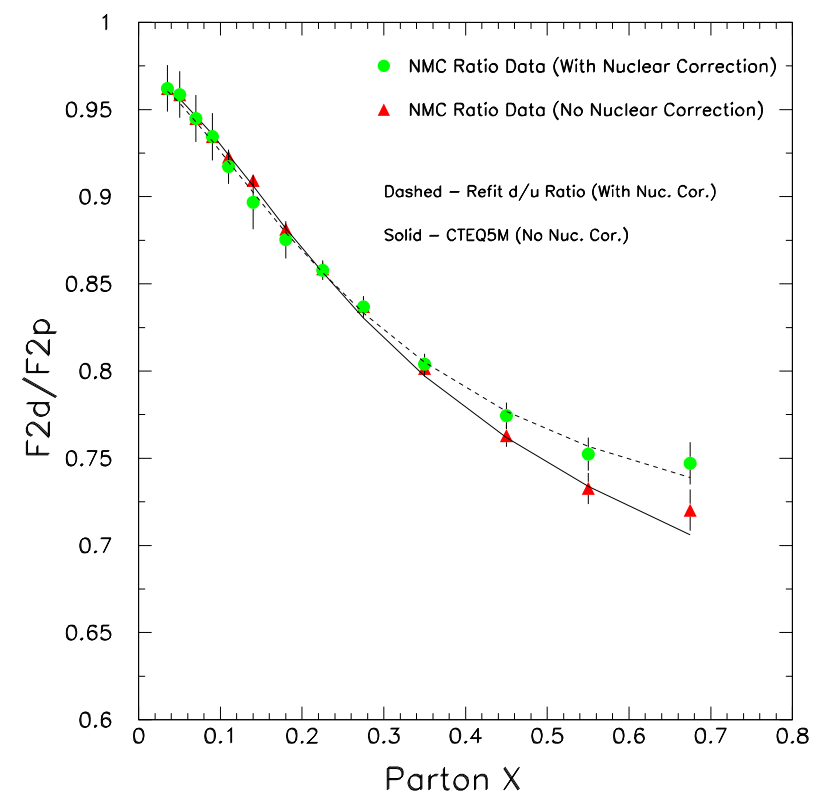

Figure 7: Effect of nuclear corrections on the structure function ratio $F_{2}^{d} / F_{2}^{p}$ from the NMC data as studied by the CTEQ collaboration [25].

allows reconstruction of $e n$ scattering with much reduced Fermi motion corrections ${ }^{5}$. Moreover, radiative corrections, which are known to be sizeable at high $x$, can be much reduced by requiring momentum balance as is possible with the HERA detectors. Because the cross section drops rapidly (with $(1-x)^{3}$ ) towards high $x$, luminosities $\mathcal{O}\left(50 \mathrm{pb}^{-1}\right)$ will be required to access this region. Data need to be obtained in a proton run with a beam energy of half of the nominal value, and in $e d$ mode in order to ensure at least partial cancellation of the systematic errors. The simulated result of such a measurement is illustrated in figure 8 . The expected systematic errors need further evaluation. It is clear that measuring the steep decrease of the cross section towards large $x$ is a challenge for the unfolding procedure and the detector resolution, both of which require further study. These factors will determine the maximum $x$ that can be measured.

In $e p$ scattering a complementary determination of the behaviour of the valence quark distributions at large $x$ can be obtained from high luminosity charged current cross section measurements, on $d_{v}$ from positron-proton scattering and on $u_{v}$ from electron-proton scattering. More accurate data on $2 u_{v}+d_{v}$ will become available from the interference structure function $x F_{3}$. These measurements are free of nuclear corrections but, even for very large luminosity, the access to the high $x$ region will be statistically limited because of smallness of the weak charged and neutral current cross sections and the unfavourable $y$ dependence.

\subsubsection{Parton distributions and measurement of the strong coupling constant}

The strong coupling is the least well measured of the fundamental constants of nature, see table 1 , despite the efforts of many experiments and the increasing precision and complexity

\footnotetext{
${ }^{5}$ Recently an experiment was endorsed at JLAB which aims to measure "The Structure of the Free Neutron via Spectator Tagging" for $Q^{2}$ from 1 to $5 \mathrm{GeV}^{2}$ and $W$ near the resonance region [29].
} 
$2003 / 04 / 03 \quad 19.26$

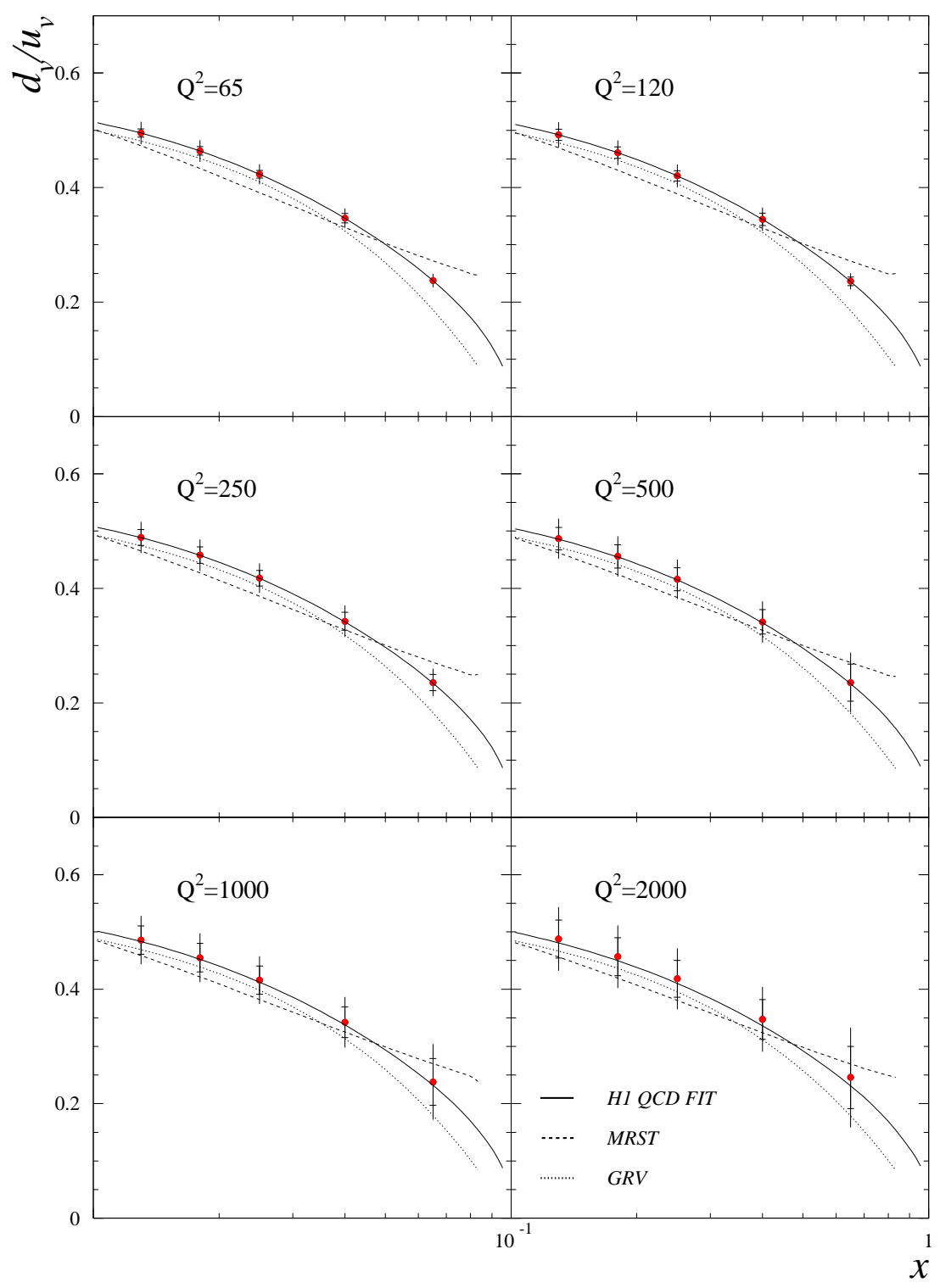

Figure 8: Simulation of the measurement of the ratio $d_{v} / u_{v}$ with $\mathrm{H} 1$ based on $50 \mathrm{pb}^{-1}$ luminosity for $e p$ and for $e d$ scattering. 


\begin{tabular}{|l|c|c|}
\hline Coupling constant or mass & Value & Relative experimental error \\
\hline Fine structure constant $\alpha$ & $1 / 137.03599976(50)$ & $3.7 \times 10^{-9}$ \\
Fermi constant $G_{F}$ & $1.16639(1) \mathrm{GeV}^{-2}$ & $8.6 \times 10^{-6}$ \\
$Z$ boson mass & $91.1876(21) \mathrm{GeV}$ & $2.3 \times 10^{-5}$ \\
$W$ boson mass & $80.423(39) \mathrm{GeV}$ & $4.8 \times 10^{-4}$ \\
Gravitational constant $G_{N}$ & $6.673(10) 10^{-11} \mathrm{~m}^{3} \mathrm{~kg}^{-1} \mathrm{~s}^{-2}$ & $1.5 \times 10^{-3}$ \\
Strong coupling constant $\alpha_{s}\left(M_{Z}\right)$ & $0.1172(20)$ & $1.7 \times 10^{-2}$ \\
\hline
\end{tabular}

Table 1: Coupling constants and gauge boson masses characterising the $\mathrm{SU}(3) \times \mathrm{SU}(2)_{L} \times \mathrm{U}(1)$ standard model of parton interactions and gravitation from [1]. Continued efforts are needed to improve the accuracy of measurements of $\alpha_{s}$.

of theoretical calculations [34]. The behaviour of the coupling constants [35], and even their possible time variation in a world with extra dimensions, is the subject of active discussions [36].

A recent H1 measurement of $\alpha_{s}$, based on H1 and BCDMS DIS data [20], provides one of the most precise determinations of this coupling, with an experimental precision of $1.5 \times 10^{-2}$, see also [37]. An analysis of all available DIS data has determined $\alpha_{s}$ to NNLO using all available moments with an accuracy and value close to that published in the Particle Data Book, see [35]. As is well known, the use of muon fixed target data in the determination of the strong coupling constant is problematic. The BCDMS data force $\alpha_{s}$ to be very small $(\simeq 0.110)$ while the NMC data require that higher twist corrections be made in order to weaken the otherwise strong dependence of $\alpha_{s}$ on the minimum $Q^{2}$ used [38,39]. This suggests that data should be taken in an extended period of low energy running at HERA with $E_{p}=460 \mathrm{GeV}$, as is also advisable for the $920 \mathrm{GeV}$ ed programme, in order to re-measure the high $x$ region at moderate values of $Q^{2}$ with the collider detectors. These have the advantage of measuring the kinematics with both the scattered electron and the hadronic final state, thereby reducing the systematic errors and radiative corrections considerably. The possibility of suppressing nuclear corrections by the tagging of spectator nucleons means the HERA ed data will be of higher quality than hitherto in such analyses.

In order to determine $\alpha_{s}$ to better than the world average accuracy, the HERA inclusive cross section measurements need to be improved in accuracy to below the per cent level, i.e. by a factor of 3 . This is possible for the $\mathrm{H} 1$ apparatus with high statistics by using redundant tracking and high resolution calorimetry [40]. The rôle of deuteron data in this programme was sketched back in 1996 in an analysis of simulated data prior to the recent H1 QCD studies [41]. They constrain the non-singlet parton distributions and thus lead to improved stability of the fit results and determinations of non-singlet structure functions at small $x$. The evolution of the difference $F_{2}^{p}-F_{2}^{n}$ is independent of the gluon distribution and thus provides an interesting determination of $\alpha_{s}$ which is free of the dependence on the shape of $x g$ which is problematic in $F_{2}^{p}$ analyses [39]. The measurement of this difference with the $\mathrm{H} 1$ detector can be performed in a wide region of $x$ and $Q^{2}$, testing the $\ln Q^{2}$ evolution up to large $Q^{2}$ values. In order to estimate the genuine impact of the $e d$ data on a precision measurement of $\alpha_{s}$ a new, dedicated study is required using the latest fit techniques and more appropriate assumptions on the accuracy of the cross section measurement. The expectation is that the precision of the $\alpha_{s}$ measurements will 


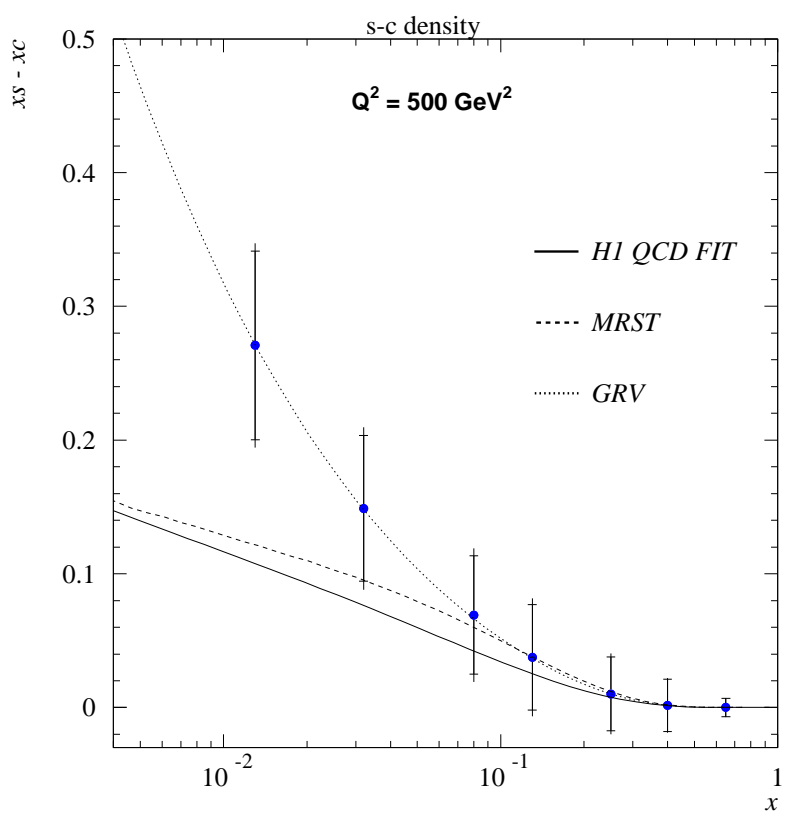

Figure 9: Simulated measurement of the difference of the strange and charm sea distributions in the nucleon from a comparison of the structure functions $F_{2}^{N}$ and $W_{2}^{N}$, determined in NC and $\mathrm{CC} e^{ \pm} d$ scattering. The estimated uncertainty, which is dominated by the statistical accuracy of $\mathrm{CC}$ data, is based on a luminosity of $100 \mathrm{pb}^{-1}$ and includes acceptance and trigger efficiency corrections.

improve and become much more stable in conjunction with the improved information on the PDFs. IN this measurement, precision data on $F_{L}$ are expected to be of crucial importance as they help to fix the initial gluon distribution.

With the precision measurements of $e^{ \pm} p$ and tagged $e^{ \pm} n \mathrm{NC}$ and $\mathrm{CC}$ cross sections, including the charm, beauty and strange measurements, the $\mathrm{H} 1$ experiment will be able to accurately and consistently determine all parton distributions: the gluon distribution $x g$; the quark distributions $\bar{u}, \bar{d}, u, d, s, c, b$ and the up and down valence distributions in a broad and hitherto inaccessible $x$ range, as well as investigating their evolution as a function of the scale.

A further example of this is the determination of the difference of the strange and charm distributions. The reduced charged current $e^{ \pm}$scattering cross section

$$
\sigma_{c c}^{ \pm}=Y_{+} W_{2}^{ \pm} \mp Y_{-} x W_{3}^{ \pm}
$$

can be simplified by using the relations

$$
\begin{array}{ll}
W_{2}^{+, N}=x\left[u_{v}+d_{v}+2(\bar{u}+\bar{d}+s+\bar{c})\right] & x W_{3}^{+, N}=x\left[\left(u_{v}+d_{v}\right)+2(s-\bar{c})\right] \\
W_{2}^{-, N}=x\left[u_{v}+d_{v}+2(\bar{u}+\bar{d}+\bar{s}+c)\right] & x W_{3}^{-, N}=x\left[\left(u_{v}+d_{v}\right)-2(\bar{s}-c)\right]
\end{array}
$$

for the charged current structure functions. For an isoscalar target, and assuming $s=\bar{s}$ and $c=\bar{c}$, there is only one CC structure function, $W_{2}^{N}=x \Sigma$, independent of the lepton beam 
charge, while $x W_{3}$ measures the sum of the valence quark distributions with a small correction $\pm(s-c)$ in $e^{ \pm}$scattering. The combination $s-c$ becomes directly accessible through the relationship

$$
s-c=\frac{5}{6} W_{2}^{N}-3 F_{2}^{N} .
$$

This measurement has been simulated, see figure 9. There are rather striking differences between the predictions of different global analyses regarding the $s-c$ distribution which may be resolved in a high statistics measurement. The determination of $s-c$ and $c$, with forward charm production at high $Q^{2}$ and $x$ is of interest for testing the hypothesis of intrinsic nucleon heavy flavour [42] and, as was noted above, for the partonic luminosity measurement at the LHC. The determination of $s$ resulting from an analysis such as that sketched here is expected to be more accurate than the complementary study of strange quark production based on the observation of charm in CC.

The programme of unfolding the parton distributions is both fascinating in its own right and essential for predicting $p p$ and neutrino scattering cross sections. Neither the first discovery phase of HERA nor the high luminosity run which are just beginning are able to complete this programme, as it requires the exploration of the partonic composition of the neutron as well as that of the proton.

\subsection{Nuclear shadowing in deuterium}

So far we have concentrated on the measurements of nucleon structure that the HERA III electron-deuteron scattering programme will make possible. However, studying ed collisions with HERA will also allow the first check to be made of the deep connection between the phenomena of high energy diffraction and nuclear shadowing, originally demonstrated by Gribov [43]. An understanding of shadowing is also necessary to enable correction of the measurements of $F_{2}^{n}$ for nuclear effects.

Nuclear shadowing in DIS on the deuteron arises from the interference between the amplitudes for the diffractive scattering of the projectile off the proton and the neutron in the deuterium target. Such interference is possible at small $x, x \leq 5 \times 10^{-2}$, where the minimum momentum transfer to the nucleon $\sim x m_{N}$, where $m_{N}$ is the nucleon mass, becomes smaller than the average nucleon momentum in the deuteron. The corresponding double scattering diagram for the $\gamma^{*} d$ scattering, whose imaginary part is, through the optical theorem, proportional to some cross section that is commonly called $\sigma_{2}$, leads to a diffractive final state and increases the probability of diffraction on the nucleus by $\sigma_{2}$ (here, we neglect small effects associated with the real part of the elementary diffractive amplitude - see below). Application of the cutting rules of Abramovsky, Gribov and Kancheli [44] demonstrates that the interference diagram decreases the total cross section for $\gamma^{*} d$ scattering, i.e. it leads to nuclear shadowing with magnitude $\sigma_{2}$. The cross section for inelastic interactions with a single nucleon is also seen to decrease by $\sigma_{\text {single }}=-4 \sigma_{2}$, while the cross section for simultaneous inelastic interactions with two nucleons becomes $\sigma_{\text {double }}=2 \sigma_{2}$. 
The Gribov theory allows us to relate $\sigma_{2}$ to the cross section for the diffractive scattering of the virtual photon in the same kinematic region. For example, for DIS on a deuterium target

$$
\begin{aligned}
F_{2}^{d}\left(x, Q^{2}\right)= & F_{2}^{p}\left(x, Q^{2}\right)+F_{2}^{n}\left(x, Q^{2}\right) \\
& -2 \frac{1-\eta^{2}}{1+\eta^{2}} \int_{x}^{x_{0}} d x_{\mathbb{P}} d q_{t}^{2} F_{2}^{D(4)}\left(\beta, Q^{2}, x_{\mathbb{P}}, t\right) \rho_{d}\left(4 q_{t}^{2}+4\left(x_{\mathbb{P}} m_{N}\right)^{2}\right),
\end{aligned}
$$

where $F_{2}^{D(4)}$ is the nucleon diffractive structure function, $\rho_{d}$ is the deuteron form factor and $|t|=$ $q_{t}^{2}+\left(x_{\mathbb{P}} m_{N}\right)^{2}$. Since the $t$-dependence of $\rho_{d}$ is rather moderate (compared to heavier nuclei), the integral in equation (9) is sensitive to $F_{2}^{D(4)}(t)$ up to $-t \leq 0.05 \mathrm{GeV}^{2}$. In equation (9), the factor $\left(1-\eta^{2}\right) /\left(1+\eta^{2}\right)$, where

$$
\eta=-\pi / 2 \partial \ln \left(\sqrt{f_{i / N}^{D}}\right) / \partial \ln \left(1 / x_{\mathbb{P}}\right)=\pi / 2\left(\alpha_{\mathbb{P}}(t=0)-1\right)
$$

accounts for the real part of the amplitude for the diffractive scattering and leads to a reduction of the shadowing by about $20 \%$ [45].

Using the QCD factorisation theorem for diffraction [46], it is possible to extend the Gribov theory to calculate the magnitude of nuclear shadowing effects for the quark and gluon parton densities of the deuteron at small $x$ (see reference [47] and subsequent publications [48,49]),

$$
\begin{aligned}
f_{j / d}\left(x, Q^{2}\right)= & f_{j / p}\left(x, Q^{2}\right)+f_{j / n}\left(x, Q^{2}\right) \\
& -2 \frac{1-\eta^{2}}{1+\eta^{2}} \int_{x}^{x_{0}} d x_{\mathbb{P}} d q_{t}^{2} f_{j / N}^{D}\left(\beta, Q^{2}, x_{\mathbb{P}}, t\right) \rho_{d}\left(4 q_{t}^{2}+4\left(x_{\mathbb{P}} m_{N}\right)^{2}\right) .
\end{aligned}
$$

The results of the calculation of the ratios $F_{2}^{d} /\left(F_{2}^{p}+F_{2}^{n}\right)$ and $g_{d} /\left(2 g_{N}\right)$ using the H1 diffractive fit [50] for $F_{2}^{D(3)}$ are presented in figures 10 and 11 for a range of $Q^{2}$ and $x$.

Substantial shadowing is expected in the small $x$ region. Note also that nuclear shadowing for $F_{2}^{d}$ for $Q^{2} \leq 1 \mathrm{GeV}^{2}$ is expected to be substantially larger than for $Q^{2} \sim 4 \mathrm{GeV}^{2}$ (up to a factor of two) due to the enhancement of diffraction at small $Q^{2}$ because of the higher twist effects, such as vector meson production.

Since the diffractive cross sections are likely to be known with an accuracy of about $10 \%$, it appears that the accuracy of the calculation of the nuclear shadowing correction for the inclusive cross section will be better than $0.5 \%$ for a wide range of $Q^{2}$. Correspondingly, the theoretical uncertainty for the ratio of $F_{2}^{n} / F_{2}^{p}$ will not exceed $1 \%$.

\subsubsection{Tagging spectator protons and neutrons}

A strategy complementary to the inclusive measurement of $F_{2}^{D}$ will be the use of the neutron and proton tagging. The two options are to tag the scattering off neutrons via detection of the leading protons, and vice versa. The suitability of the current $\mathrm{H} 1$ apparatus, and the upgrades necessary to allow this are discussed in section 5.2.

As explained above using the AGK cutting rules, the overall correction for nuclear effects in non-diffractive events is 4 times larger than for inclusive scattering. However the effects 

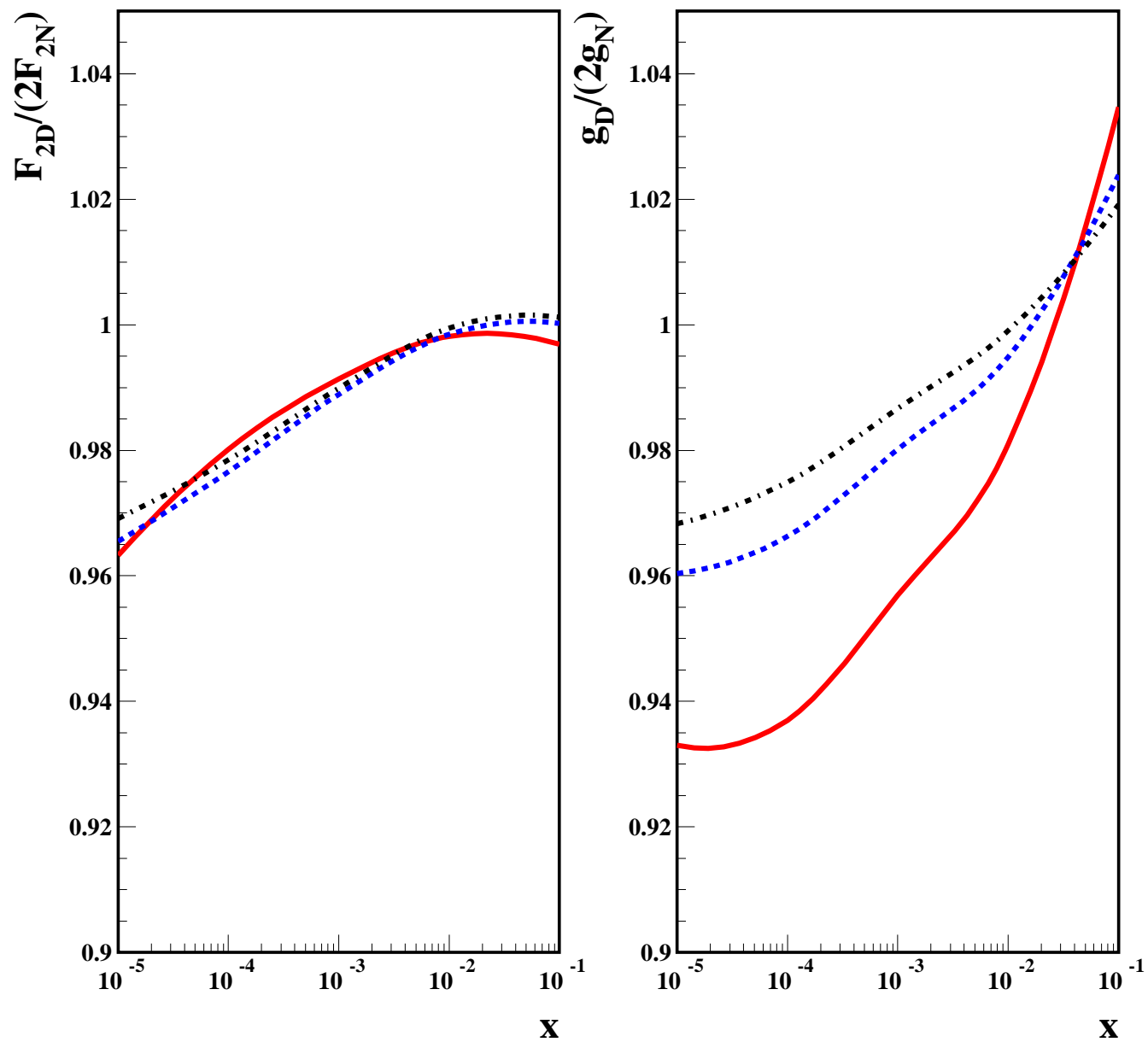

Figure 10: The ratios $F_{2}^{d} /\left(F_{2}^{p}+F_{2}^{n}\right)$ and $g_{d} /\left(2 g_{N}\right)$ as a function of $x$. The solid curve corresponds to $Q=2 \mathrm{GeV}$; the dashed curve to $Q=5 \mathrm{GeV}$ and the dash-dotted curve to $Q=10 \mathrm{GeV}$. 

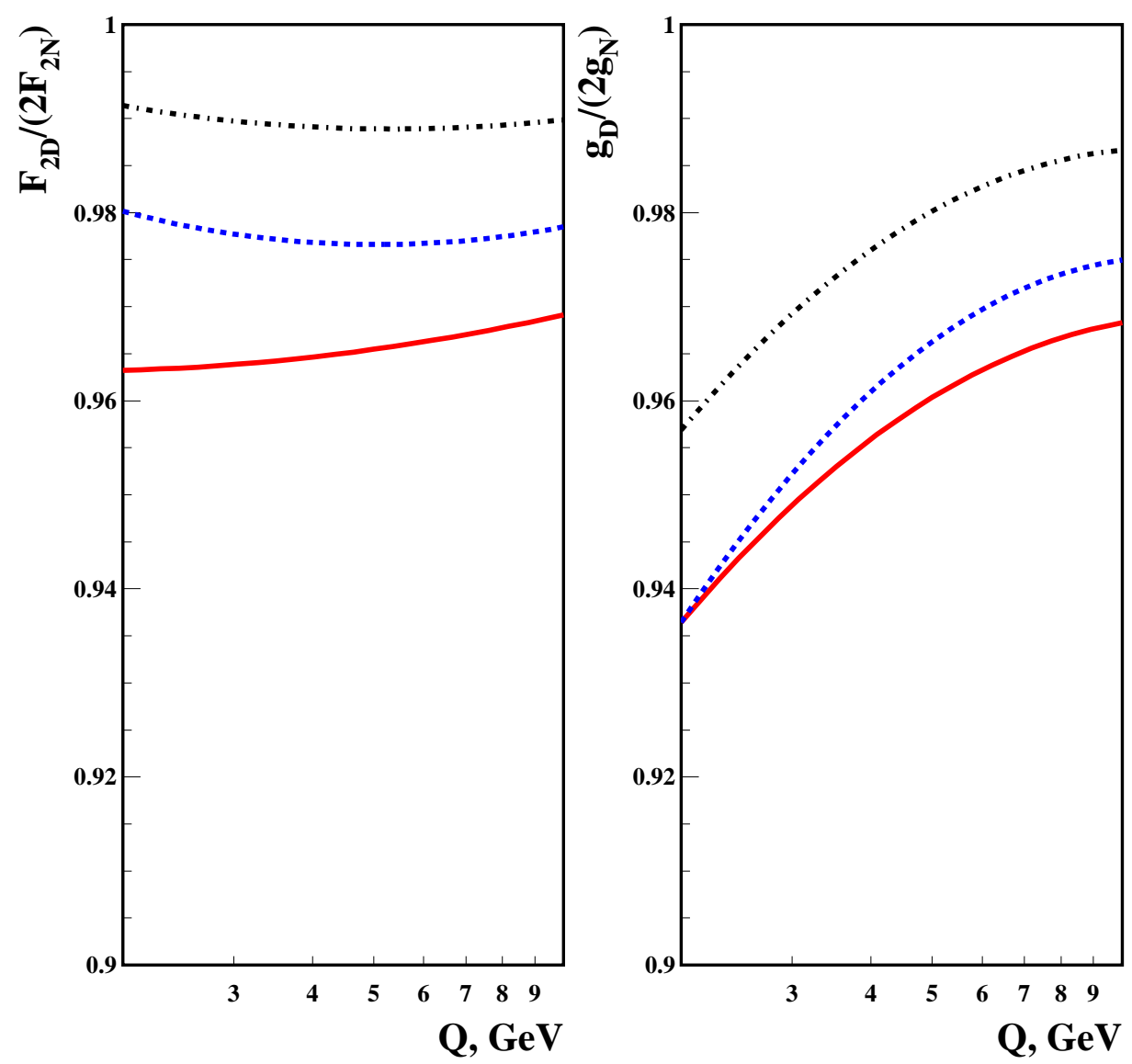

Figure 11: The ratio $F_{2}^{d} /\left(F_{2}^{p}+F_{2}^{n}\right)$ and $g_{d} /\left(2 g_{N}\right)$ as a function of $Q$. The solid curve corresponds to $x=10^{-5}$; the dashed curve to $x=10^{-4}$ and the dash-dotted curve to $x=10^{-3}$. 
are concentrated at relatively large transverse momenta $\left(k_{t}\right)$ of the protons and depend rather strongly on $k_{t}$. Hence, two strategies will be possible - one would be to select only very low $p_{t}$ protons, another is to measure the $p_{t}$ dependence of the spectrum up to $p_{t} \sim 250 \mathrm{MeV} / \mathrm{c}$ and to use this to check the theory and measure in an independent way the effects of rescattering (nuclear shadowing). Provided the momentum resolution of the proton spectrometer is adequate, it is possible to make longitudinal momentum cuts to suppress or increase the shadowing effect: these are minimal for $\left.\left|2 p_{N} / p_{d}-1\right| \leq 0.05\right)$.

The following analysis demonstrates the dependence of the nuclear shadowing correction to the $\gamma^{*} d \rightarrow p X$ cross section on the transverse momentum of the spectator proton $p_{t}$. The impulse approximation expression for the differential cross section of interest reads

$$
\left.\frac{d \sigma^{\gamma^{*} d \rightarrow p X}}{d^{3} p}\right|_{I A}=\sigma^{\gamma^{*} n}\left(2-2 x_{L}\right)\left(u^{2}(p)+w^{2}(p)\right),
$$

where $x_{L}=E_{N} / E_{D}=\left(1-p_{z} / m_{N}\right) / 2$ is the Feynman $x$ of the spectator proton, $p=\left(p_{t}, p_{z}\right)$ is the three-momentum of the detected (spectator) proton in the deuteron rest frame and $u$ and $w$ are the $S$ - and $D$-wave components of the deuteron momentum-space wave function, with the normalisation $4 \pi \int d p p^{2}\left(u(p)^{2}+w(p)^{2}\right)=1$. The presence of leading twist nuclear shadowing adds a nuclear shadowing correction to equation (12) so that the complete cross section reads

$$
\begin{aligned}
& \frac{d \sigma^{\gamma^{*} d \rightarrow p X}}{d^{3} p}=\sigma^{\gamma^{*} n}\left(2-2 x_{L}\right)\left(u^{2}(p)+w^{2}(p)\right)-\frac{3-\eta^{2}}{1+\eta^{2}} \\
& \times \int_{x}^{x_{0}} d x_{\mathbb{P}} \int \frac{d^{2} q_{t}}{\pi} F_{2}^{D(4)}\left(\beta, Q^{2}, x_{\mathbb{P}}, t\right)\left[u(p) u\left(p^{\prime}\right)+w(p) w\left(p^{\prime}\right)\left(\frac{3}{2} \frac{\left(p \cdot p^{\prime}\right)^{2}}{p^{2} p^{\prime 2}}-\frac{1}{2}\right)\right]
\end{aligned}
$$

where $p^{\prime}=p+q_{t}+\left(x_{\mathbb{P}} m_{N}\right) e_{z}$. The additional factor of three in front of the shadowing correction is a reflection of the AGK cutting rules. It is the result of a factor of 4 screening from the single inelastic sub-processes and an enhancement of the diffractive processes by a factor of $\left(1-\eta^{2}\right)$. In contrast to equation (9), we have included the integration over the direction of the vector $q_{t}$ in the transverse plane by the substitution $d q_{t}^{2} \rightarrow d^{2} q_{t} / \pi$. (We neglect here the production of deuterons in the diffractive channel which will somewhat increase the shadowing effect.)

As one can see from equation (13), nuclear shadowing suppresses the spectrum of the produced protons. This effect can be quantified by considering the ratio $R$ of the complete expression, equation (13), to the impulse approximation, equation (12). Figure 12 shows the ratio $R$ as a function of Bjorken $x$ for $p_{t}=(0,100,200) \mathrm{MeV} / \mathrm{c}$ and $p_{z}=0$. The calculation is made at $Q=2 \mathrm{GeV}$.

Two features of figure 12 are of interest and importance. Firstly, nuclear shadowing works to decrease the ratio $R$ as $p_{t}$ increases. This is as expected from our picture of nuclear shadowing since large $p_{t}$ corresponds to small transverse distances between the two nucleons generating the shadowing effect, as was first pointed out by Glauber [51]. Secondly, the suppression of $R$ at large $p_{t}$ is strikingly large. This is a common feature of semi-exclusive reactions with nuclei. Indeed, at large $p_{t}$, while the impulse approximation term is suppressed by the nuclear wave function, the rescattering term survives and gives the dominant contribution. 


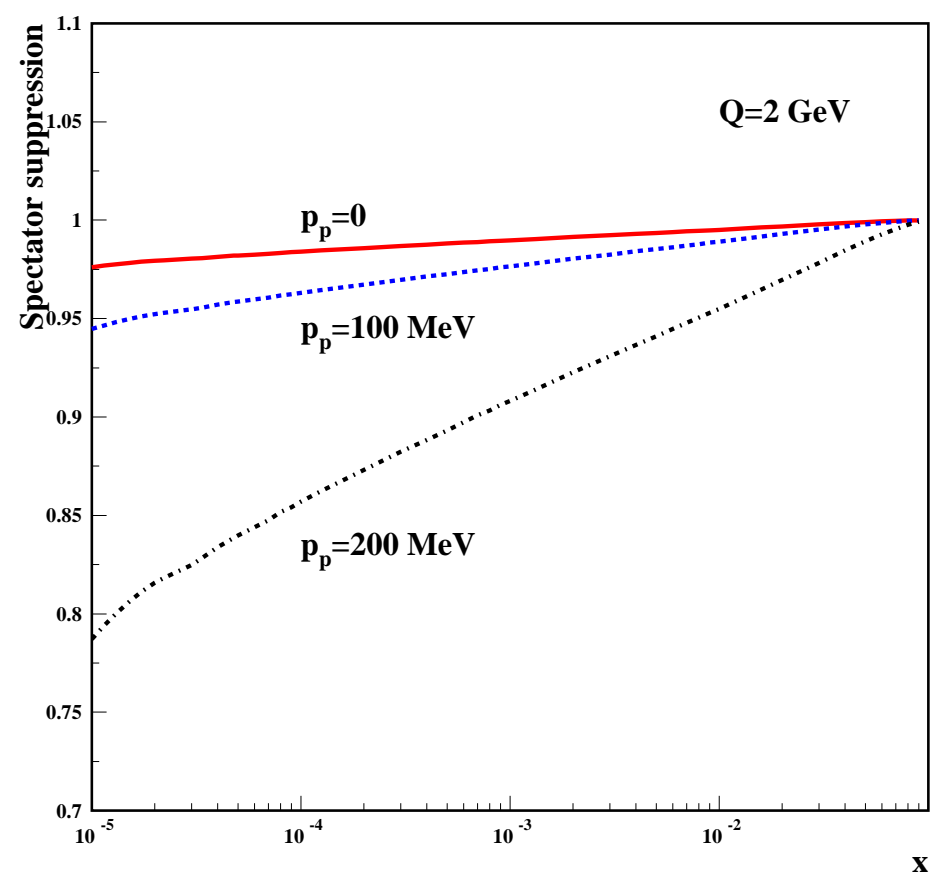

Figure 12: The suppression of the proton spectrum by the nuclear shadowing correction. The solid curve corresponds to $p_{t}=0$; the dashed curve to $p_{t}=100 \mathrm{MeV}$ and the dash-dotted curve to $p_{t}=200 \mathrm{MeV}$. 
We have also checked how the results presented in figure 12 depend on the longitudinal momentum of the spectator proton, $p_{z}$, at $\left|p_{z}\right|=100 \mathrm{MeV} / \mathrm{c}$. Taking $\left|p_{z}\right|=100 \mathrm{MeV} / \mathrm{c} \mathrm{sig}$ nificantly increases nuclear shadowing in the case with $p_{t}=0$ : at $x=10^{-5}$ and $Q=2 \mathrm{GeV}$, the ratio $R$ becomes $R=(0.93,0.94,98)$ for $p_{z}=(-100,+100,0) \mathrm{MeV} / \mathrm{c}$. For the case that $p_{t}=100$ and $200 \mathrm{MeV} / \mathrm{c}$, the effect of the increase of $p_{z}$ from zero to $100 \mathrm{MeV} / \mathrm{c}$ is much more moderate: the ratio $R$ either remains the same, for $p_{z}=-100 \mathrm{MeV} / \mathrm{c}$, or decreases by about 2 and $4 \%$, for $p_{z}=+100 \mathrm{MeV} / \mathrm{c}$.

Note also that there is a non-spectator contribution to the nucleon spectrum, which originates from diffractive scattering off the proton and which dominates at large $p_{t}, p_{t} \geq 300 \mathrm{MeV} / \mathrm{c}$. This contribution can be subtracted using the measurements at, for instance, $p_{t} \geq 400 \mathrm{MeV} / \mathrm{c}$.

\subsubsection{Simultaneous tagging of protons and neutrons}

In this case nuclear shadowing will cancel in the ratio $F_{2}^{n} / F_{2}^{p}$ and the main errors will be due to the determination of the relative efficiencies of the proton and neutron taggers.

It would also be possible to get the ratio from either the comparison of the rates of tagged proton scattering events with a neutron spectator and inclusive $e D$ scattering or of the rate of tagged neutron scattering events with the inclusive rate. Errors associated with the luminosity measurement cancel in these cases, but the measurement becomes more sensitive to the nuclear shadowing effects and errors in the acceptance of the neutron and proton taggers.

The combination of the above strategies should allow the measurement of the ratio $F_{2}^{n} / F_{2}^{p}$ with high precision. Quantification of the accuracy ultimately achievable requires detailed MC studies which are beyond the scope of this Letter of Intent.

\subsection{Diffraction}

A further area in which the ed scattering programme can lead to new insight is in the study of diffraction. The generic diffractive DIS process is illustrated in figure 13. A photon of virtuality $Q^{2}$ coupling to the electron interacts with a colourless exchange which couples to the proton. The proton remains intact, having lost a small fraction $x_{\mathbb{P}}$ of its longitudinal momentum. The squared four-momentum transferred at the proton vertex, $t$, is also typically very small. The virtual photon couples to a quark in the diffractive exchange carrying a fraction $\beta$ of the exchanged longitudinal momentum.

The development of a complete understanding of diffractive scattering processes of the type $e p \rightarrow e X p$ is one of the principle outstanding problems in low- $x$ physics. The cross sections are large, of the order of $10 \%$ of the total DIS cross section. However, in contrast to standard DIS processes, the intact final state proton implies that no net colour charge is transferred between the scattering photon and the proton and hence that a minimum of two partons are exchanged in the $t$ channel. Describing such processes in QCD thus represents a significant field-theoretical challenge. Data obtained from HERA to date have led to considerable progress. The comparison of non-diffractive with diffractive DIS is a promising direction from which to approach the 


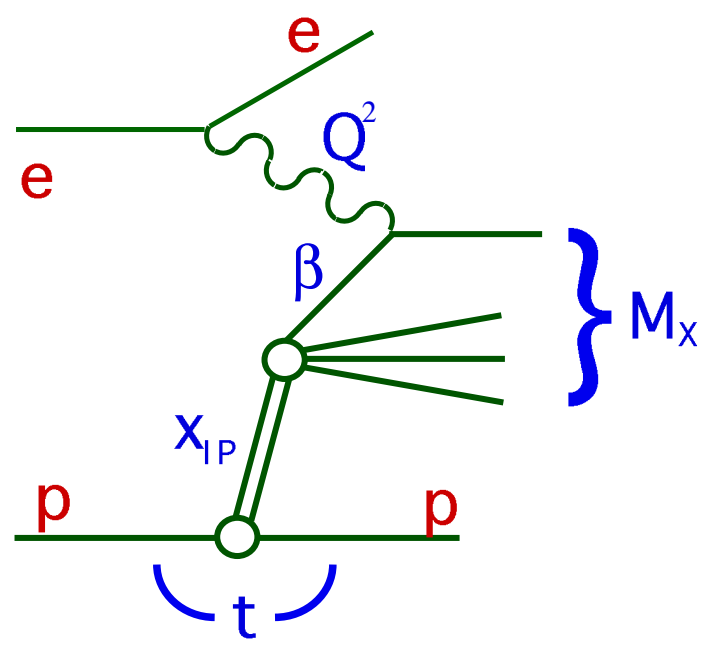

Figure 13: The generic diffractive process of the type $e p \rightarrow e X p$.

problem of confinement of quarks within hadrons, since they represent the cases where the proton is either violently blown apart (inclusive scattering) or remains intact having suffered only a small change in energy or momentum (diffractive scattering), in an otherwise rather similar process.

In this section, first studies of possible measurements of inclusive diffractive DIS cross sections with deuteron beams are presented. The related topics of diffractive vector meson production, Deeply Virtual Compton Scattering and diffractive dijet and charm production have yet to be investigated, though they are equally important to a full programme of research.

\subsubsection{Coherent diffraction from the deuteron}

Diffraction in $e A$ is believed to be a highly promising place to study high density QCD, with the likelihood of gluon saturation and the approach of the Gribov black body limit [52], where the diffractive cross section is equal to half of the total cross section. A review in the context of low $x$ physics can be found in [53]. Studies of coherent diffraction from the deuteron, $e d \rightarrow$ $e d X$, at HERA energies would represent a first essential step for a subsequent programme of research into diffractive $e A$ scattering, sketched in section 3.5, providing a critical reference point. Channels such as diffractive vector meson production would also be crucial for the study of colour transparency and opacity.

The VFPS spectrometer (section 5.2) will allow the detection and measurement of intact final state deuterons scattered with $x_{\mathbb{P}}$ and $t$ in an identical range to that measured for diffractively scattered protons in the $e p$ data from HERA II. Specifically, the acceptance is expected to be uniform and close to $100 \%$ in the range $0.011<x_{\mathbb{P}}<0.024$ and $|t|<0.25 \mathrm{GeV}^{2}$. For the same $x_{\mathbb{P}}$ range, the acceptance falls smoothly from $100 \%$ for $|t|>0.25 \mathrm{GeV}^{2}$ to about $20 \%$ at $|t|=0.8 \mathrm{GeV}^{2}$ [54]. By comparing the expected $350 \mathrm{pb}^{-1}$ of VFPS-tagged $e p \rightarrow e p X$ data from HERA II with the $e d \rightarrow e d X$ data from HERA III, it is thus possible to compare diffractive scattering from the proton and the deuteron for a fixed 4-vector of the diffractive exchange. Assuming factorisation of diffractive vertices, the dependence of the cross section on $Q^{2}$ and 
$\beta$ with $x_{\mathbb{P}}$ and $t$ fixed is expected to be identical. The coupling of the diffractive exchange to the proton is described by the variables $x_{\mathbb{P}}$ and $t$. The assumption of the exchange of a simple effective Regge pole (the phenomenological pomeron) has been remarkably successful in the description of diffractive HERA data taken to date. Under this assumption, the $x_{\mathbb{P}}$ dependence of proton and deuteron data would also be expected to be the same. On the other hand, the dependence on $t$ would be different in the two cases due to the different form factors of the proton and the deuteron. However, the changes in the $t$ dependence as other variables such as $x_{\mathbb{P}}$ are varied ("shrinkage") would be expected to be the same. The comparison between diffractive ed and $e p$ data would lead to highly sensitive tests of these factorisation properties, many of which have no sound basis in QCD. Figure 14a illustrates the possible measurements of the diffractive structure function $F_{2}^{D(4)}$, derived from the 4-fold differential cross section $d \sigma / d \beta d Q^{2} d x_{\mathbb{P}} d t$ for $350 \mathrm{pb}^{-1}$ of $e p$ running and $40 \mathrm{pb}^{-1}$ of $e d$ running under the crude assumption that the ratio of couplings of the diffractive exchange to the deuteron and to the proton is a fixed factor of 2 . The acceptance of the VFPS is included in the calculation, though only statistical errors are shown. Figure $14 \mathrm{~b}$ shows the expected sensitivity to a difference in the shapes of the $\beta$ dependences. At low $t$, the statistical sensitivity reaches the $1 \%$ level.

\subsubsection{Comparisons of diffraction from the neutron and the proton}

Comparing the elastic and diffractive scattering of real and virtual photons from protons and neutrons represents an informative environment for the testing of isospin symmetry and diffractive factorisation. Diffractive factorisation implies that the energy dependence of the diffractive exchange and its coupling to the photon are independent of the nucleon vertex. Isospin symmetry further requires that the couplings at the nucleon vertex are equal for protons and neutrons. Together, these laws thus imply that the quasi-elastic cross sections $\left(\right.$ e.g. $\left.\sigma_{\gamma^{(*)} p \rightarrow \rho^{0} p}, \sigma_{\gamma^{(*)} n \rightarrow \rho^{0} n}\right)$, diffractive dissociation cross sections $\left(\sigma_{\gamma^{(*)} p \rightarrow X p}, \sigma_{\gamma^{(*)} n \rightarrow X n}\right)$ and total ${ }^{6}$ cross sections $\left(\sigma_{\gamma^{(*)} p}^{\text {tot }}\right.$, $\sigma_{\gamma^{(*)} n}^{\text {tot }}$ ) should be equal. Although total cross section data exist for $p p$ and $p n$ scattering up to relatively large energies [55], the scattering of photons from the neutron has not been studied with centre-of-mass energies above $\sqrt{ } s_{\gamma n} \sim 20 \mathrm{GeV}$, see figure 1 and [56]. Testing the equivalences between diffractive cross sections for protons and neutrons with virtual photons, such that partonic structure is resolved, adds a new layer of interest. Differences between the diffractive DIS cross sections in $e p$ and $e n$ scattering would imply a local flavour asymmetry in the diffractive parton densities, with similar implications to the $\bar{d}-\bar{u}$ asymmetries observed in inclusive DIS [2].

Running with deuterons at $E_{d}=920 \mathrm{GeV}$ is equivalent to running with protons and neutrons of $E_{p, n}=460 \pm 30 \mathrm{GeV}$, where the uncertainty arises from the Fermi motion of the nucleons within the bound state (see figure 15). It therefore represents similar (if somewhat less controlled) conditions to an $e p$ run with the proton beam energy reduced to half of its nominal value. Comparing diffractive data from an ep run with $E_{p}=460 \mathrm{GeV}$ and an $e d$ run with $E_{d}=920 \mathrm{GeV}$ would allow an unprecedented study of the effects of nuclear binding on diffractive cross sections.

\footnotetext{
${ }^{6}$ For the total cross section, this is not true when the virtual photon interacts electromagnetically with the valence quarks in the nucleon.
} 

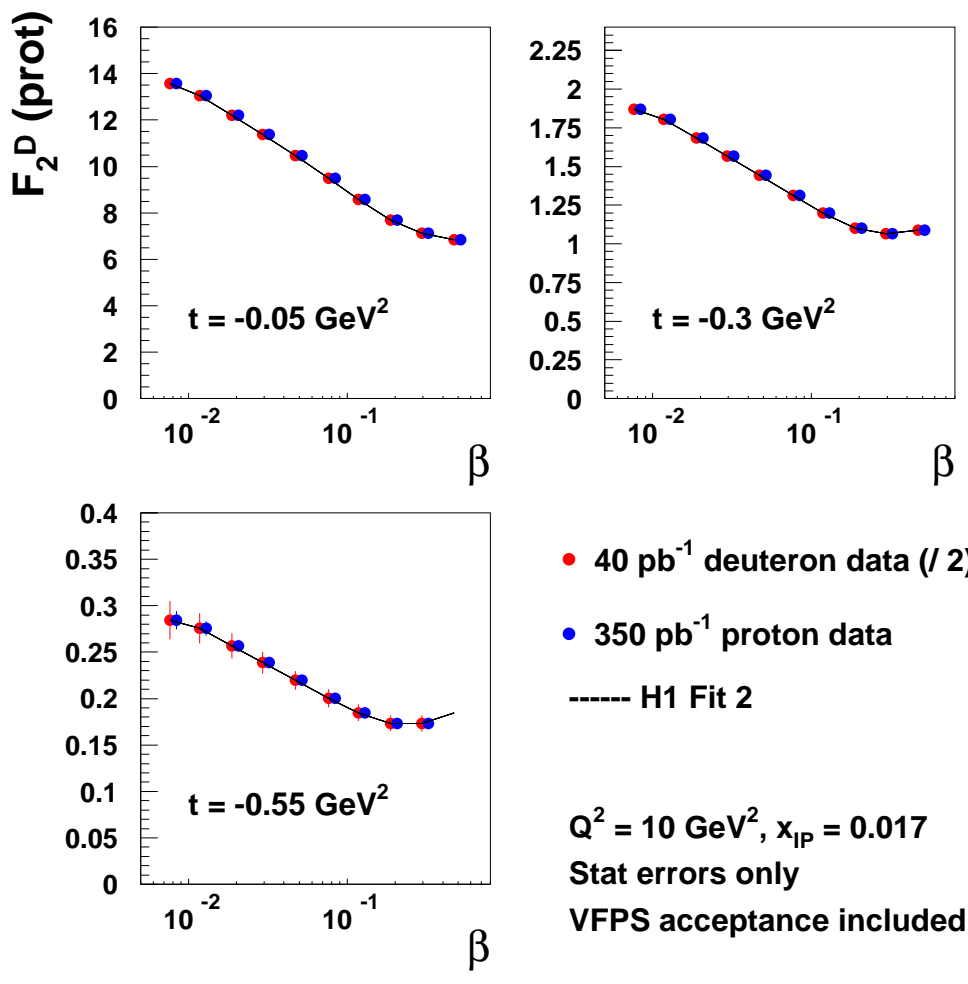

- $40 \mathrm{pb}^{-1}$ deuteron data (/ 2)

- $350 \mathrm{pb}^{-1}$ proton data

----- H1 Fit 2

$Q^{2}=10 \mathrm{GeV}^{2}, \mathrm{x}_{\mathrm{IP}}=0.017$

Stat errors only

VFPS acceptance included

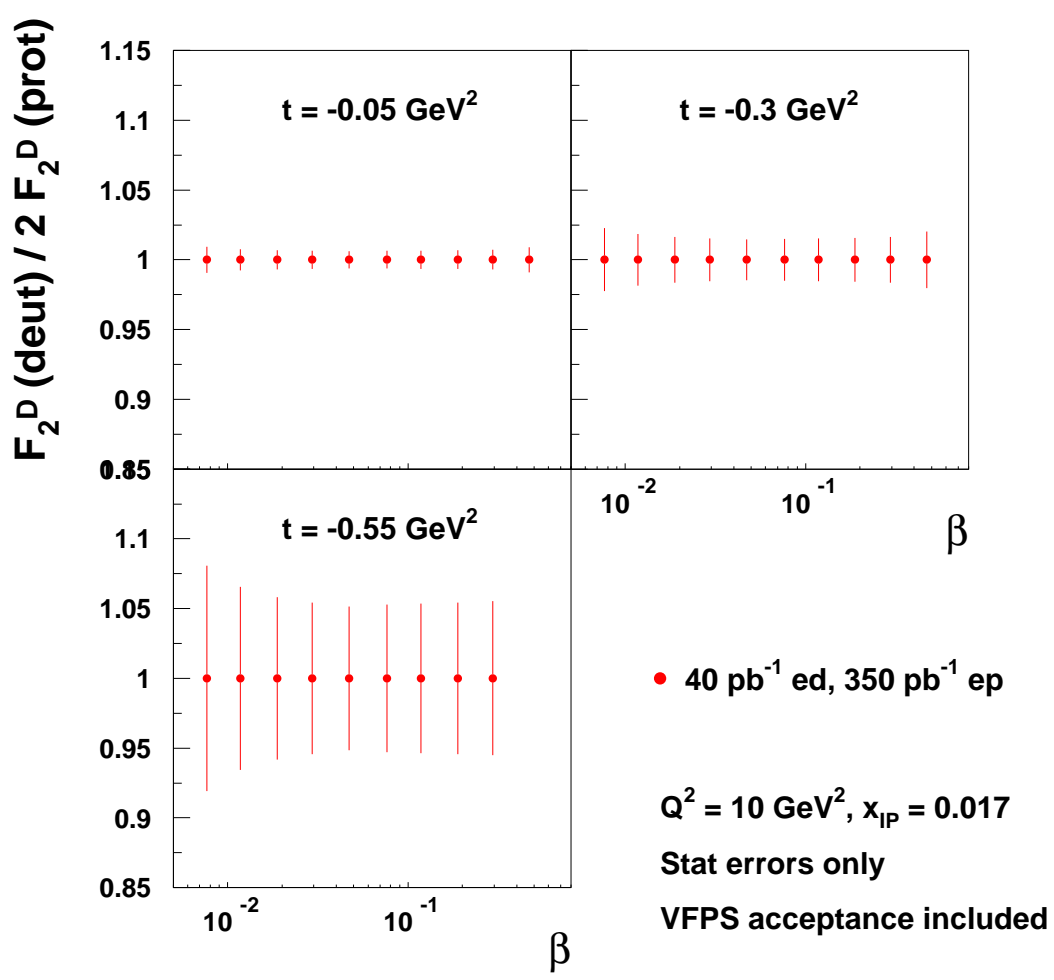

Figure 14: Simulated measurements of the diffractive structure function $F_{2}^{D(4)}$ from diffractive $e d$ and $e p$ scattering using the VFPS. The deuteron structure function is crudely assumed to be a constant factor of 2 larger than that of the proton. Upper plots, $F_{2}^{D}$ for the proton and for the deuteron, the latter scaled by a factor of 2 . Lower plots, ratio of the deuteron and proton results. The data are integrated over the acceptance range of the VFPS in $x_{\mathbb{P}}$ and $t$ and are integrated over $Q^{2}$ for visibility. 

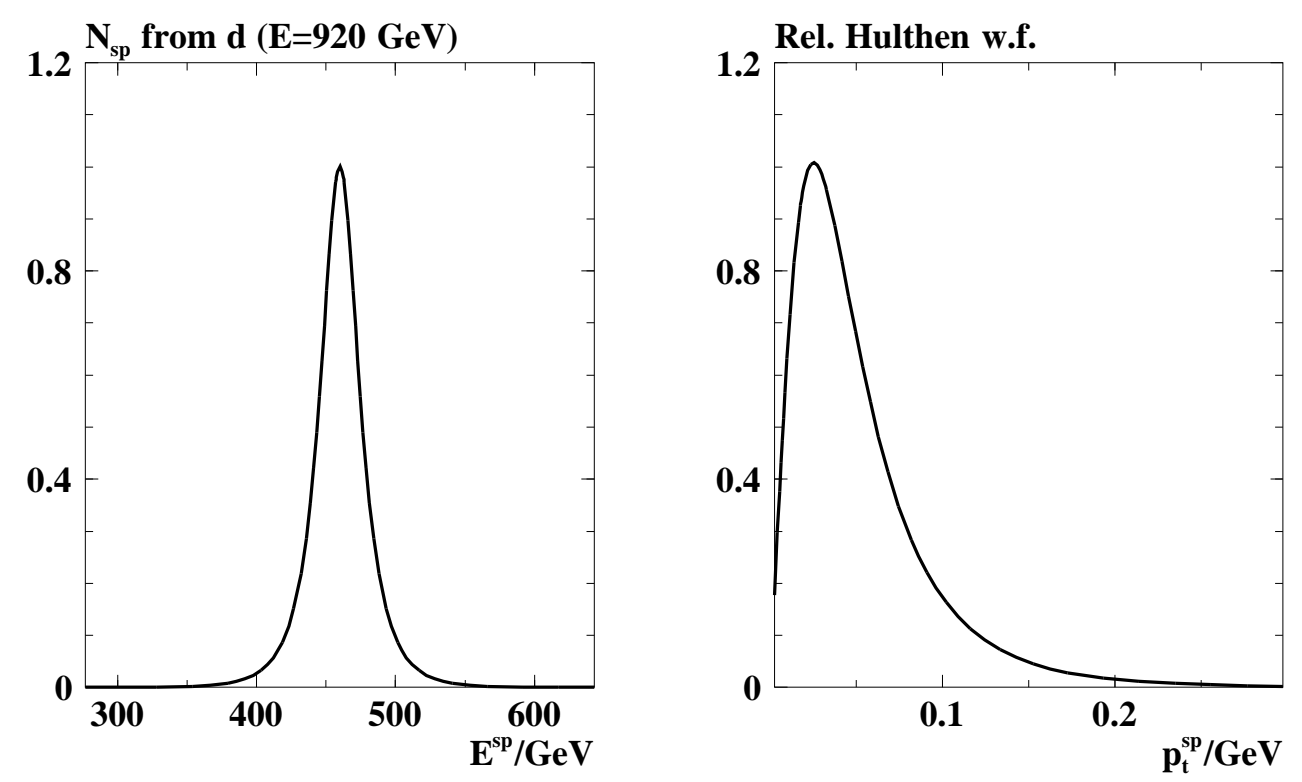

Figure 15: The energy and transverse momentum distributions of the spectator nucleons from the deuteron in the laboratory system, calculated using the known deuteron wave function [60]. To a good approximation, $|t|=p_{t}^{s p^{2}}$.

As an example, figure 16 shows the sensitivity to a difference between $F_{2}^{D}$ as measured from the proton and the neutron integrated over $t$ and $Q^{2}$ for different values of $\beta$ and $x_{\mathbb{P}}$ assuming it is possible to cleanly separate diffractive scattering from the neutron and the proton on an event by event basis. With $40 \mathrm{pb}^{-1}$ of $e d$ data, differences at the level of $1 \%$ may be detected if systematic errors can be tightly controlled.

As discussed in section 5.2, even with perfect proton and neutron tagging detectors, it will not be possible to determine event by event whether a final state nucleon with around half the beam energy originates from diffractive scattering off the proton or the neutron. The very different $t$ distributions of diffractively scattered nucleons and spectator nucleons should allow a relatively clean separation, but a detailed study has yet to be performed. Measuring vector meson cross sections leads to a cleaner separation, since the simple final states arising from decays such as $\rho^{0} \rightarrow \pi^{+} \pi^{-}$and $J / \psi \rightarrow \mu^{+} \mu^{-}$can be measured accurately in the central tracking detectors, leading to a precise independent measurement of $t$. Comparing this value of $t$ from the vector meson decay with the values measured from leading proton and neutron taggers should allow a much improved separation efficiency.

Reducing the nucleon beam energy modifies the acceptance regions of the detectors as a function of the kinematic variables, opening up new kinematic regions for study. Examples in the diffractive area are extensions to lower $W$ for vector meson production cross sections and to larger $x_{\mathbb{P}}$ or $\beta$ for $F_{2}^{D(4)}$ measurements [57]. Reduced $E_{p}$ running would also make possible a direct extraction of $F_{L}^{D(4)}$, through the comparison of $F_{2}^{D(4)}$ data at fixed $x, Q^{2}$ and $x_{\mathbb{P}}$, but different $y$ values. Studies [58] have shown that a $40 \%$ differential measurement of $F_{L}^{D(4)}$ with comparable statistical and systematic errors would be possible with $50 \mathrm{pb}^{-1}$ at $E_{p}=500 \mathrm{GeV}$ and $250 \mathrm{pb}^{-1}$ at $E_{p}=820 \mathrm{GeV}$. 


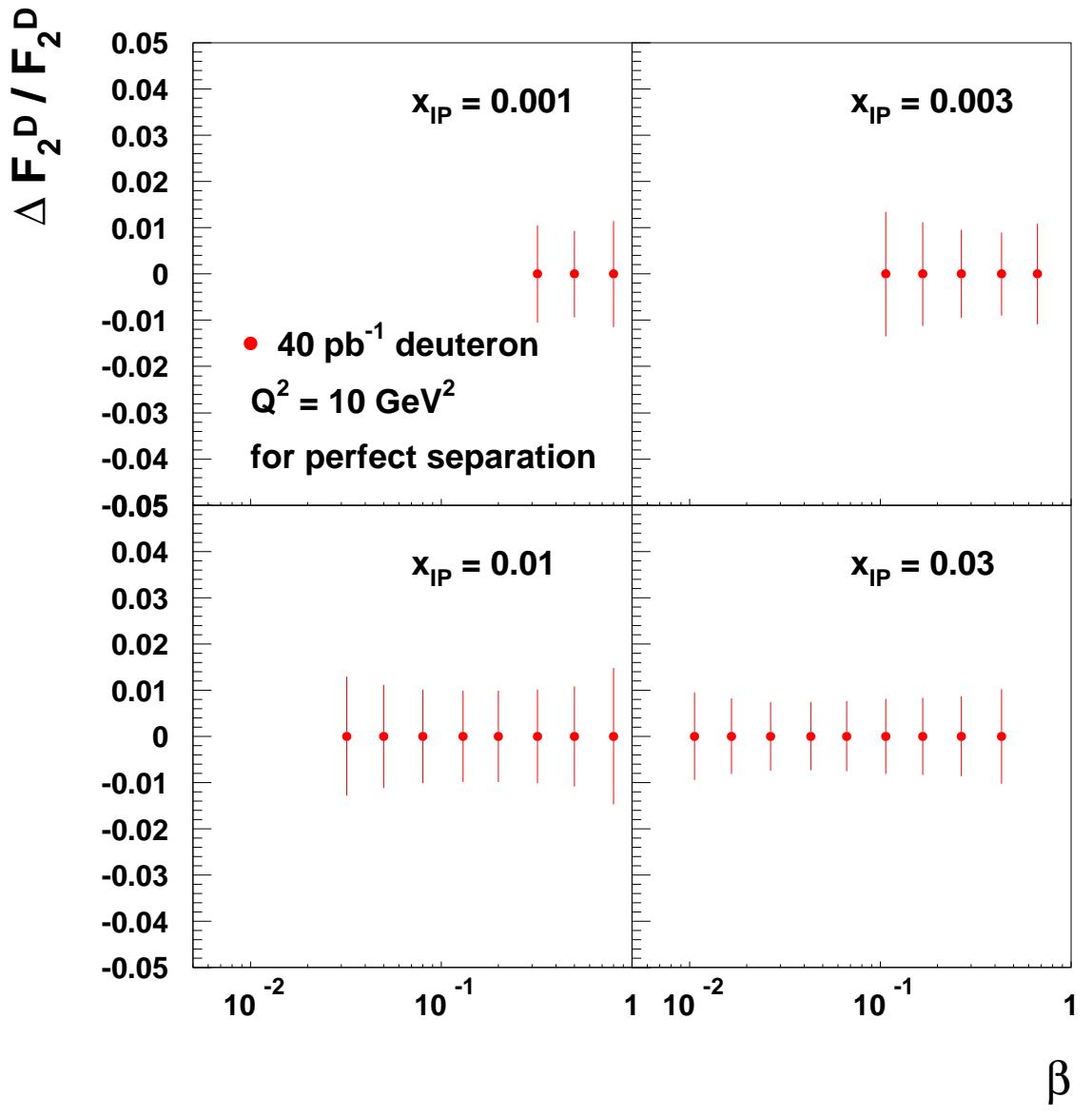

Figure 16: Simulated measurement of the scaled difference in $F_{2}^{D}$ between $e p$ and $e n$ scattering, assuming perfect separation of the two processes. The data are integrated over $|t|<1 \mathrm{GeV}^{2}$ and the approximate $Q^{2}$ coverage of the $\mathrm{H} 1$ detector. 


\subsubsection{Vector meson production}

Experiments with deuteron beams can be used to explore the effects of colour transparency and colour opacity via the study of coherent processes with the deuteron, for example production of vector mesons in the reaction $\gamma^{*}+d \rightarrow V+d$ at large enough $t$. The study of the $t$ dependence of this process at $-t \geq 0.5 \mathrm{GeV}^{2}$ can provide a sensitive test of leading and higher twist shadowing effects [59]. 


\section{Possible Further Studies}

\section{Introduction}

The following sections are concerned with the questions that may be addressed using HERA and $\mathrm{H} 1$ beyond the electron-deuteron programme that is the immediate subject of this Letter of Intent. These questions include further investigations of some of the phenomena already observed at HERA, such as the interesting behaviour observed in the inclusive $e p$ cross section at scales corresponding to about one third of the proton's size, studies of the final state in this region, further investigation of some of the phenomena described above, such as shadowing in nuclei heavier than deuterium and also studies of areas of QCD that are currently completely unexplored, for example the distribution of parton spin at small values of Bjorken $x$.

\section{Low $x$ Physics and QCD at High Parton Density}

\subsection{Introduction}

Although the lagrangian of QCD is known, the non-linearity of the resulting equations implies that the ground state, the vacuum, is highly non-trivial, with properties analogous to those of a super-fluid or a super-conductor. Thus, as in such media, the relevant degrees of freedom are not the fundamental quarks and gluons which figure in the lagrangian but the hadrons within which these are confined. These "quasi-particles" must result from the collective behaviour of quarks and gluons, behaviour which is currently not understood. It is generally believed that it is the complex QCD vacuum, containing both gluon and quark, or chiral, condensates, which leads to confinement, a successful description of which would represent a major step forward in our understanding of the forces of nature.

A particularly promising place to obtain information on the transition from the "hard" world of quarks, gluons and perturbative QCD to the "soft" world of the hadrons is electron-proton scattering at the highest energies, since the scale of the interaction can be adjusted by varying the virtuality of the interacting photon. Indeed, it is possible that a first experimental hint of a scale associated with the new phenomena in the vacuum has already been observed at HERA.

As mentioned in section 2.1, the HERA experiments discovered that the structure function $F_{2}^{p}\left(x, Q^{2}\right)$ rises sharply with decreasing $x$ in the deep inelastic region, indicating directly that the density of quarks in the proton increases as the proportion of the proton's momentum they carry decreases and less directly that the gluon density behaves similarly. The steepness of the rise of $F_{2}^{p}\left(x, Q^{2}\right)$ is seen to be dependent on $Q^{2}$, the scale at which the proton is probed; higher values of $Q^{2}$, i.e. smaller distance scales, are associated with a steeper rise of $F_{2}^{p}\left(x, Q^{2}\right)$. At low $x$, the behaviour of this structure function can be parameterised using the functional form $F_{2}^{p}\left(x, Q^{2}\right)=c\left(Q^{2}\right) x^{-\lambda\left(Q^{2}\right)}$ [61]. As expected from the discussion above, $\lambda$ increases with increasing $Q^{2}$. Figure 17, shows that this variation is linear in $\ln \left(Q^{2}\right)$. Interestingly, the behaviour of $\lambda$ with $Q^{2}$ changes at a scale of about $0.5 \mathrm{GeV}^{2}$, corresponding to distances of about $0.3 \mathrm{fm}$ or one third of the proton's radius. At roughly the same scale, the gluon density extracted from 


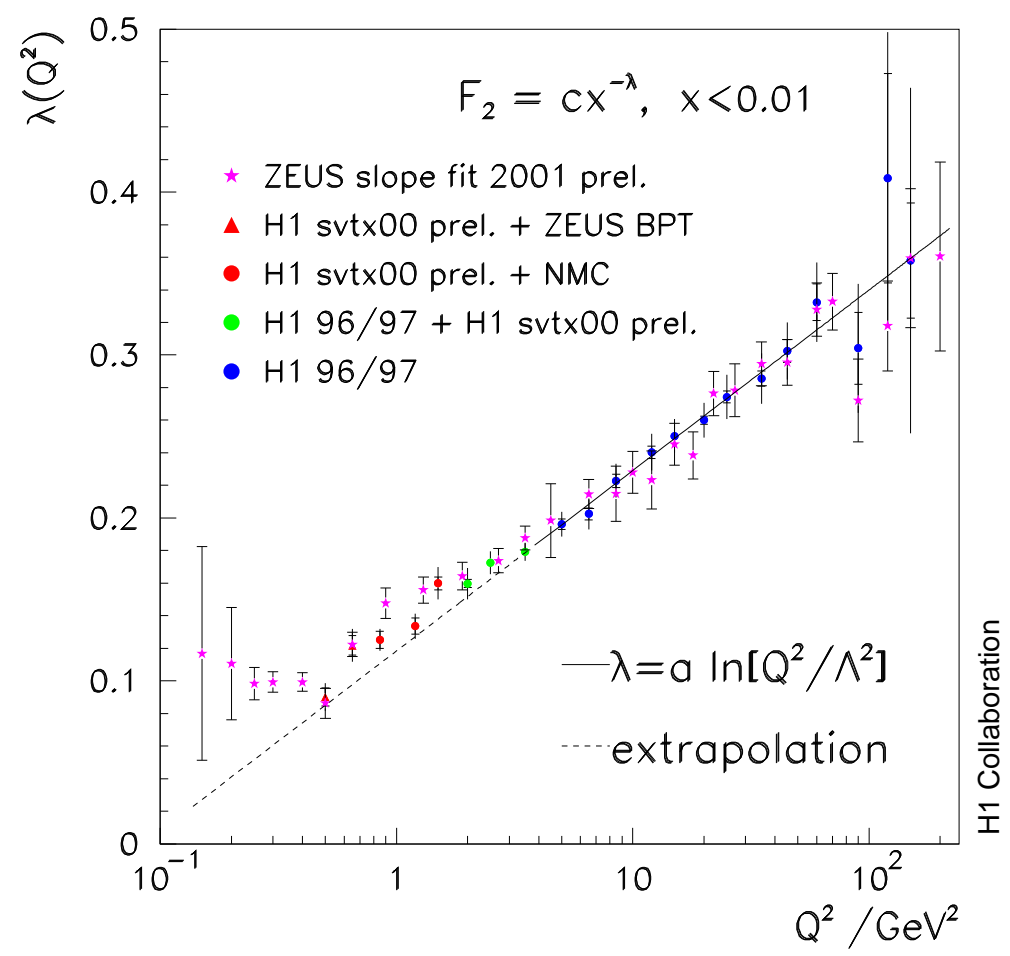

Figure 17: The quantity $\lambda\left(x, Q^{2}\right)$ extracted from parameterisations of the H1 and ZEUS structure function measurements using the form $F_{2}^{p}\left(x, Q^{2}\right)=c\left(Q^{2}\right) x^{-\lambda\left(Q^{2}\right)}$.

DGLAP [62-65] based fits to the $F_{2}^{p}\left(x, Q^{2}\right)$ data also behaves peculiarly, becoming small or even negative for low $x$ values in some analyses. As $F_{L}^{p}\left(x, Q^{2}\right)$ is closely related to the gluon density, its measurement will allow a more direct study of the behaviour of the gluons in the proton at scales around $Q^{2}=1 \mathrm{GeV}^{2}$.

The data obtained in measurements of proton structure at this scale may help us to better understand the QCD vacuum or perhaps to forge a link between the partons of perturbative QCD, with $u, d$ and $s$ quarks of mass about 3,6 and $120 \mathrm{MeV}$, respectively [1], and the "constituent" quarks which can explain, among other things, the pattern of baryon masses if they are assumed to behave as spin $\frac{1}{2}$ particles with $u_{c}$ and $d_{c}$ masses of $363 \mathrm{MeV}$ and an $s_{c}$ mass of $538 \mathrm{MeV}$ [66].

In the following we discuss first the inclusive measurements that may be made in this region and then move on to consider what may be learned from studies of the hadronic final state.

\subsection{Gluons at low $x$ and $Q^{2}$ and the measurement of $F_{L}^{p}$}

The HERA measurements of the inclusive $e p$ scattering cross section at low $x$ have revealed a steep rise of the proton structure function $F_{2}$ with $Q^{2}$. The approximate relation [67]

$$
\frac{\partial F_{2}\left(x / 2, Q^{2}\right)}{\partial \ln Q^{2}} \simeq \frac{10}{27} \frac{\alpha_{s}\left(Q^{2}\right)}{\pi} \cdot x g\left(x, Q^{2}\right)
$$

therefore suggests that the gluon distribution at low $x$ is large, see figure 18. A very high gluon 


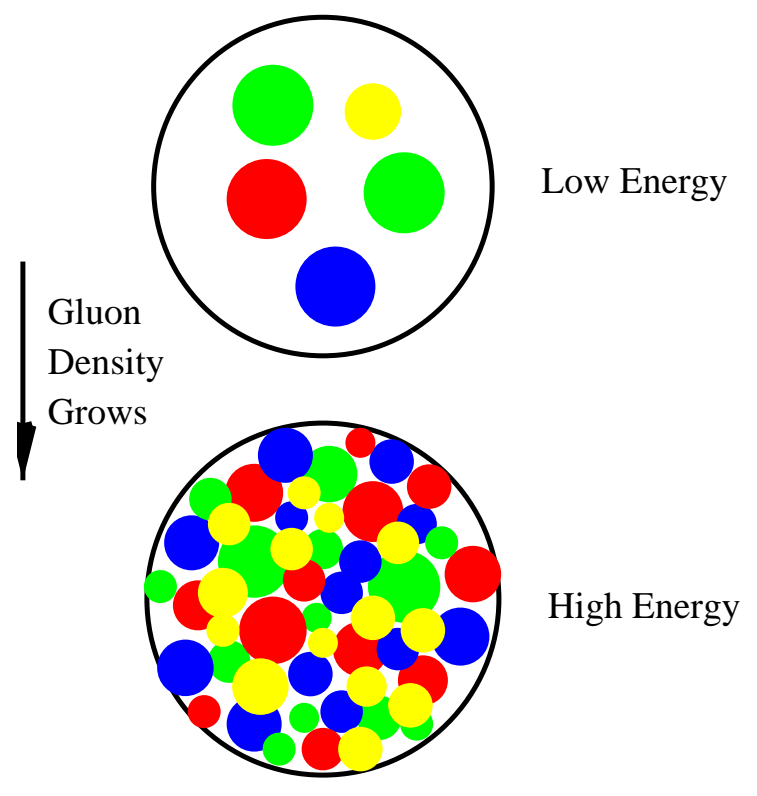

Figure 18: Rise of the gluon density in the nucleon for decreasing $x$, from [68].

density, in the region of small coupling, is characteristic for a new phase of matter which is often termed the "colour glass condensate" [68] because coloured gluons form a disordered condensate state. At large densities, at a $Q^{2}$ scale of about

$$
Q_{s}^{2} \simeq \alpha_{s} N_{c} x g \frac{1}{\pi R^{2}}
$$

the parton distribution functions at fixed $Q^{2}$ are expected to saturate. For pQCD this raises the problem as to where the standard evolution equations are expected to fail, see for example $[69,70]$. The behaviour of $x g$ in this region is interesting in its own right, but it is also of phenomenological importance as $x g$ is necessary for predicting a large variety of high energy cross sections, including, for example, the possibility of Higgs boson production in pomeronpomeron scattering at the $\mathrm{LHC}$, i.e. in a rather clean event configuration caused by multi-gluon exchange. Further, the high density states in $A A$ interactions cannot be interpreted without having measured the parton distributions in $e p$ and $e A$ scattering.

In this regard the transition region from DIS to photoproduction, $Q^{2} \sim 1 \mathrm{GeV}^{2}$, is of particular interest because the saturation scale is estimated to be of that order, see for example [68]. Moreover, the puzzling observation has been made in many DGLAP pQCD analyses that in this region the simple approximation of equation 14 fails: the gluons tend to disappear at low $x$ and the persistent rise of $F_{2}^{p}$ to low $x$ has to be caused by sea quarks, which will be studied in ed scattering at HERA. This dramatic change in the gluon and sea quark distributions for $Q^{2}$ near $1 \mathrm{GeV}^{2}$ is illustrated in figure 19 . Although similar behaviour was observed in many other analyses, $x g$ in this region is far from being understood, different analyses yielding very different predictions, some even preferring $x g$ to be negative at low $x$ and $Q^{2}$ [71].

The longitudinal structure function $F_{L}^{p}\left(x, Q^{2}\right)$, together with $F_{2}^{p}$, provides a discriminative test of perturbative QCD which is thought to be decisive at low $x$. In the QPM and for spin 

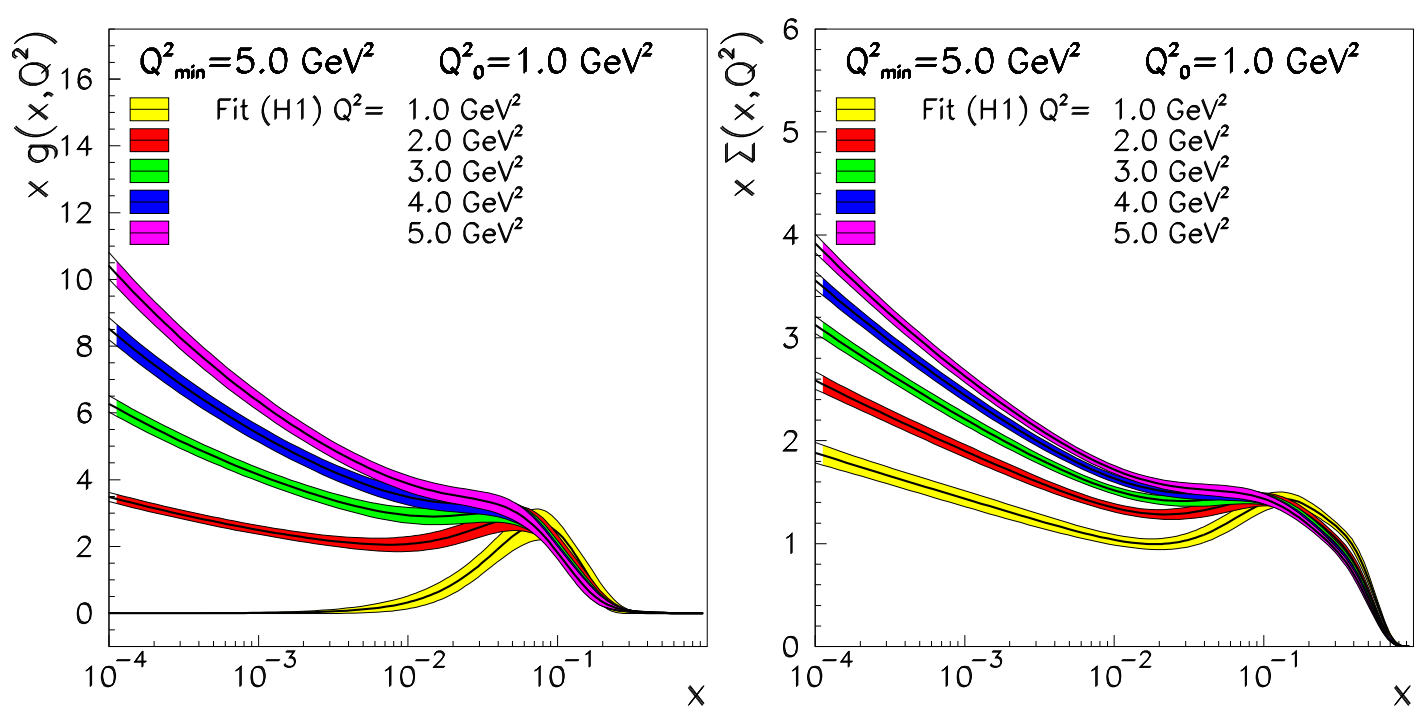

Figure 19: Variation of the gluon distribution (left) and the quark singlet distribution (right) in a QCD NLO analysis of the H1 inclusive cross section data. As can be seen, in this study, the gluon vanishes at low $x$ for $Q^{2}=1 \mathrm{GeV}^{2}$ while it dominates the low $x$ behaviour of $F_{2}$ at $Q^{2}$ values larger than a few $\mathrm{GeV}^{2}$.

$\frac{1}{2}$ quarks with negligible transverse momenta $F_{L}^{p}=0$ [72]. In QCD, however, $F_{L}^{p}$ acquires a non-zero value because of gluon radiation which allows access to $x g$ via the relation [73]

$$
F_{L}=\frac{\alpha_{s}}{4 \pi} x^{2} \int \frac{d z}{z^{3}} \cdot\left[\frac{16}{3} F_{2}+8 \sum Q_{q}^{2}\left(1-\frac{x}{z}\right) g\right]
$$

written here for four flavours. Thus another constraint on $x g$ is obtained.

Moreover, as is seen in figure 20, very large effects are expected from higher order corrections which make predictions for $F_{L}^{p}$ and $x g$ uncertain. This also influences the possibility of observing higher twist effects in $F_{L}^{p}$ which are suppressed in $F_{2}^{p}$ [74]. Without a precision measurement of $F_{L}^{p}$, a respectable theory at low $x$ cannot be established.

A measurement of $F_{L}^{p}\left(x, Q^{2}\right)$ can be obtained from a high precision cross section measurement extending to the largest possible inelasticity values $y \rightarrow 1$ for which the reduced DIS cross section

$$
\sigma_{r}=F_{2}\left(x, Q^{2}\right)-\frac{y^{2}}{1+(1-y)^{2}} F_{L}\left(x, Q^{2}\right)
$$

tends to $F_{2}^{p}-F_{L}^{p}$. A high $y$ cross section measurement thus leads to a potentially very accurate determination of the longitudinal structure function.

So far, access to the longitudinal structure function has been limited since the inclusive DIS cross section contains both $F_{2}^{p}$ and $F_{L}^{p}$ which can only be separated by varying the beam energy, preferably $E_{p}$, as discussed in [75], or by making assumptions about the behaviour of $F_{2}^{p}$ at large $y$. Because at HERA a much larger $y$ range is available than in fixed target experiments, this latter technique makes possible an extraction of $F_{L}^{p}$, as has been done by the H1 Collaboration [76]. These determinations of $F_{L}^{p}$, however, are confined to the highest $y$ and have a modest 

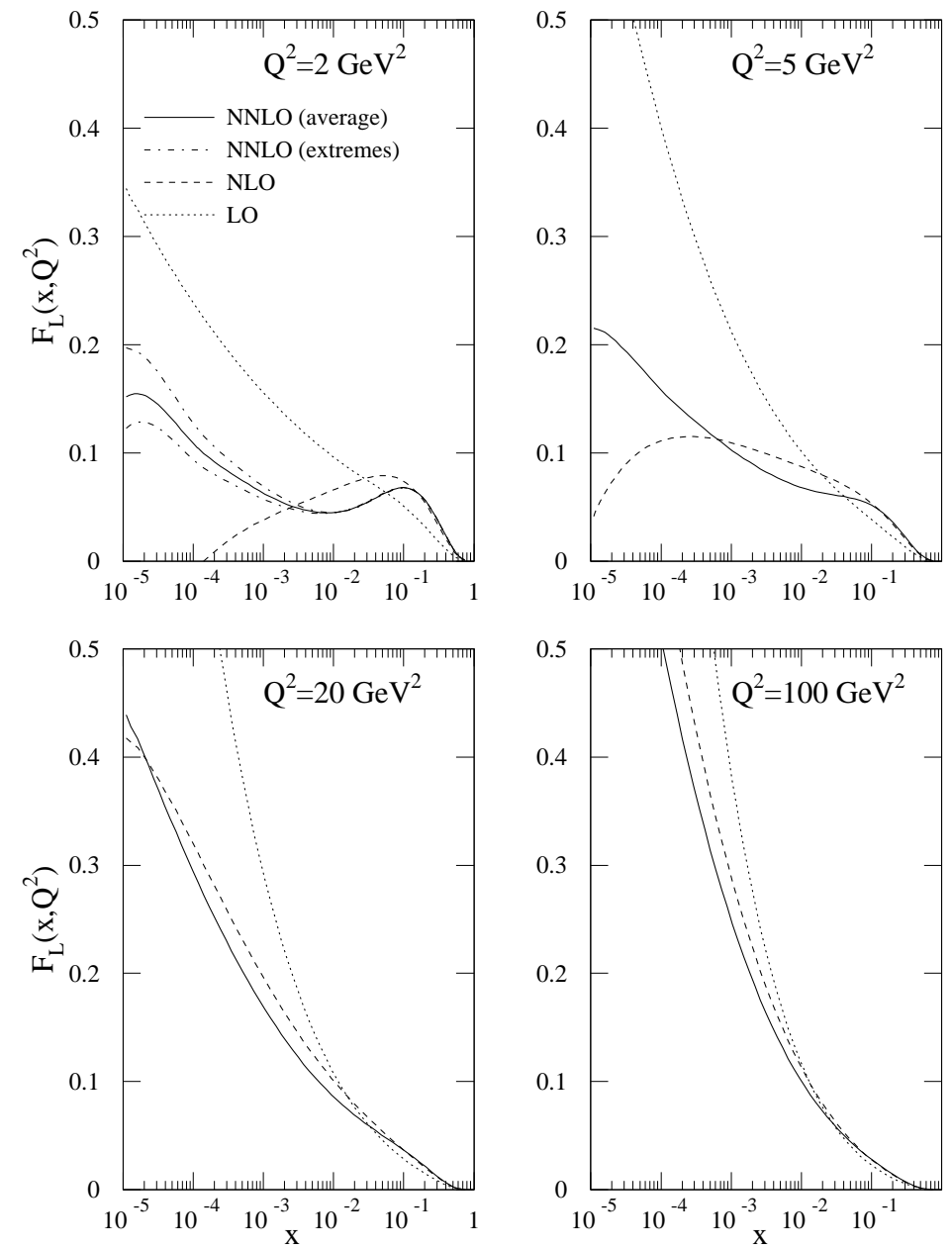

Figure 20: Effects and uncertainties of higher order $\mathrm{pQCD}$ on the behaviour of the longitudinal structure function, from [71]. 
accuracy of the order of $20 \%$. The $x$ dependence of $F_{L}^{p}$ cannot be measured at HERA without varying the beam energy, and only this method is free of assumptions on the behaviour of $F_{2}^{p}$. A challenging level of experimental precision is required. For the presently inaccessible region in which the transition from $\mathrm{pQCD}$ to photoproduction occurs, where the uncertainty of the gluon distribution is most striking, a full simulation was performed of a possible measurement of $F_{L}\left(x, Q^{2}\right)$ using the new backward spectrometer, is described in section 5.5.

For a set of 3 beam energies, 920,500 and $400 \mathrm{GeV}$, and luminosities of $5 \mathrm{pb}^{-1}$, the resulting accuracy of the $F_{L}^{p}$ measurements is shown in figure 21 and compared with the new preliminary result from the data obtained in 2000 in a shifted vertex run. As can be seen, $F_{L}^{p}$ becomes measurable rather precisely and independently of any assumption on the other structure function ${ }^{7}$.

\subsection{Measurements in the forward region}

The experience from the first phase of HERA running, HERAI, is that some effects are more clearly revealed in studies of the hadronic final state than in inclusive measurements; deviations from "standard" QCD model predictions can then be enhanced.

In the following, three poorly understood areas are discussed, parton branching, the QCD vacuum and non-perturbative particle production.

\subsubsection{Parton branching}

The basic evolution equations for parton branching, the DGLAP and BFKL equations [7779], are expected to be valid in certain regions of phase space only. The CCFM [80-83] and LDC [84-87] formalisms combine in a consistent way some features of the DGLAP and BFKL evolution equations and should be superior to both of them when details of the final state are investigated. However, as all of them are approximations to the complete and complicated parton branching process, they are all expected to show deficiencies when sufficiently precise tests are performed.

It has been observed that improving the CCFM/LDC splitting function to include the DGLAPlike (non-singular) terms results in a worse description of the initial state branching processes. This failure indicates that our understanding and modelling of branching processes is far from satisfactory. This may indicate that multiple parton exchange mechanisms are at work and have to be included. In the BFKL and CCFM/LDC description bare gluons are replaced by so-called reggeised gluons, i.e. gluons which are "dressed" to include the effects of virtual corrections. This approach is, however, just the first step towards the full consideration of multi-parton exchange processes, including the exchange of multiple ladder cascades. In this scenario, a more advanced theoretical method than the conventional parton level perturbative calculation is needed.

\footnotetext{
${ }^{7}$ For larger $Q^{2}, F_{L}^{p}$ can be measured with the current $\mathrm{H} 1$ detector when the beam energy is varied. This measurement uses the high precision backward silicon tracker and the SpaCal calorimeter in conjunction with the reconstruction of the energy momentum balance using the H1 LAr calorimeter and tracking.
} 

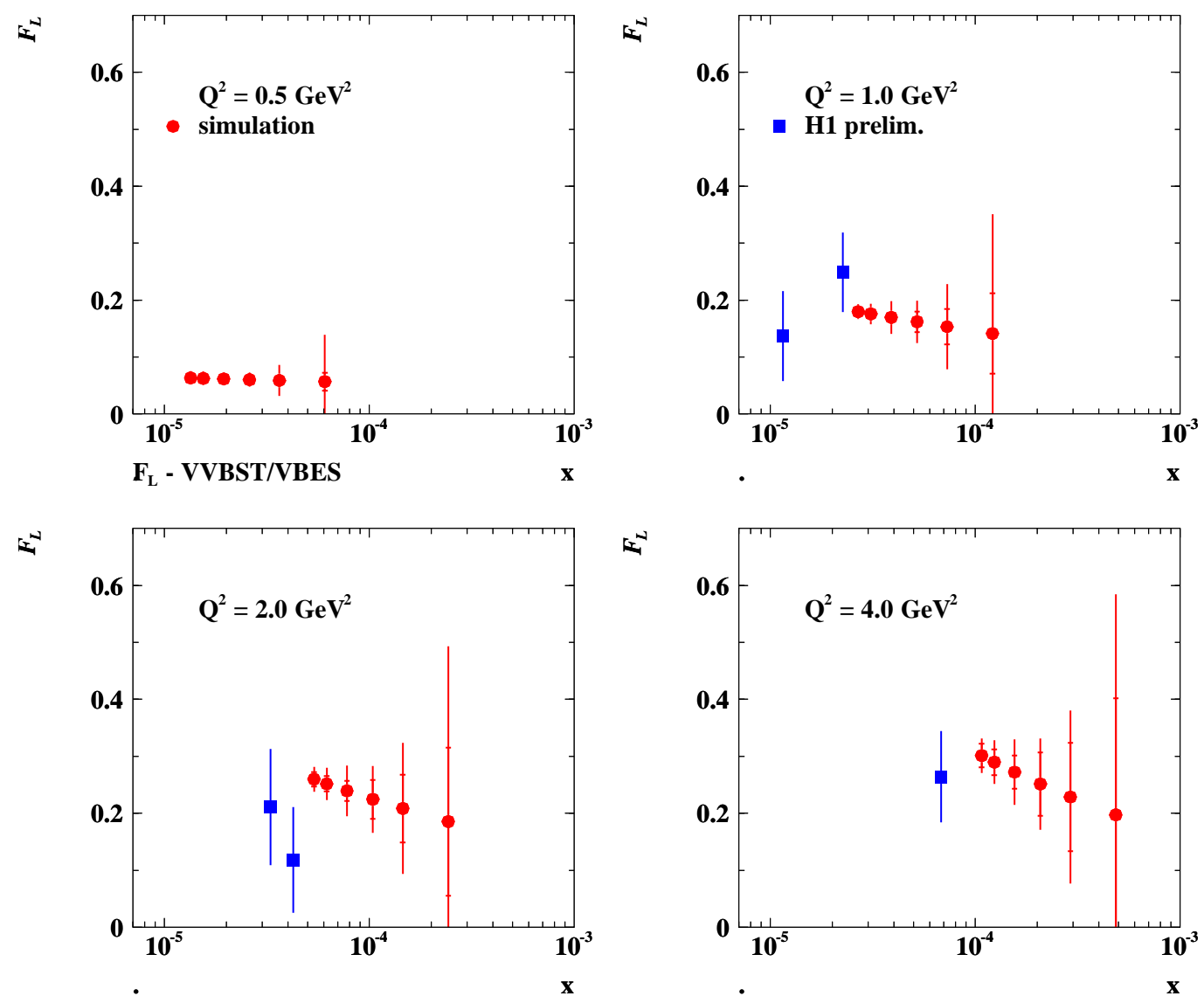

Figure 21: Simulation of the measurement of the longitudinal structure function $F_{L}^{p}\left(x, Q^{2}\right)$ using the upgraded backward apparatus operated during a series of runs with different proton beam energy. The inner error bar is the statistical error and the full error bar the quadratic sum of the statistical and systematic errors, taking into account the correlation of the systematic errors of the different measurements. This measurement can be performed at higher $Q^{2}$ values using the existing $\mathrm{H} 1$ apparatus and is thus not simulated here for this kinematic region. The square points represent the preliminary and best low $Q^{2}$ data of $\mathrm{H} 1$ obtained in an analysis in which the behaviour of the two structure functions, $F_{2}$ and $F_{L}$, is parameterised and determined simultaneously. 


\subsubsection{The QCD vacuum}

The vacuum state in QCD has a highly non-trivial structure, and contains both a gluon condensate and a quark, or chiral, condensate. Although the magnitude of both gluon and quark condensates present in the QCD vacuum can be estimated to a fair degree, the more detailed properties of the vacuum state are still unknown. As mentioned above, these properties are thought to be responsible for the phenomenon of confinement. They also control the nonperturbative hadronisation process and affect the properties of reactions with vacuum quantum number exchange, i.e. the nature of the pomeron and soft and hard diffraction, one of subjects of particular interest at HERA.

In the string hadronisation model the gluon condensate is treated as a dual super-conductor [88], and particle production is described as a tunnelling process in a flux tube or a vortex line. In the "Disoriented Chiral Condensate" the mechanism responsible for particle production is instead related to the quark condensate $[89,90]$. It is possible that both mechanisms contribute in nature, perhaps in different ratios in different reactions. To resolve these questions more detailed data are needed for charge and $p_{t}$ correlations, $K / \pi$ ratios, etc. We also note that some experiments indicate differences between hadronisation in $e^{+} e^{-}$annihilation and in DIS or hadronic collisions, as is discussed further below.

If the nucleon is associated with a topological singularity in the pion field, as in the Skyrme model, the vacuum properties should influence not only diffractive scattering, but also peripheral collisions with pion exchange. These effects can be detected at HERA III.

\subsubsection{Non-perturbative particle production}

Collisions between electrons and deuterons allow a comparison of the properties of protons and neutrons. The fragmentation region of the proton- or neutron-remnant has never been studied in detail. The primordial $k_{t}$ distribution of partons in the nucleon is poorly known, although some measurements indicate the need for relatively large intrinsic $k_{t}$. However, these measurements cannot distinguish whether the large $k_{t}$ needed is intrinsic to the colliding hadron or a result of the parton branching process.

The universality of jet fragmentation is a basic assumption made in most theoretical approaches to the hadronisation process. However, some experimental analyses indicate that the ratio of strange to non-strange quark production observed in $e^{+} e^{-}$annihilation is not the same as that seen in DIS or hadronic collisions. It is therefore very interesting to investigate whether there are differences in the hadronisation when going from the proton fragmentation region in DIS, where behaviour similar to that observed in hadron-hadron scattering is expected, to the central region, which is expected to be very similar to $e^{+} e^{-}$annihilation. These studies could involve the measurement of kaon, baryon, and pion production as a function of rapidity.

It should be pointed out that, although the target region was studied in some detail by the EMC experiment, the $x$ values attainable were not small enough to properly investigate the perturbative QCD branching process; the available centre-of-mass energy was too small.

Since QCD processes will create a significant background in the search for signals of new physics it is obvious that a better understanding of the parton branching processes, the QCD vacuum and the universality of fragmentation is necessary. 


\section{ep scattering}

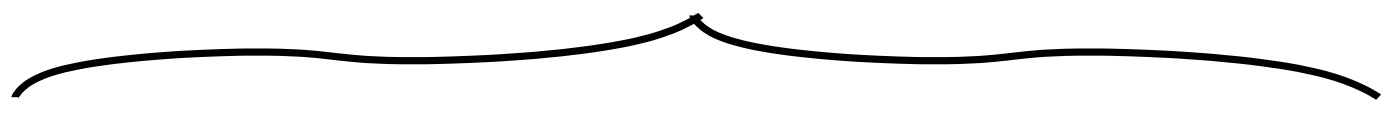

$\mathrm{e}^{+} \mathrm{e}^{-}$region

p $\overline{\mathbf{p}}$ region

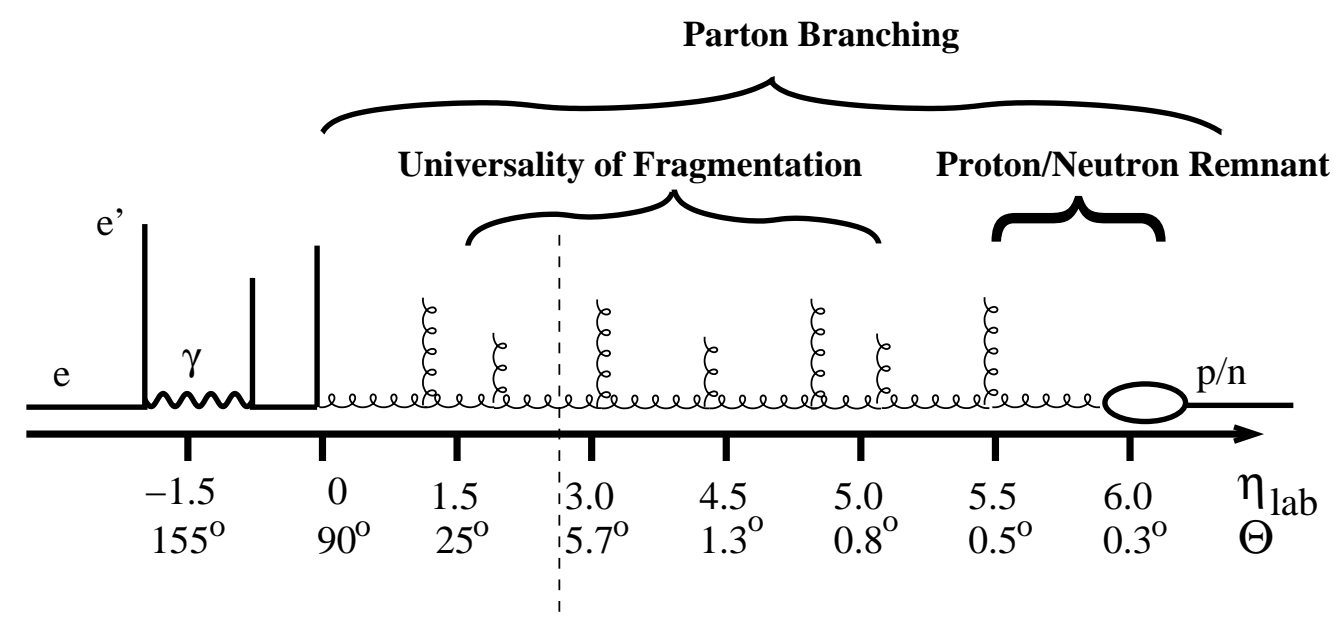

acceptance limit: HERA 1,2

Figure 22: The rapidity range accessible in the HERA laboratory frame together with the regions in which the fragmentation process is expected to behave in a manner similar to $e^{+} e^{-}$ annihilation and hadron-hadron collisions. 


\subsubsection{The experimental situation}

The hadronic final state in $e^{+} e^{-} \rightarrow q \bar{q}$ is precisely measured from low energy experiments up to the highest possible LEP energies. The underlying QCD processes are calculated in perturbation theory up to $\mathcal{O}\left(\alpha_{s}^{2}\right)$. The partonic final state is supplemented by higher order QCD radiation in form of leading log parton showers and is then fragmented into visible hadrons via nonperturbative hadronisation models, like PYTHIA [91] or HERWIG [92]. These hadronisation models are tuned to describe the final state in $e^{+} e^{-}$reactions.

The situation in $p \bar{p}$ reactions is completely different. Whereas the underlying hard scattering processes can be calculated in perturbation theory, the description of the hadronic final state is more complicated, as the incoming partons, quarks and gluons, undergo initial state branching processes, which are initiated by partons in the non-perturbative phase of the collision.

The hadronic final state in $e p$ reactions forms a bridge between the well understood situation in $e^{+} e^{-}$annihilation and the less-well understood situation in $p \bar{p}$ collisions. In the rapidity region of the virtual (quasi-real) photon, the hard scattering process is expected to be similar to the $e^{+} e^{-}$case, whereas moving towards the fragmentation region of the proton, the situation is similar to that in $p \bar{p}$ collisions. One of the advantages of $e p$ scattering is the possibility it offers to cover the full range from the photon fragmentation region, which can be described by perturbative QCD, to the proton fragmentation region, which might be described by collective phenomena only.

Multiple scattering processes are essential for a reasonable description of QCD processes in $p \bar{p}$ reactions. However, due to the complicated nature of these processes, it would appear to be nigh on impossible to study these effects in detail in $p \bar{p}$ collisions. One of the unique advantages of the HERA $e p$ collider is that with decreasing $Q^{2}$ multiple interactions become more and more important, until eventually a situation similar to that in $p \bar{p}$ is reached when $Q^{2}=0$. Thus these effects can be studied at HERA in an experimentally controlled manner.

From the above it is clear that it is necessary to cover as large a rapidity range as possible to measure this unexplored and challenging region of QCD. We still lack a proper theoretical framework for describing and studying collective phenomena, and it is clear that without experimental investigations such as those described here we will not have the data to develop such a framework.

The rapidity range covered by HERA is shown in figure 22 together with indications of where the various effects mentioned above are expected to show up.

\subsubsection{Forward jet measurement}

The cross section for jet production in the proton direction (a forward jet) has been advocated as a particularly sensitive measure of small $x$ parton dynamics [93,94]. If the forward jet has large energy $\left(x_{\text {jet }}=E_{\text {jet }} / E_{\text {proton }} \gg x\right)$ the evolution from $x_{\text {jet }}$ to small Bjorken- $x$ can be studied. When the transverse energy of the jet is of the same order as $Q^{2}, E_{T}^{2} \sim Q^{2}$, there is no room left for $Q^{2}$ evolution and the DGLAP formalism predicts a rather small cross section in contrast to the BFKL/CCFM/LDC formalisms, which describe the evolution in $x$. 

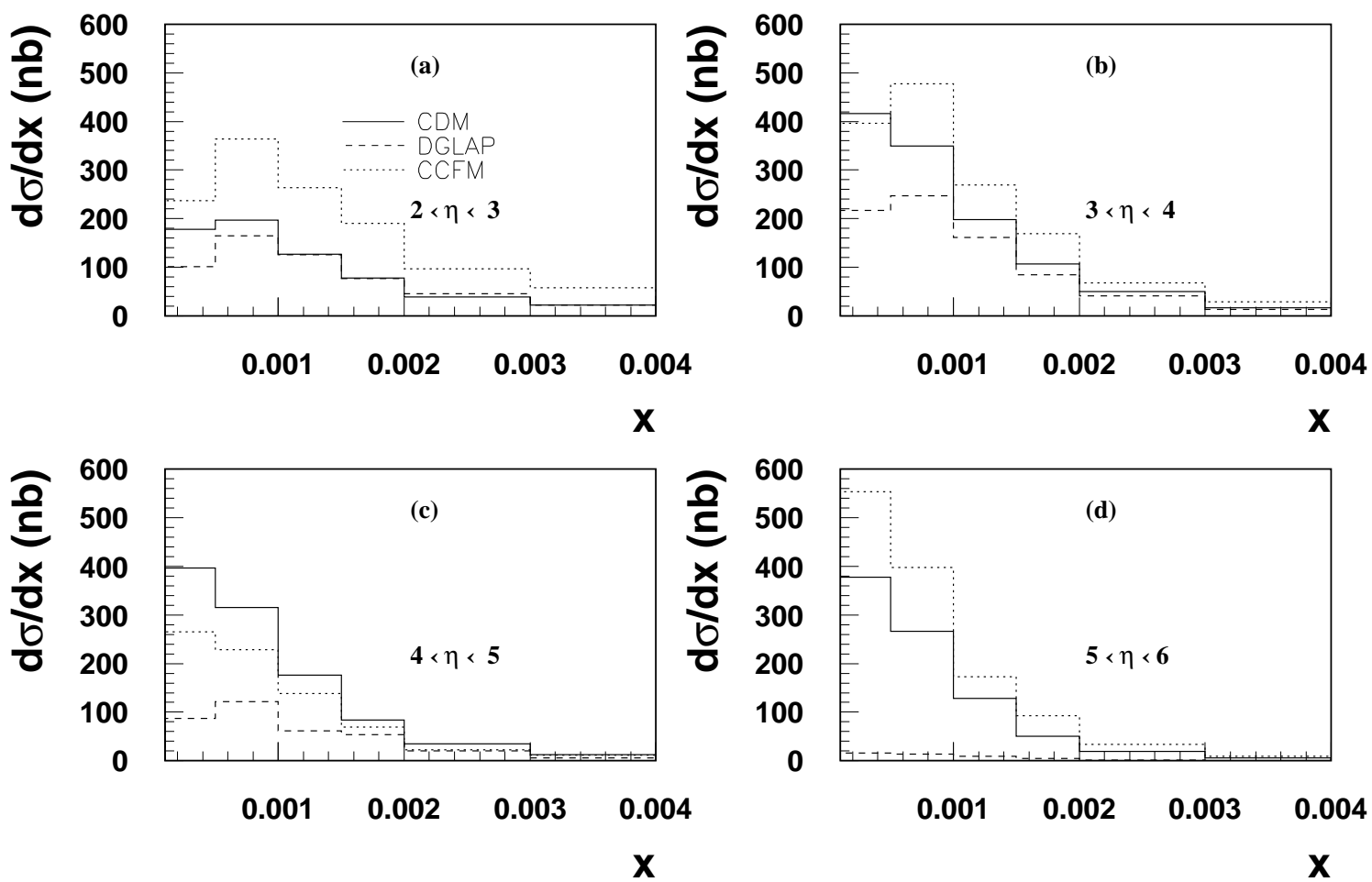

Figure 23: Forward jet cross section as a function of $x$ obtained from the CDM (ARIADNE), DGLAP (LEPTO) and CCFM (CASCADE) models. The forward jet is required to be in the angular region of $(a): 2<\eta<3,(b): 3<\eta<4,(c): 4<\eta<5,(d): 5<\eta<6$.

Measurements performed at HERA I $[95,96]$ show that the prediction from the naive DGLAP formalism lies a factor $\sim 2$ below the data, whereas the data can be described by the CCFM or LDC evolution equations equations [97], provided the non-singular terms are not included in the parton splitting functions. Description using the leading order BFKL formalism is also possible [98,99].

However, in the rapidity range covered by the present HERA experiments the forward jet cross section is also reasonably well described by the DGLAP model if a resolved virtual photon contribution is included, provided sufficient lever arm is allowed for the evolution of the resolved photon.

The measurements at HERA I were mainly restricted by two factors, the available centreof-mass energy and the geometrical acceptance of the detectors, requiring the forward jet to lie between $2 \lesssim \eta \lesssim 3$. The current high luminosity phase of HERA running, HERA II, will not allow these questions to be addressed as the focussing magnets needed to achieve high luminosities restrict the experimental acceptance in the forward and backward directions. 
In figure 23 the forward jet cross section is shown as a function of Bjorken $x$ in a larger rapidity range, up to $\eta<6$. The CDM (ARIADNE [100] ${ }^{8}$ ), a standard DGLAP (LEPTO [102]) and the CCFM (CASCADE [103]) models predict rather large cross sections, the difference with respect to DGLAP becoming more significant with increasing rapidity.

As mentioned before, the CCFM and the LDC models give a good description of the present forward jet measurements, provided only the singular terms are included in the splitting function. Approaches to include the full splitting function all give a worse description of the measurement. Is there a dynamical mechanism which suppresses the non-singular terms in the splitting functions, or increases the effective lever arm in the evolution of the resolved photon? Or is a completely new mechanism at work? Only an extended study of exclusive final state properties can provide the data that can guide us to answers to these questions.

\subsubsection{Further measurements in the forward region}

With very forward detectors one could study the transition region from diffractive to normal events. This is very important for discriminating between different models for diffraction. In pomeron or Regge based models, the leading proton or neutron is equivalent to the colliding proton by construction, whereas in soft colour interaction (SCI) type models [104] one has a small mass system which will then fragment into a leading baryon with or without accompanying hadrons. The models are now tuned to fit available data, but may perform rather differently in the as yet unobserved intermediate region. This, of course, relates to the more general problem of hadronisation and confinement.

\subsection{A 3D picture of the proton - generalised parton distributions}

Hard exclusive electroproduction processes, such as $\rho$ production or deeply virtual Compton scattering, offer the unique possibility of creating "3D" images of the nucleon. Due to QCD factorisation theorems, the information about the structure of the nucleon probed in these reactions is accumulated in process-independent matrix elements, so-called generalised parton distributions (GPD's) [105-107]. The GPD's are related to the usual parton distributions, measured in inclusive DIS, as well as to the elastic form factors of the nucleon. In particular, similar to the elastic form factors, the GPD's allow for a coordinate space interpretation in terms of a distribution of partons in the transverse plane (impact-parameter dependence) [108]. An important advantage of high-energy exclusive processes is that, in contrast to the situation with form factors, they allow the measurement of the dependence of the transverse spatial distributions on $x$ ("hadron tomography"). A measurement of these distributions would provide new insights into the non-perturbative dynamics giving rise to the quark and gluon distributions in the nucleon and would allow the resolution of many long-standing problems, such as the rôle of the "pion cloud" in the partonic structure of the nucleon, or the dynamical reason for the growth of the transverse size of the nucleon observed at small $x$. The information about the spatial distribution of partons in the transverse plane can be revealed by measuring the $t$-dependence of hard electroproduction processes [109].

\footnotetext{
${ }^{8}$ Using the tuned parameters given by "set 2 " in [101]
} 
Experimental programs dedicated to the measurement of GPD's are presently under way at JLAB (CLAS), the HERMES experiment at DESY, and COMPASS, relying mostly on deeplyvirtual Compton scattering (DVCS). The first experimental observations of DVCS were made by the $\mathrm{H} 1$ and ZEUS experiments at HERA. Measurements of DVCS and other exclusive electroproduction processes at the HERA collider would enjoy several crucial advantages over the fixed-target setup of the above experiments. First, the unique lever arm in the photon virtuality $Q^{2}$ makes possible the experimental verification that the region in which the $t$-dependence of the cross section is universal (i.e. independent of the produced diffractive state and practically $Q^{2}$-independent) has been reached, which is an essential prediction of the QCD factorisation theorem, and thus the establishment of the relevance of the hard QCD reaction mechanism [110]. Current HERA data indicate that $Q^{2} \geq 15 \mathrm{GeV}^{2}$ is needed for light meson production to reach this limit. No information is available for the DVCS case. A detailed study of the pattern of the onset of this universality will provide important information about meson wave functions and the higher-twist contributions to exclusive diffractive processes. In contrast, experiments like CLAS or HERMES have to assume the applicability of the hard QCD reaction mechanism in the analysis of their data without the possibility of independent confirmation. Moreover, the models which describe the data explicitly include large higher-twist effects [111] which also depend on $t$, making extraction of the GPD's rather problematic.

Second, the unique energy range of the HERA collider experiments would allow such measurements to be performed for values of $x$ as small as $\sim 10^{-3} \ldots 10^{-4}$. In this range, due to the dominance of the gluon distributions at small $x$, the structure of the GPD's simplifies considerably compared to the "valence" region probed in fixed-target experiments [112], facilitating the analysis of the experiments and the interpretation of the data. Furthermore, the $x$-dependence of hard electroproduction amplitudes carries interesting information about the non-perturbative dynamics giving rise to the nucleon's parton distributions. For instance, it is expected that in the hard regime the increase of the transverse size of the nucleon with decreasing $x$ is much slower than in the soft (Regge) regime. The degree to which this "slowdown" depends on $Q^{2}$ will provide unique information about the interplay of soft and hard physics.

In particular, HERA collider experiments offer a chance to measure the transverse spatial structure of the gluon distribution in the nucleon through the $t$-dependence of the $J / \psi$ and $\Upsilon$ photoproduction cross sections. Contrary to the quark distributions, whose $t$-dependence is constrained by the elastic nucleon form factors, little is known at present about the $t$-dependence of the gluon distribution (the "two-gluon form factor") of the nucleon. There are indications that the transverse size of the gluon distribution is smaller than that of the quark distributions [109]. Knowledge of the transverse spatial distribution of gluons at small $x$ will be important also for the modelling of hadron-hadron collisions at LHC energies.

The above studies require accurate measurements of the $t$-dependence of hard electroproduction cross sections. The $t$-dependence of the GPD's carries the information about the spatial distributions of partons in the transverse plane and is crucial for all attempts to reveal the "3D" structure of the nucleon. More generally, precise knowledge of the $t$-dependence of the cross sections is required to compare experimental data at finite $|t| \sim 1 \mathrm{GeV}^{2}$ with theoretical predictions at $t=0$, where they are most reliable. For example, it is known that different fits (exponential, dipole form) to the existing data for the $t$-dependence of $J / \psi$ photoproduction result in rather different extrapolations of the cross section to $t=0$, introducing a considerable uncertainty in the comparison with theoretical predictions [109]. 
The precision achievable with the H1 detector in the measurement of DVCS and hard exclusive electroproduction processes requires further investigation as does the influence of possible upgrades on the measurement of the GPDs.

\subsection{Nuclear parton distributions and saturation}

One of the most pressing and hotly discussed problems of small $x$ physics is the possible existence of a regime at small $x$ in which the parton densities are high, but the coupling constant small. In this regime, despite the small coupling constant, the interaction of small colour singlets with hadronic targets becomes comparable to the maximally possible strength of hadronic interactions for a wide range of impact parameters and approaches the geometric cross section $2 \pi R_{A}^{2}$ that pertains in the black body limit (BBL). Here, $R_{A}$ is the radius of the target nucleus.

The prime motivation for using nuclear beams at HERA is to advance much deeper into this new domain than would be possible in the electron - nucleon mode. Based on the dependence of various observables on the nucleon number, $A$, measurements at HERA would provide critical tests of various ideas about QCD in the high-density limit, including the possible saturation of gluon and quark densities [113-115] resulting in a large reduction of the quark and, especially, gluon parton densities [47] (leading twist nuclear shadowing) as compared to the incoherent sum of the nucleon densities, $g_{A}<A g_{N}$, and a large probability of coherent diffraction $(\sim$ $40 \%$ for heavy nuclei) with a strong enhancement of the diffraction to states containing charm (for a summary, see $[116,117]$ ). In addition, these studies will improve the precision of the determination of $F_{2}^{n}-F_{2}^{p}$ from the deuteron runs by testing the theory of nuclear shadowing for light nuclei, and will provide critical input for the interpretation of heavy ion collisions at RHIC and especially at the LHC, where the production of mini-jets may be strongly damped by leading twist gluon shadowing 9 .

The observation of this new regime in QCD using nuclear targets is much easier than it is in $e p$ scattering for two reasons. Firstly, heavy nuclei are much thicker than protons so that the density of partons per unit area is larger at central impact parameters by a factor of $\left(g_{A} / \pi R_{A}^{2}\right) /\left(g_{N} / \pi r_{N}^{2}\right) \approx A^{1 / 3} g_{A} / A g_{N}$, which is as large as 6 for $A=200$. If the gluon densities in the nucleus, $g_{A}$, and in the nucleon, $g_{N}$, are related by $g_{A} \approx A g_{N}$. Gaining a factor of a few is likely to be sufficient to bring the interaction close to the BBL for a large range of the resolution scale $Q^{2}$. This increase in the parton density can be expressed in terms of an effective Bjorken $x$, which is the $x$ value at which the equivalent parton density would be reached in the proton, see figure 24.

Another very important advantage of nuclei is the weak dependence of the scattering amplitude on the impact parameter for a wide range of the impact parameters (in fact, one can combine light and heavy nuclei to study the dependence of the amplitudes on the nuclear thickness). Indeed, in the nucleon case the scattering at large impact parameters is important even at very small $x$, which tends to mask the change of the regime of the interactions at small impact parameters.

\footnotetext{
${ }^{9}$ Measurements of gluon shadowing in $p A$ scattering at RHIC and the LHC will not be possible for $Q^{2} \leq 10$ $\mathrm{GeV}^{2}$.
} 

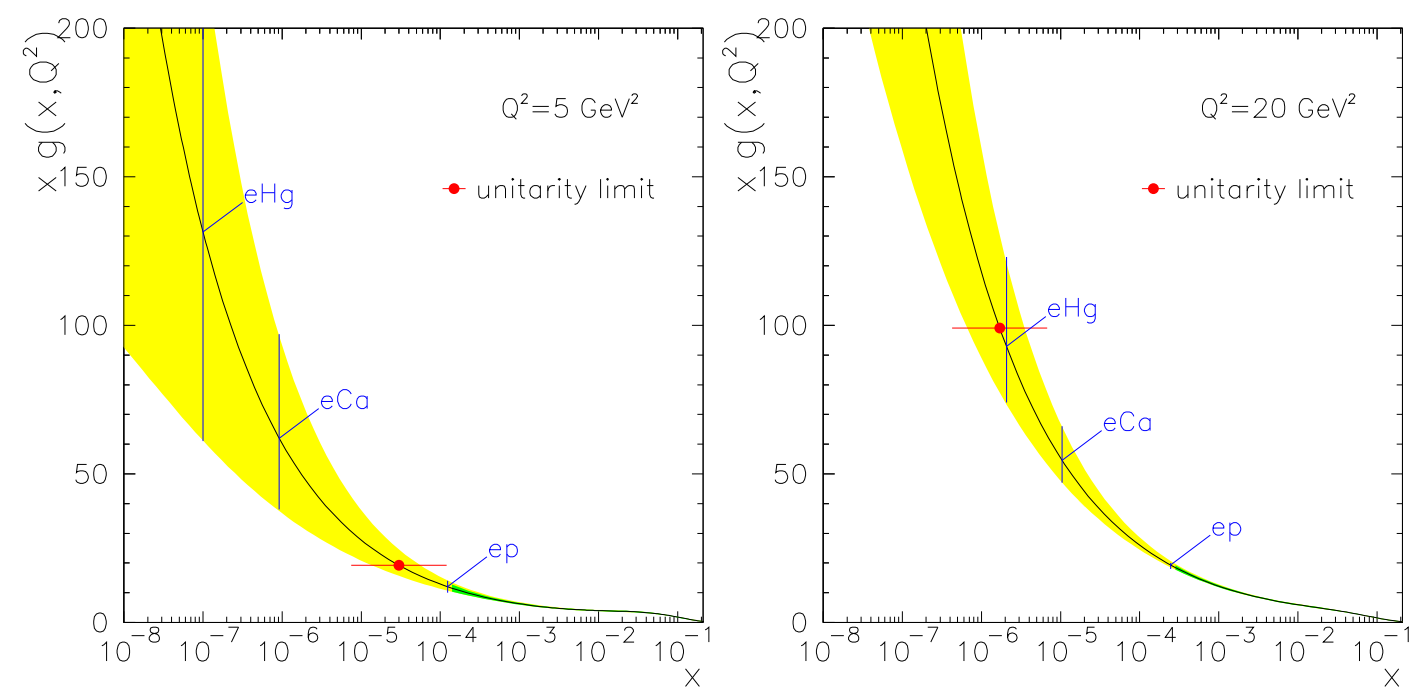

Figure 24: The gluon distribution obtained from the H1 NLO QCD fit [11] extrapolated to low values of $x$ at a $Q^{2}$ of $5 \mathrm{GeV}^{2}$ (left) and $20 \mathrm{GeV}^{2}$ (right), together with the effective Bjorken $x$ values that HERA reaches in the collision of electrons with various ion species; also indicated are the approximate limits at which saturation of the gluon distribution is expected to occur.

Up to now, deep inelastic scattering off nuclei at small $x$, where coherence effects are large, was studied only at rather small $Q^{2}$ where higher twist effects, due to e.g. diffractive vector meson production, are likely to be important. An extensive study of the HERA potential with nuclear beams was performed during the HERA workshop in 1995 and 1996 [116]. Here, we summarise some of the gold plated observables which will allow HERA to discover a number of fundamental new phenomena.

\subsubsection{Inclusive nuclear shadowing}

Fixed target data do not allow nuclear shadowing to be probed at small $x$ in DIS, where nuclear shadowing is large and dominated by the leading twist effects. There are expectations [47, 118] based on the application of the Gribov theory of nuclear shadowing [43], the Collins factorisation theorem [46] for hard diffraction and the HERA hard diffraction data, of very significant shadowing for all parton densities both for light and heavy nuclei, see figure 25 .

The uncertainties in the predictions in figure 25 related to the input diffractive parton densities are smallest for the $F_{2}^{A}$ case. The current experimental errors for $F_{2}^{D(4)}\left(x_{\mathbb{P}}, \beta, Q^{2}, t \sim 0\right)$ are $\sim 15 \%$ and lead to similar or smaller uncertainties for the value of $1-F_{2}^{A} /\left(A F_{2}^{N}\right)$. In the gluon case, the uncertainties are much larger since the slope of the gluon-induced diffraction has yet to be measured. The resulting range for gluon shadowing within the model of reference [118] is given by the solid curves in figure 26. The curves were obtained using the gluon parton distribution from the $1997 \mathrm{H} 1$ diffractive fit and with the gluon distribution multiplied by the factor 0.75 (this mimics the new $2002 \mathrm{H} 1$ fit for the gluons). While the latter scenario results in lower nuclear shadowing for the gluon nuclear density for $x>7 \times 10^{-5}$, the difference between the two scenarios for the gluon shadowing does not affect the predictions for $F_{2}^{A} /\left(A F_{2}^{N}\right)$ 

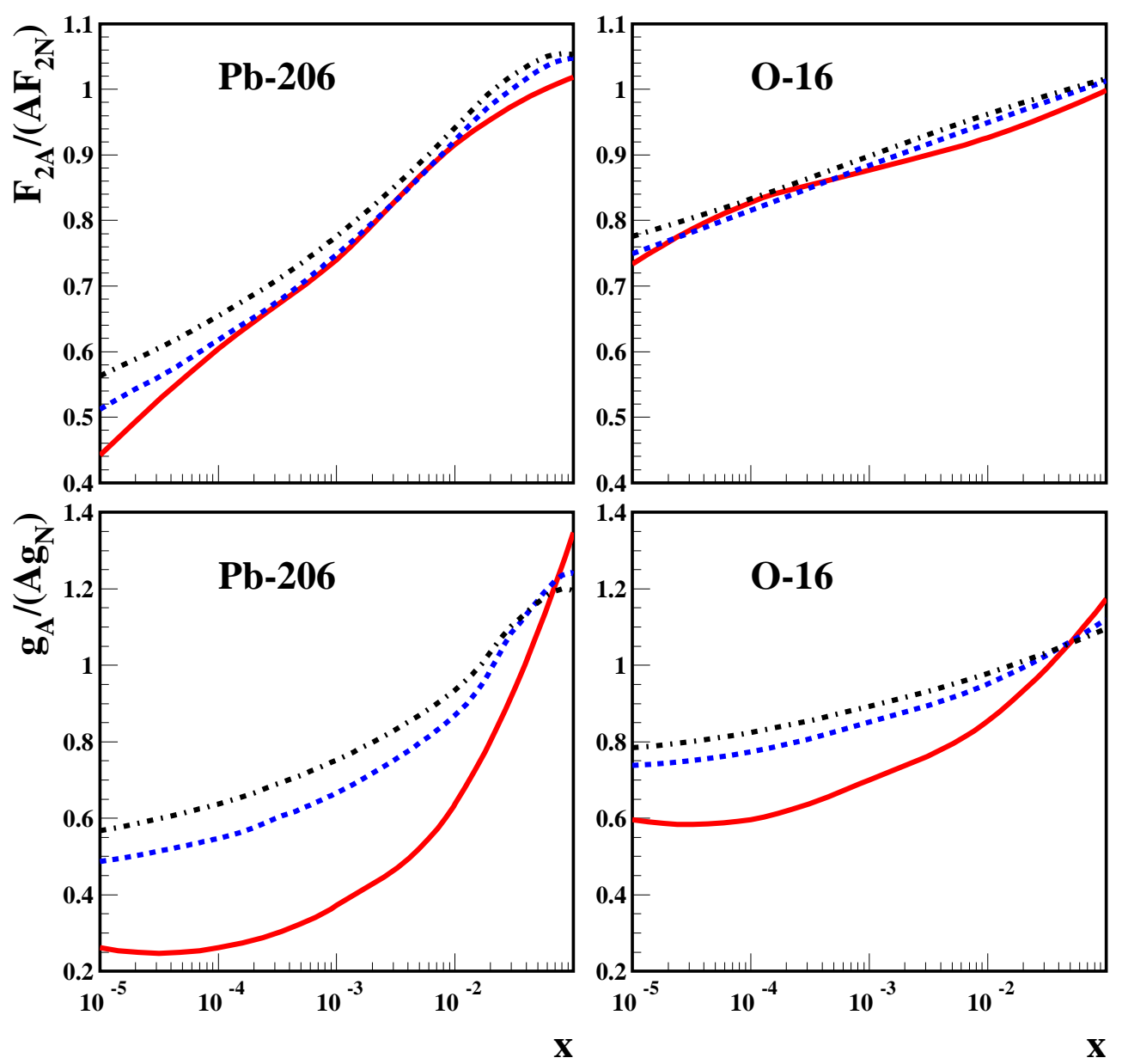

Figure 25: Predictions of the leading twist theory of nuclear shadowing [118] for the ratios $F_{2}^{A} /\left(Z F_{2}^{p}+N F_{2}^{n}\right)$ and $g_{A} /\left(A g_{N}\right)$ for ${ }^{16} \mathrm{O}$ and ${ }^{206} \mathrm{~Pb}$ as a function of $x$. The solid curve corresponds to $Q=2 \mathrm{GeV}$; the dashed curve to $Q=5 \mathrm{GeV}$ and the dash-dotted curve to $Q=10 \mathrm{GeV}$. 

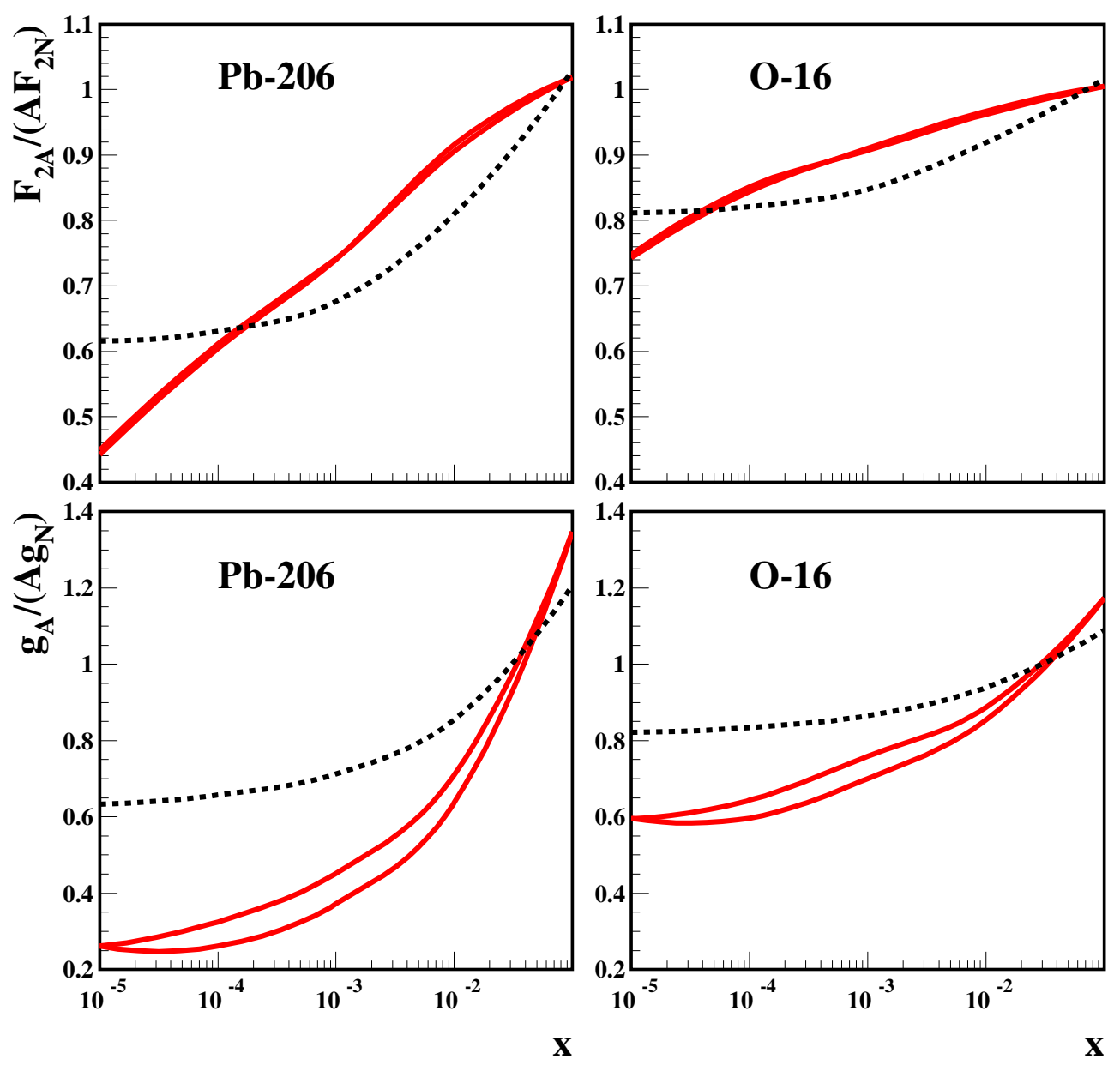

Figure 26: Nuclear shadowing for the ratios $F_{2}^{A} /\left(Z F_{2}^{p}+N F_{2}^{n}\right)$ and $g_{A} /\left(A g_{N}\right)$ for ${ }^{16} \mathrm{O}$ and ${ }^{206} \mathrm{~Pb}$ : the predictions of reference [118] (solid curves) are compared to those of reference [119] (dashed curves). All curves correspond to $Q=2 \mathrm{GeV}$.

(the two curves are virtually indistinguishable). In figure 26, we also show the results of the model of Eskola et al. [119] (dashed curves) for ${ }^{16} \mathrm{O}$ and ${ }^{206} \mathrm{~Pb}$ and at $Q=2 \mathrm{GeV}$. This model is based on fits to the limited available DIS nuclear data, assuming dominance of the leading twist and also predicts large nuclear shadowing effects. If the effects are as large as shown in figures 25 and 26, they will easily be observed in $F_{2}$ measurements at HERA.

Due to the large parton densities, the $Q^{2}$ evolution of $R_{A}=F_{2}^{A} / F_{2}^{d}$ at a $Q^{2}$ of a few $\mathrm{GeV}^{2}$ may violate the leading twist expectations. Hence the measurements of the scaling violations of $R_{A}$ (which can be done at HERA with precision $\sim 1 \%$ [116]) may provide a clean signal of the onset of the regime of nonlinear dynamics. At larger $Q^{2}$, these measurements will provide one of the ways of measuring gluon shadowing. Other options for the measurement of gluon shadowing include high $p_{t}$ jet production, $J / \psi$ production, etc.

Thus, inclusive measurements at HERA will make possible the discovery of leading twist 
shadowing and sensitive searches for a new high density/BBL regime in DIS. The predictions of the BBL for DIS are drastically different from the scaling limit: $F_{2}^{A} \propto 2 \pi R_{A}^{2} Q^{2} \ln (1 / x)$ for $Q^{2} \leq Q_{\mathrm{bbl}}^{2}(x)$, where $Q_{\mathrm{bbl}}^{2}(x)$ denotes the maximal value of $Q^{2}$ for which the total inelastic cross section of the interaction of a small colour dipole of transverse size $\propto 1 / Q$ with a heavy nucleus is equal to the black body limit, $\pi R_{A}^{2}$. The scale $Q_{\mathrm{bbl}}^{2}(x)$ increases with $A$. The measurement of the scaling violations of $F_{2}^{A}\left(x, Q^{2}\right)$ provides direct access to the dynamics of the interaction of small dipoles with nuclei and yields detailed information on the relevance of the black body limit for $\gamma^{*} A$ scattering. Direct tests of the onset of the black body regime in the gluon channel will be possible via the measurement of $\sigma_{L}(e A)$ and the study of " $2+1$ " jet events [116].

\subsubsection{Inclusive hadron observables}

A number of measurements of inclusive single hadron production would make possible the exploration of the kinematic limit at which the leading twist (LT) approximation breaks down. Two of the simplest signals are related to the basic implications of LT QCD factorisation for leading hadron production. One signal is the decrease of the ratio of leading hadron multiplicity $N(z)$ with $A$ at $z \geq 0.1$, see reference [120]. Another is the increase of the average $p_{t}$ of the partons (and hence of the produced hadrons) with $A$ due to the increase of the saturation scale with the increase of the density: $\left\langle p_{t}^{2}\right\rangle \sim Q_{\mathrm{bbl}}^{2}$ [114]. This gross violation of the QCD factorisation theorem for leading hadron production in DIS provides one of the model-independent signals of the breakdown of the LT approximation.

\subsubsection{Diffractive observables}

Observation of coherent diffraction in scattering off nuclei does not pose difficulties beyond those of the $e p$ case. Indeed, the situation may even be simpler (especially in the case of heavy nuclei) since the majority of the diffractive interactions leave the nucleus intact. At the same time, in inelastic processes nuclear breakup is accompanied by the emission of several protons and neutrons at small angles which may be used as a veto for incoherent nuclear diffraction, since the neutrons produced at zero angle $\left(\theta \leq 2 \times 10^{-4} \mathrm{rad}\right)$ can be detected by the forward neutron calorimeter.

The measurement of inclusive deep inelastic diffraction provides a test of how closely the black body limit is being approached. In this limit the probability of coherent diffraction is close to $50 \%$ of the total cross section [43,121-123]. The same is true for partial cross sections such as for charm or dijet production in $\gamma A$ scattering. Moreover, since the interaction is stronger in the gluon channel, the diffractive cross section should be close to $50 \%$ of the corresponding total cross section in a wider $\left(x, Q^{2}\right)$ range [47]. The differential cross section for diffractive production of states with mass $M_{X}^{2} \leq Q_{\mathrm{bbl}}^{2}$ is also predicted in a model-independent way [120] to be

$$
\frac{d F_{T}^{D(2)}\left(x, Q^{2}\right)}{d M_{X}^{2}}=\frac{\pi R_{A}^{2}}{12 \pi^{3}} \frac{Q^{2} M_{X}^{2} \rho\left(M_{X}^{2}\right)}{\left(M_{X}^{2}+Q^{2}\right)^{2}},
$$

where $\rho\left(M_{X}^{2}\right)=\sigma^{e^{+} e^{-} \rightarrow \text { hadrons }}\left(s=M_{X}^{2}\right) / \sigma^{e^{+} e^{-} \rightarrow \mu^{+} \mu^{-}}\left(s=M_{X}^{2}\right)$. 
Another signature of the black body limit is that, for $M_{X}^{2} \leq Q_{\mathrm{bbl}}^{2}$, the production of high- $p_{t}$ jets is strongly enhanced: $\left\langle\left(p_{t}^{\text {jet }}\right)^{2}\right\rangle=3 M_{X}^{2} / 20$. One especially sensitive test of the violation of the LT approximation is exclusive dijet production by quasi-real photons [124]. In the LT approximation, this process is strongly suppressed [125], while it constitutes the dominant contribution to diffraction in the BBL. Hence, studies of this process make it possible to distinguish between the suppression of the total cross section due to leading twist shadowing and the suppression due to the proximity to the BBL.

\subsubsection{Exclusive deep inelastic diffraction}

The production of vector mesons in the process $\gamma^{*}+A \rightarrow V+A$, is simple to separate from incoherent events (for this events in which the detector has acceptance for vector mesons produced in the direction of the virtual photon) due to the very steep diffractive peak. This makes possible the investigation of the fundamental question: are heavy nuclei transparent for high-energy small objects like $J / \psi$ or $\Upsilon$ mesons? In the region of $x \sim 0.02$, evidence for colour transparency was obtained [126] by the observation that the coherent $J / \psi$ production amplitude is proportional to $A F_{A}(t)$, where $F_{A}(t)$ is the nuclear form factor. This colour transparency regime corresponds to the propagation of a small dipole through a thick target with very small absorption. At small $x \leq 0.01$, a qualitatively new phenomenon - colour opacity - is expected: the strong absorption of small dipoles propagating through the nuclei. In the black body limit the increase of the cross section with $A$ will be reduced to $A^{2 / 3}$ as compared to $A^{4 / 3}$ in the colour transparency limit. QCD also predicts the absolute cross section for vector meson production in the black body limit. At HERA one would be able to study both intermediate $x \sim 0.01$ kinematics, where colour transparency is expected at large $Q^{2}$ for light mesons and at all $Q^{2}$ for the production of onium states, and also investigate the region of colour opacity in the regime where LT dominates and the suppression of the cross section is given by the square of $g_{A} / g_{N}$, which rather weakly depends on $Q^{2}$ for $Q^{2} \geq 20 \mathrm{GeV}^{2}$ (c.f. figure 25). In contrast, the eikonal models of the dipole-nucleus interaction [113] predict that corrections to colour transparency die out as $1 / Q^{4}\left(\propto 1 / m_{Q}^{4}\right.$ for onium production). This can also be studied in the region where one can probe the approach to the BBL.

A unique feature of exclusive vector meson production is that it can be used to check the proximity to the BBL even for intermediate $Q^{2}$, since the cross section for the production of vector mesons in the BBL can be calculated in a model-independent way [120]:

$$
\frac{d \sigma_{T}^{\gamma_{T}^{*}+A \rightarrow V+A}}{d t}=\frac{M_{V}^{2}}{Q^{2}} \frac{d \sigma_{L}^{\gamma_{L}^{*}+A \rightarrow V+A}}{d t}=\frac{\left(2 \pi R_{A}^{2}\right)^{2}}{16 \pi} \frac{3 \Gamma_{V} M_{V}^{3}}{\alpha\left(M_{V}^{2}+Q^{2}\right)^{2}} \frac{4\left|J_{1}\left(\sqrt{-t} R_{A}\right)\right|^{2}}{-t R_{A}^{2}}
$$

where $\Gamma_{V}$ is the electronic decay width for the process $V \rightarrow e^{+} e^{-}$. Equation (19) predicts the $Q^{2}$-dependence of the cross section which is distinctively different from the LT prediction. The $Q^{2}$-dependence of equation (19) is a factor $\propto\left(1+Q^{2} / m_{V}^{2}\right)^{-2}$ weaker than that in the LT limit [120].

To summarise, the use of nuclear beams would allow a major increase in the potential of HERA for the study of the dynamics of QCD at high densities and in the search for nonlinear QCD phenomena. Several phenomena, especially in the diffractive channels, will allow the 
unambiguous investigation of whether the black body regime is reached and the detailed exploration of the new state of QCD matter produced at small $x$. Most of these measurements will require rather modest luminosities, $1-10 \mathrm{pb}^{-1}$ per nucleus [116]. It would be possible to explore the nonlinear regime over at least a factor two larger range in $Q^{2}$ by performing experiments using a series of nuclei: $A=2,4,16,40$ and even further, if heavy ions are added to the programme.

\section{Mapping nucleon and photon spin}

\subsection{Measuring the spin structure of longitudinally polarised nucleons}

Nucleon spin presents a fascinating puzzle, the detailed study of which was initiated by the EMC [127]. The EMC observed that the quark contribution to spin is surprisingly small and hence that nucleon spin cannot be understood within the naive quark parton model. Since then a wealth of data on spin structure has been accumulated from fixed-target experiments and spin theory has become much more sophisticated. The puzzling question of the composition of nucleon spin is, however, still unresolved (for a summary of the present status see [128]). The importance of extending the kinematic range of spin physics with HERA has been investigated and emphasised in a series of workshops on polarised ep physics at HERA [129].

In polarised DIS, the spin-dependent terms only make a small contribution to the total cross section. They can be extracted from measurements of cross section differences for interactions with opposite relative orientations of lepton and nucleon helicities, in which the spinindependent contributions cancel. A classic quantity is the spin structure function $g_{1}$, which measures the weighted sum of polarised quark distribution functions $\Delta q$ and is approximately related to the cross section asymmetry, $A_{\|}=\left(\sigma_{\uparrow \downarrow}-\sigma_{\uparrow \uparrow}\right) /\left(\sigma_{\uparrow \downarrow}+\sigma_{\uparrow \uparrow}\right)$, by:

$$
g_{1} \simeq \frac{F_{2}}{2 x} \cdot \frac{A_{\|}}{\lambda_{e} \lambda_{p}} \cdot \frac{y^{2}+2(1-y)}{y(2-y)}
$$

Equation 20 illustrates the need for high polarisations, $\lambda_{e}$ and $\lambda_{p}$, and a preference for measurements at large values of $y$. Polarisations $\lambda_{p} \simeq 0.6$ may be achieved in the HERA proton ring. From equation 20 it can be deduced that large integrated luminosities are necessary for accurate studies of the proton spin structure.

The measurement of small asymmetries at low $x$ has been studied previously [130], and detector effects have been found to be small. Event migration rates and radiative corrections can be controlled by the use of hadronic event reconstruction methods. The use of these also gives access to asymmetries at high $x$, where the polarisation of the virtual photon is as low as 0.03 , a kinematic range which is barely accessible in fixed target experiments.

For this Letter of Intent, simulations were performed of both the asymmetries that must be measured in polarised $e p$ and $e d$ scattering (figures 27 and 28, respectively) and the $g_{1}$ structure functions that can then be extracted (figures 29 and 30). The simulations are compared with recent theoretical calculations [131, 132], one of which [133] includes the uncertainties of the predictions. The asymmetries are large at large $x$ and will be measurable with luminosities of the 

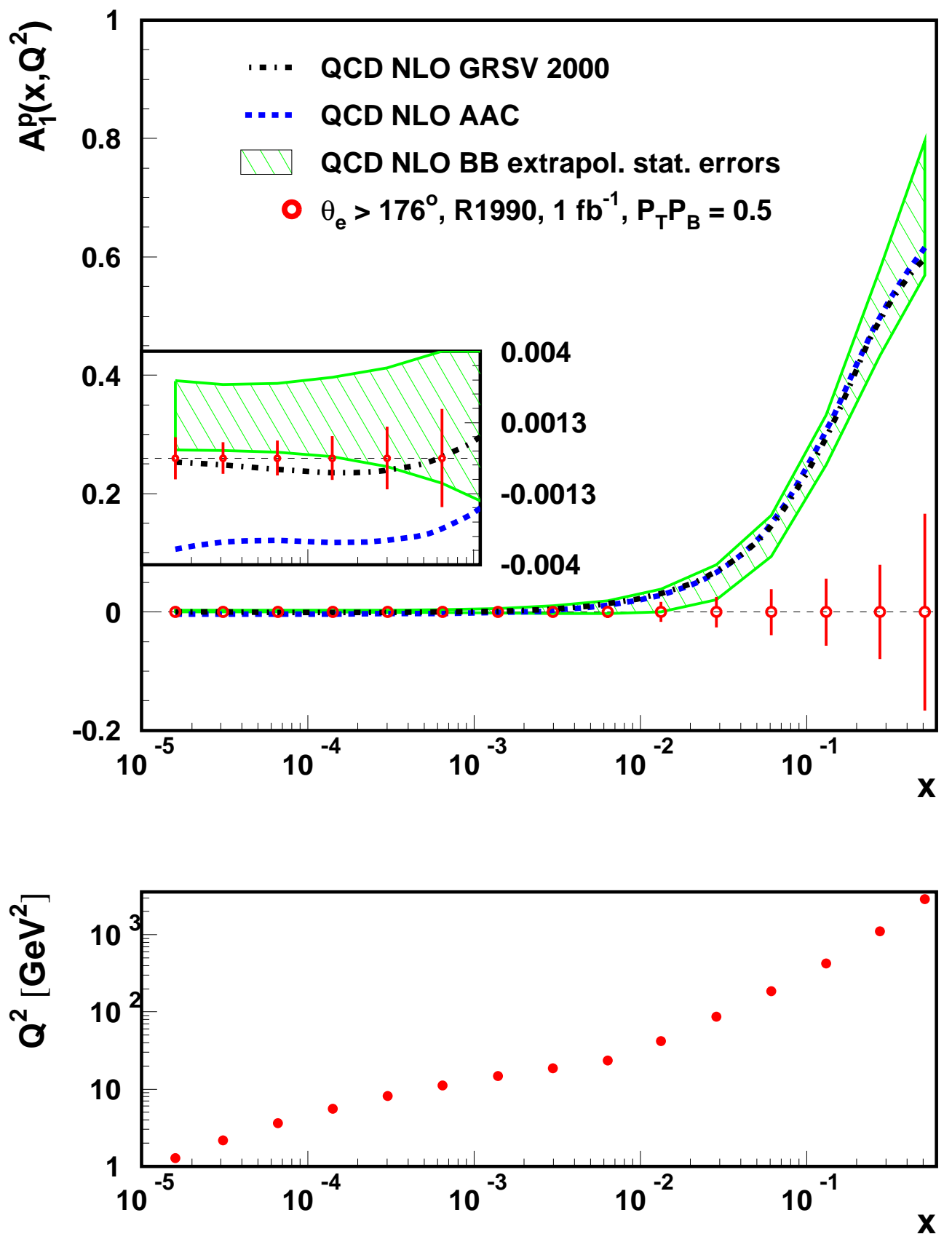

Figure 27: Simulated measurement of the asymmetry $A_{1}^{p}$ at HERA for conditions as specified in the legend. Note that the measurement of the asymmetry versus $x$ is made at an approximately constant value of $y$, i.e. $Q^{2}$ rises with $x$, as shown in the lower plot, such that this measurement is exploring a completely new kinematic domain. At high $x$ the QCD evolution of spin is tested, which fixes $\alpha_{s}$ much more accurately than before, while the emphasis at low $x$ is on the unknown $x$ dependence of the cross section. 

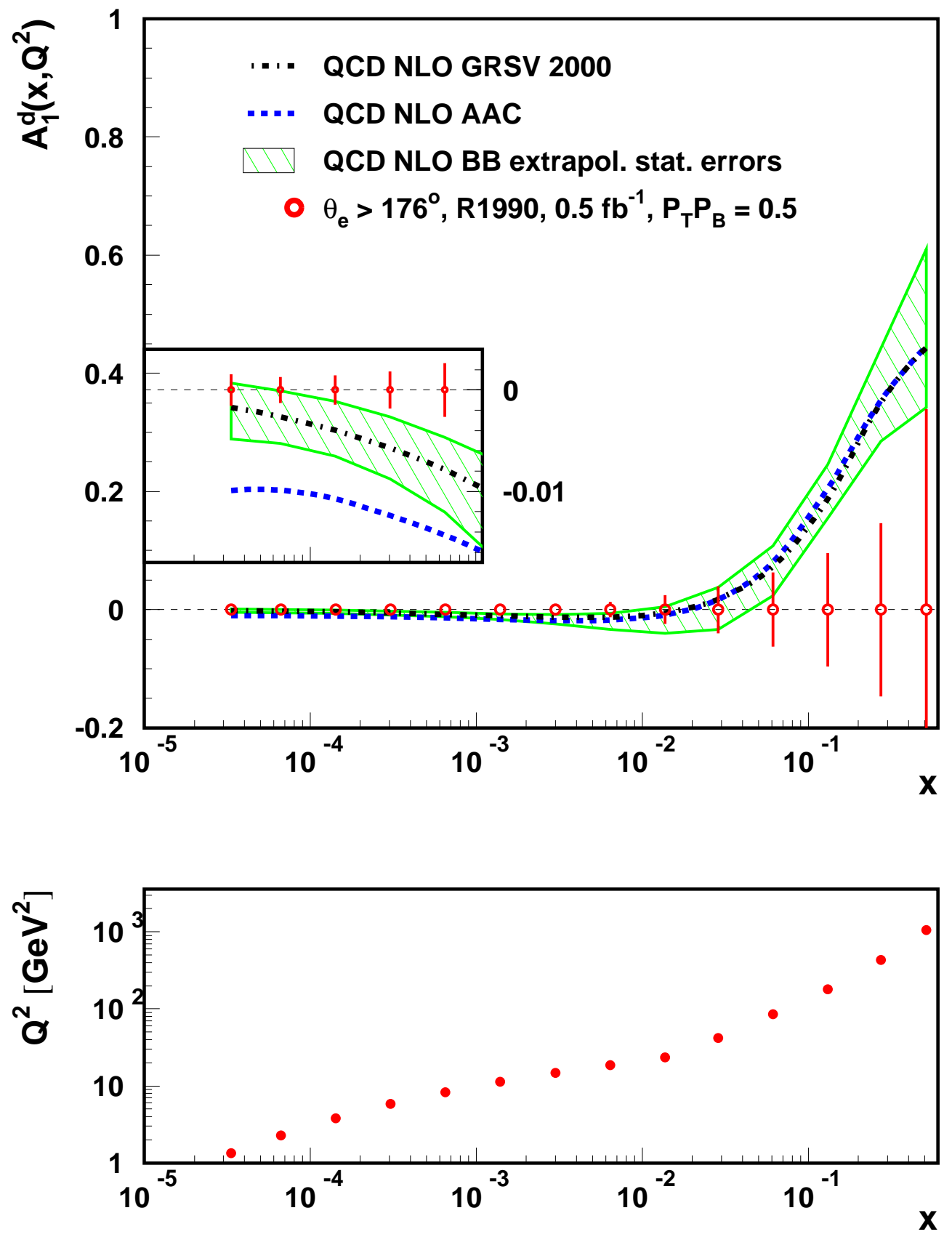

Figure 28: Simulated measurement of the asymmetry $A_{1}^{d}$ at HERA for conditions as specified in the legend. Because of the smaller anomalous magnetic moment, depolarising resonances are 24 times less frequent for a polarised deuteron beam than for polarised protons which may make this asymmetry measurement easier than that for protons. 
order of $100 \mathrm{pb}^{-1}$ per polarisation state, both in $e p$ and in $e d$ scattering. While the asymmetries at low $x$ are small, closer inspection reveals interesting differences from naive expectations. For example, the BB fit predicts a $1 \%$ asymmetry at $x=0.001$ and $Q^{2}=10 \mathrm{GeV}^{2}$. The predictions for $g_{1}$ vary remarkably. As was the case for unpolarised DIS at low $x$, exploratory measurements can be performed with $50 \ldots 100 \mathrm{pb}^{-1}$ while detailed investigations will require significantly larger luminosities. A programme of polarised low $x$ physics at HERA would make possible a unique and ground breaking series of measurements.

The outstanding advantage of HERA over the high-statistics fixed target experiments COMPASS and HERMES and at SLAC is the large extension of the kinematic range. The study of spin-dependent effects can be extended to low $x$, making possible a test of the $Q^{2}$ evolution of $g_{1}$ up to $Q^{2} \simeq 10^{3} \mathrm{GeV}^{2}$. Information on the spin structure can also be obtained from data on asymmetries in the production of jet or hadron pairs. A completely new field is diffraction in polarised scattering, see [136]. The polarised gluon distribution $\Delta G$ can be measured using dijet events, as was demonstrated in [134]. A further source of information about gluon polarisation at low $x$ is charm production, which in unpolarised scattering at HERA represents roughly $20 \%$ of the inclusive cross section.

\subsection{Polarised electron-deuteron scattering and shadowing effects}

In the case of polarised deuteron scattering, the situation with respect to shadowing appears to be favourable for the inclusive measurements of $g_{1}^{n}$ at small $x$. Indeed, experimentally, for in small $x$ range covered by the fixed target experiments, $g_{1}^{n} \ll g_{1}^{p}$. The nuclear shadowing correction is multiplicative and hence modifies $g_{1}^{d}$ more strongly than $F_{2}^{d}$ (if soft dynamics dominates at the normalisation scale, the shadowing effect in the spin case could be two times larger, see reference $[137,138]$ ), and may lead to corrections to the asymmetry of the magnitude of the value of the shadowing correction for $F_{2}^{d}$, that is a few per cent. Hence it would lead to an even smaller correction for the determination of $g_{1}^{n}$. One can also use tagging of the scattering off the neutron via detection of leading protons. The same comments concerning shadowing corrections are applicable in this case. In addition, the detection of the spectator proton allows the elimination of the depolarisation factor of $3 P_{D} / 2 \sim 0.1\left(P_{D}\right.$ is the probability that the deuteron is in a $D$-wave state) and also allows the measurement of the deuteron polarisation, since the longitudinal spectrum of protons is sensitive to the polarisation of the deuteron. This is also the case in unpolarised electron-deuteron scattering, see the detailed discussion in [139].

Note also that the experiments with deuteron beams can be used to explore the effects of colour transparency and colour opacity via the study of coherent processes with the deuteron, for example production of vector mesons in the reaction $\gamma^{*}+d \rightarrow V+d$ at large enough $t$. The use of the polarised deuteron will be especially beneficial for the study of these effects [59].

\subsection{Charged current interactions and flavour dependent spin structure}

Inclusive measurements in DIS are sensitive to the sum of all quark flavours. To extract flavourdependent spin information, one presently uses semi-inclusive scattering, an area being actively pursued in fixed-target experiments. In the high $Q^{2}$ range of HERA, however, CC interactions 

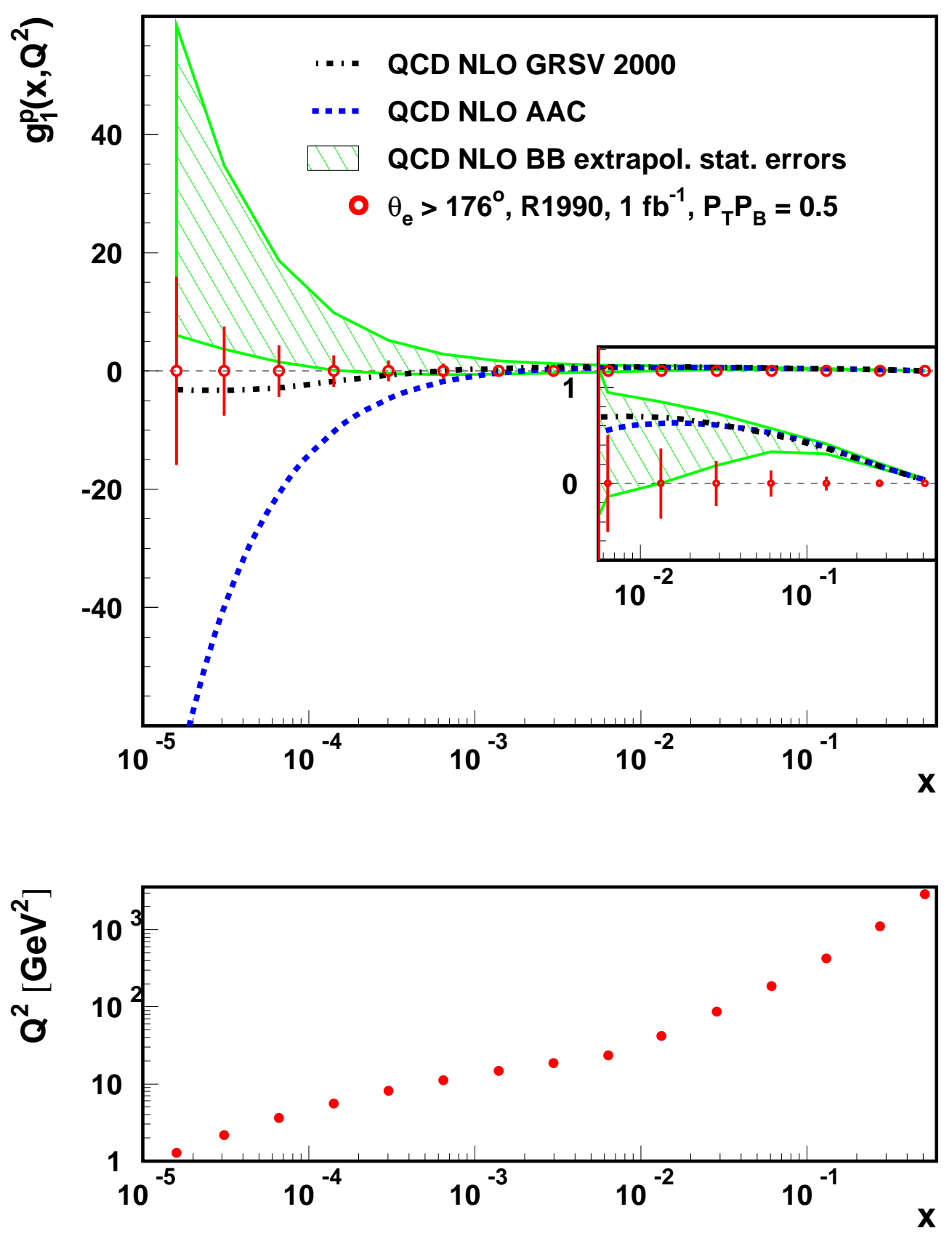

Figure 29: Simulated measurement of the polarised structure function $g_{1}^{p}$ at HERA for conditions as specified in the legend. The curves denote recent theoretical predictions and extrapolations. Clarification of the behaviour of $g_{1}$ at low $x$ is one of the fundamental tasks of polarised DIS at HERA. 

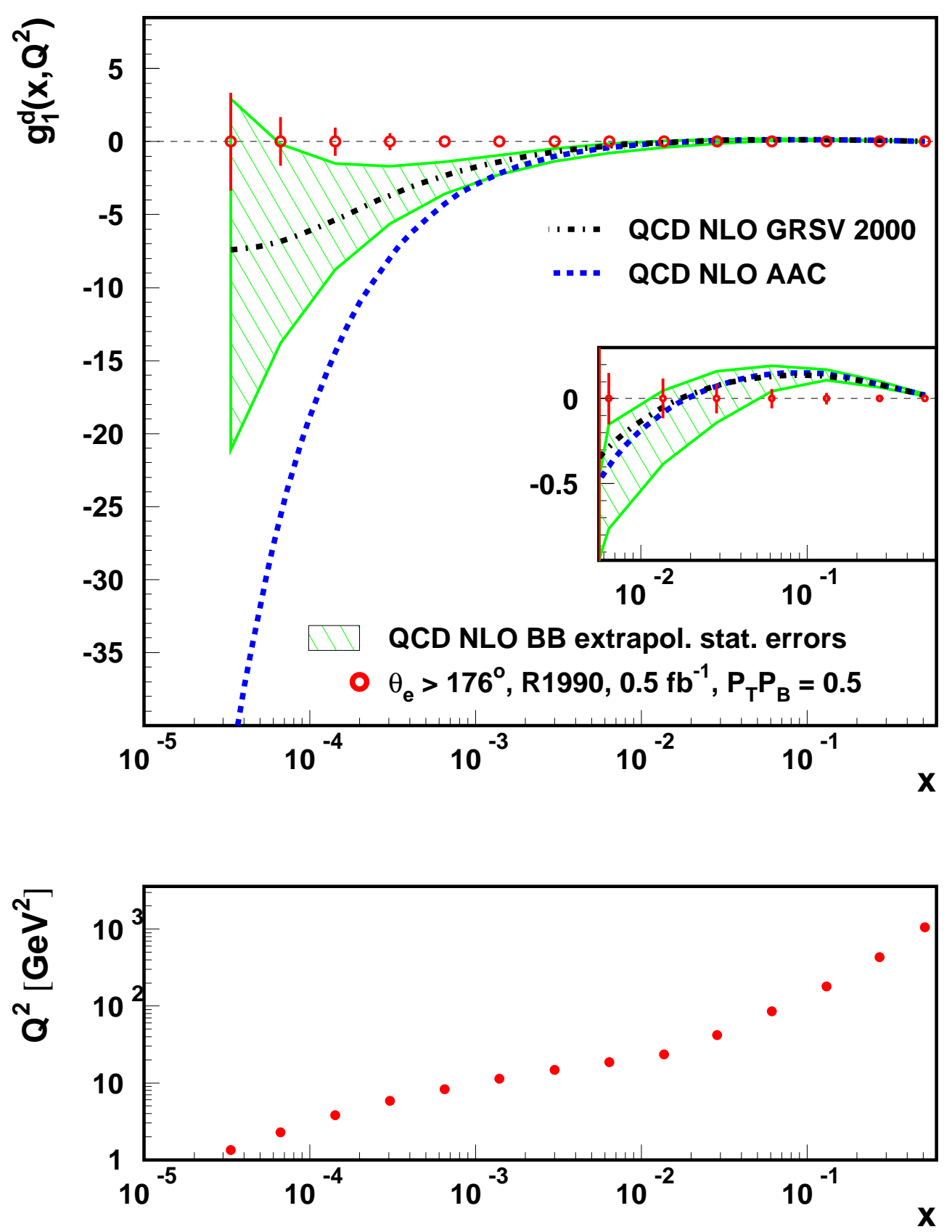

Figure 30: Simulated measurement of the polarised structure function $g_{1}^{d}$ at HERA for conditions as specified in the legend. The curves denote recent theoretical predictions and extrapolations which differ markedly at low $x$. At larger $x$ the evolution as prescribed by pQCD will be tested, since the largest $x$ data correspond to $Q^{2}$ values of about $1000 \mathrm{GeV}^{2}$, values at which spin physics has not yet been studied. 
provide access to flavour-specific spin information, independent of fragmentation effects which hinder semi-inclusive analyses. In the $\mathrm{CC} e^{+} p$ and $e^{-} p$ scattering cross sections, the following asymmetries can be defined:

$$
A^{W^{\mp}}=\frac{d \sigma_{\uparrow \downarrow}^{W^{\mp}}-d \sigma_{\uparrow \uparrow}^{W^{\mp}}}{d \sigma_{\uparrow \downarrow}^{W^{\mp}}+d \sigma_{\uparrow \uparrow}^{W^{\mp}}}=\frac{ \pm 2 b g_{1}^{W^{\mp}}+a g_{5}^{W^{\mp}}}{a F_{1}^{W^{\mp}} \pm b F_{3}^{W^{\mp}}} \approx \frac{g_{5}^{W^{\mp}}}{F_{1}^{W^{\mp}}}
$$

with $a=2\left(y^{2}-2 y+2\right)$ and $b=y(2-y), g_{5}^{W^{-}}=\Delta u+\Delta c-\Delta d-\Delta s$ and $g_{5}^{W^{+}}=$ $\Delta d+\Delta s-\Delta u-\Delta c$. A study of the measurement of this asymmetry, requiring the total missing transverse momentum to exceed $12 \mathrm{GeV}$, has demonstrated that there is sensitivity to $g_{5}$ [140]. The $g_{5}$ structure functions are related by a sum rule due to Bjorken, which is valid for very large $Q^{2}[141]$.

\subsection{Physics with transverse nucleon polarisation}

The study of deep inelastic charged lepton scattering off transversely polarised protons or deuterons is important if the picture of the internal spin structure of the nucleon is to be completed. While the polarised structure function $g_{2}$ is kinematically suppressed by a factor $\left.m_{p} x / \sqrt{(} Q^{2}\right)$, and thus only accessible in very high statistics experiments, the determination of the still unknown third type of twist-two quark distribution function $\delta q$ or $h_{1}(x)$, termed transversity and introduced in [142], seems to be feasible with polarised HERA running, since $h_{1}$ is expected to be of similar size to $g_{1}$ [143].

In order to probe the transverse spin polarisation of the nucleon, a helicity (identical to chirality at leading twist) flip of the struck quark must occur. In hard processes this is only possible with non-zero quark masses, hence the suppression of this function in inclusive DIS. However, in semi-inclusive processes this can occur via the combination of two chiral odd functions, one describing the quark content of the target $(\delta q)$ and the other describing the fragmentation of the quark into hadrons. Considerable effort has gone into understanding, modelling and elucidating possible measurements of $\delta q$. A review is given in [144].

Presently, two fixed target experiments, HERMES at DESY and COMPASS at CERN, are running with transversely polarised targets with the goal of studying transversity distributions and chiral-odd fragmentation functions. But in neutral current DIS the Collins ( $\mathrm{T}$ and chiral odd, sensitive to $h_{1}$ ) and Sievers (T-odd, not sensitive to $h_{1}$ ) effects are expected to contribute to the single spin semi-inclusive asymmetries measured with a transversely polarised nucleon.

Here, a measurement of e.g. semi-inclusive pion production at very high centre-of-mass energies of the photon-nucleon-system, $W$, would open a completely new kinematic range for transversity measurements, where a little is known [145,148]. For example, charged currents deliver a unique opportunity to further disentangle the two competing processes contributing to single spin semi-inclusive asymmetries in neutral current DIS, since in charged current cross sections no chiral-odd functions occur, i.e. only the so called Sievers effect contributes. Furthermore, at a polarised collider, the current and target fragmentation regions can be much more cleanly distinguished, making a better theoretical description possible [149].

Semi-inclusive measurements using a transversely polarised nucleon beam at HERA give access to a broad spectrum of fundamental spin-dependent quantities, allowing both new studies and independent checks of polarisation measurements performed so far only by fixed target experiments. 


\subsection{Photon spin}

Nothing is known experimentally about the parton content of circularly polarised photons. While the current round of spin experiments, COMPASS and RHIC, is not sensitive to the polarised parton distributions of the photon, the photoproduction of jets at HERA with polarised beams gives access to these distributions. Only limited luminosities of $\mathcal{O}\left(50 \mathrm{pb}^{-1}\right)$ are necessary for the measurements [146, 147].

\subsection{Summary}

It is evident that polarised $e N$ physics at HERA is a rich field about which little is known from current experiments, all of which operate in a much more confined kinematic domain. HERA, with its large centre-of-mass energy and hence lever arm in $Q^{2}$ and $x$ will be able to pin down the polarised gluon distribution. With the further possibility of measuring generalised parton distributions, HERA could play a major rôle in clarifying the spin puzzle. Further, if any deviation from the Standard Model is found, such as $R$-parity violating SUSY, leptoquarks or instantons, it will be particularly interesting to study the helicity-specific properties of the corresponding objects in $\vec{e} \vec{p}$ or $\vec{e} \vec{d}$ scattering at HERA. The luminosities required are in excess of 50 to $100 \mathrm{pb}^{-1}$ per beam polarisation state. This is achievable following the HERA luminosity upgrade if machine developments for polarised deuterons and/or protons are pursued.

\section{Upgrades to the $\mathrm{H1}$ apparatus}

\subsection{Introduction}

This section describes briefly the upgrades to the $\mathrm{H} 1$ detector that are necessary for the physics programme described above. We consider first the addition of a proton spectator tagging system downstream of $\mathrm{H} 1$ in the proton direction. This is the only upgrade that is essential for the electron-deuteron programme described in section 2. The tagging system could be added to $\mathrm{H} 1$ with the current HERA II optics, but it could equally well be used with the HERA I optics, i.e. following the removal of the superconducting GO and GG magnets. The former configuration has the advantage of higher luminosity, the latter that the beam dispersion at the interaction point is smaller, and hence that measurements of $|t|$ can be made with higher precision in diffractive processes through the detection of the $p_{t}$ of the scattered nucleon.

The other upgrades discussed, to the forward and backward regions of the experiment, can only be performed if the GG and GO magnets are removed, i.e. the low luminosity HERA I optics are restored. In these cases, the measurements for which the upgrades are designed do not require high luminosity; it is the improved acceptance in the forward and backward regions which is critical to their success.

It is as yet too early to decide how and when the upgrades suggested here should be carried out. This will depend on the situation with regard to future HERA running. For example, if running with deuterons were to take place immediately after the completion of the current 


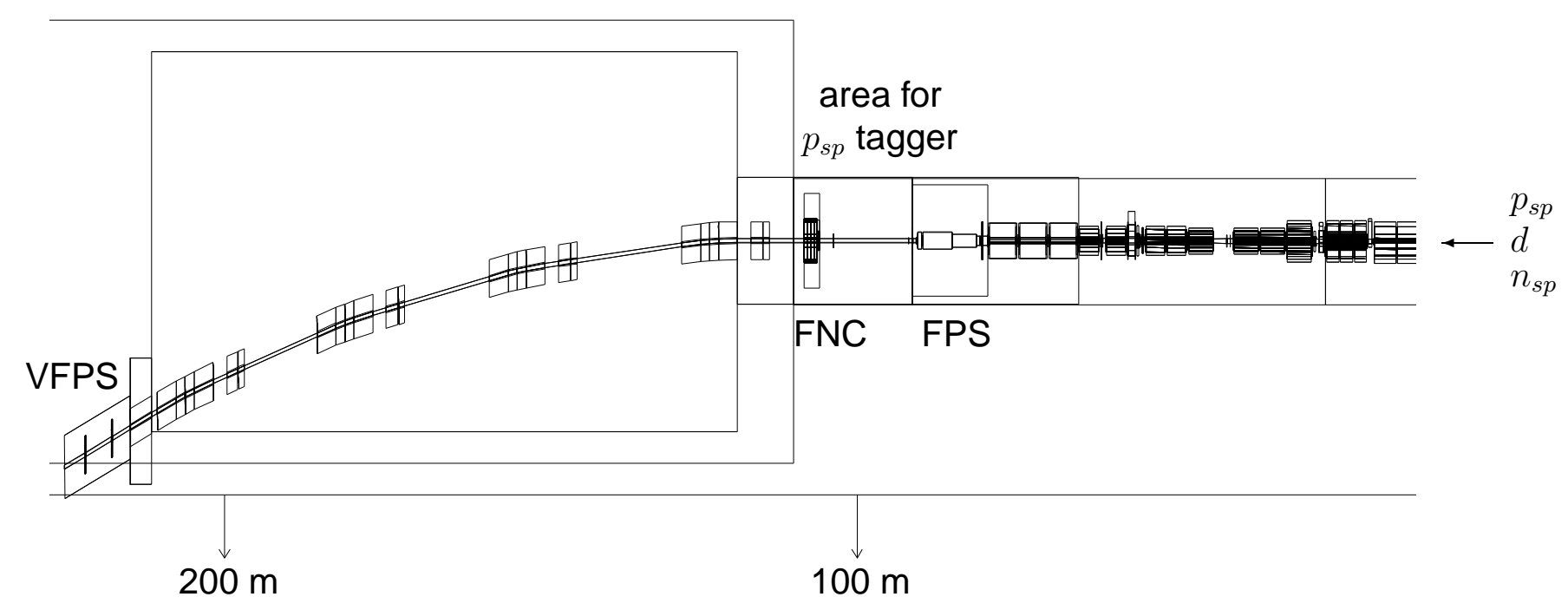

Figure 31: Beamline geometry in the upstream of the $\mathrm{H} 1$ detector in the proton direction.

HERA programme, only the upgrade to allow proton spectator tagging would be made. If future running were to be started after a longer shutdown, perhaps to prepare new injection lines for HERA, the removal of the GO and GG magnets and could be considered and all the upgrades mentioned here could be performed.

\subsection{Detecting spectator nucleons, an upgrade of the $\mathrm{H} 1$ forward tagging system}

The ability to identify leading forward particles is an essential requirement for the deuteron programme at HERA [150]. For example, the deep inelastic scattering of an electron off the proton in the deuteron will result in a forward going "spectator" neutron carrying approximately half the deuteron beam momentum, whereas scattering off the neutron would produce a spectator proton. Detection of these particles thus allows the identification of the scattering process. The requirement that diffractive interactions also be identified complicates matters somewhat. The diffractive interaction could be coherent, in which case the deuteron remains intact and loses only a small fraction of its momentum, $x_{\mathbb{P}}$, i.e. it proceeds down the beampipe with a fraction $z=1-x_{\mathbb{P}}$ of the beam momentum. Alternatively, the diffractive interaction could involve only the proton, it is then the proton which loses a proportion $x_{\mathbb{P}}$ of its momentum and both the proton and the neutron will then continue down the beam pipe. The final possibility is that the diffraction occurs off the neutron which loses a fraction $x_{\mathbb{P}}$ of its momentum and again, the proton and neutron continue down the beam pipe. Three distinct sources of leading particles 
must thus be identified and measured. These are characterised by the fraction of the deuteron beam momentum carried by the leading particle, as follows:

- Diffractively scattered deuterons arising from the coherent diffractive process $e d \rightarrow e d X$, with $z=1-x_{\mathbb{P}}$.

- Spectator protons $p_{s p}$ and neutrons $n_{s p}$, arising during the interactions $e n \rightarrow e X$ and $e p \rightarrow e X$, respectively. The spectator nucleons have $z \approx 0.50 \pm 0.03$ with the spread arising from the Fermi motion of the nucleons bound within the deuteron of typically $30 \mathrm{GeV}$ (see figure 10, left.)

- Diffractively scattered protons and neutrons, arising during the processes $e p \rightarrow e X p$ and $e n \rightarrow e X n$, respectively. These have $z \approx 0.50\left(1-x_{\mathbb{P}}\right) \pm 0.03$, where $x_{\mathbb{P}}$ this time refers to the fractional energy loss in the diffractive $e N$ scattering and the smearing again arises from the Fermi motion.

As is illustrated in figure $31, \mathrm{H} 1$ already has in place detectors that are adequate for the detection of particles from some of these sources. For the first case, diffractively scattered deuterons, the Very Forward Proton Spectrometer (VFPS) detector is fully adequate (though it may have to be renamed). The VFPS has almost $100 \%$ acceptance in a well defined range of $x_{\mathbb{P}}$ and, since it covers the low $t$ region, it is well matched to the very steeply falling $t$ dependence expected for diffractively scattered deuterons (exponential $t$ slope $b_{d} \propto R_{d}^{2}$, the squared deuteron radius $\left.\approx 4 b_{p}\right)$. On the other hand, the VFPS $t$-resolution of $\mathcal{O}\left(0.2 \mathrm{GeV}^{2}\right)$ will be insufficient to measure the $t$-dependence of the reaction $e d \rightarrow e d X$.

Detecting final state nucleons produced as spectators, or through diffractive $e N$ scattering, requires taggers sensitive in the region around $z=0.5$. The present Forward Neutron Calorimeter (FNC) [151] can be used as a neutron detector without further modification. It has an intrinsic $p_{t}$ resolution of $\sigma\left(p_{t}\right) \simeq 12 \mathrm{MeV}$ for neutrons observed in the pre-shower detector (probability $\sim 80 \%$ ) and $\sigma\left(p_{t}\right) \simeq 45 \mathrm{MeV}$ in the case that all the energy is deposited in the main calorimeter (probability $\sim 20 \%$ ). However, the effects of the beam angular divergence at the interaction point must also be considered. For the present HERA II optics, including the new focusing magnets, the divergence is $\sigma\left(\theta_{x}\right)=0.046 \mathrm{mrad}$ and $\sigma\left(\theta_{y}\right)=0.168 \mathrm{mrad}$ [152], leading to uncertainties in $p_{x}$ and $p_{y}$ of the order of $20 \mathrm{MeV}$ and $75 \mathrm{MeV}$ respectively. The geometrical acceptance of the FNC is limited by the aperture of the $B U_{L}$ magnets between $z=66$ and $79 \mathrm{~m}$ (figure 31). The best acceptance is achieved when running with non-zero deuteron beam tilts of $\theta_{x}=-0.2 \mathrm{mrad}$ and $\theta_{y}=0.2 \mathrm{mrad}$. Figure 32 shows the simulated acceptance as a function of the leading neutron's energy and transverse momentum with and without this beam tilt. In the optimal case, acceptances in excess of $90 \%$ can be achieved for $z \sim 0.5$ and $p_{t}<0.1<\mathrm{GeV}$.

\subsection{The proton spectator tagger}

The detector that must be added to the $\mathrm{H} 1$ apparatus is a proton tagger sensitive in the region $z \sim 0.5$, the Proton Spectator Tagger (PST). As shown in figure 33, as far as the beam optics are concerned, this device could exploit the dispersion in the horizontal plane in the region around $z=70 \mathrm{~m}$ or in the vertical plane around $z=90 \mathrm{~m}$. In both cases, the sensitive region lies 

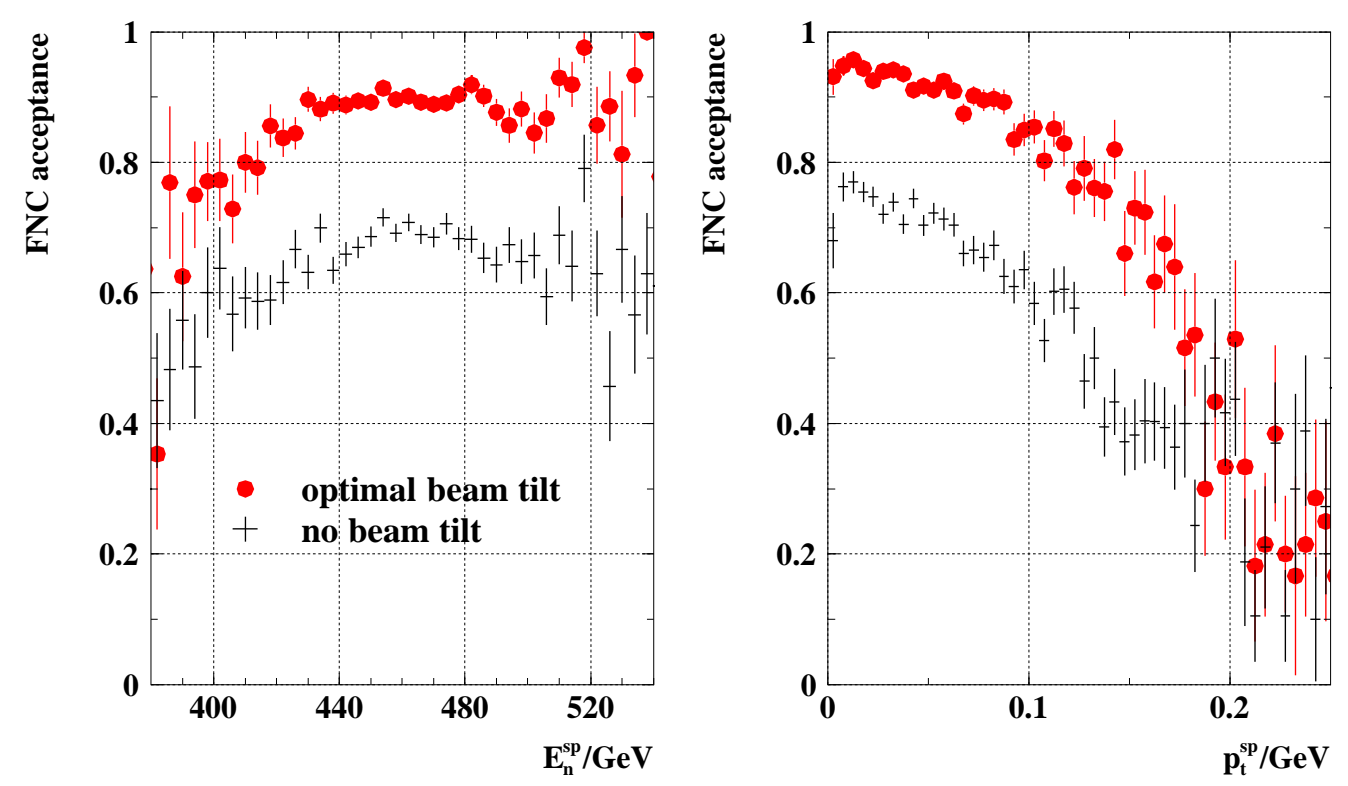

Figure 32: FNC acceptance for final state neutrons as a function of their energy and transverse momentum (for current HERA II $e^{+}$optics).

outside the beampipe. Considering the location of the major beampipe components (figure 31), the most promising location seems to be at around $z=90 \mathrm{~m}$.

A possible design for this detector has been investigated. This is composed of two stations, one at $z=80 \ldots 85 \mathrm{~m}$ and a second at $z=95 \ldots 105 \mathrm{~m}$. Each station consists of two subdetectors, designed to reconstruct track segments in the $x$ and $y$ coordinates at their respective locations. The transverse size of the station at $80 \ldots 85 \mathrm{~m}$ is $13 \times 6.5 \mathrm{~cm}^{2}$ in $x$ and $y$, respectively, that at $95 \ldots 105 \mathrm{~m} 13 \times 13 \mathrm{~cm}^{2}$, in accordance with the expected distribution of spectator proton positions at these locations, as illustrated in figure 34. The coordinate planes of $13 \mathrm{~cm}$ width each consist of 64 fibres of $2 \mathrm{~mm}$ diameter and of square section, read out through a 64-channel position sensitive photo-multiplier, as used in the current H1 FPS. This gives a spatial resolution of 0.3 to $0.4 \mathrm{~mm}$. The $6.5 \mathrm{~cm}$ wide coordinate plane is constructed from 4 layers, each of which contains 64 scintillating fibres of $1 \mathrm{~mm}$ diameter and square section, with successive planes being offset by 1 fibre radius. Four fibres, one from each layer and adjacent in $z$, are readout through one pixel of a 64 channel position sensitive photo-multiplier, as is shown in figure 35 . The resulting spatial resolution is 0.15 to $0.2 \mathrm{~mm}$. A $1 \%$ change of the proton energy at the interaction point results in a deviation in $x$ and $y$ at the $85 \mathrm{~m}$ station of 0.2 to $0.25 \mathrm{~mm}$ and of 0.5 to $0.5 \mathrm{~mm}$ at $95 \mathrm{~m}$, so the proposed detector allows a measurement of the spectator proton's momentum with a precision of better than $1 \%$. If the track segments in each station are required to consist of 3 hit layers out of a possible 4 , the reconstruction efficiency for each coordinate is in excess of $98 \%$ and the track reconstruction efficiency including linking between the two stations is better than $90 \%$. The rate of spurious tracks which match this requirement is negligible.

Calibration of the system can be performed on a fill-by-fill basis using elastic $\rho$ meson production off the proton in the deuteron. Measurements of $\rho$ mesons in the H1 central detector 

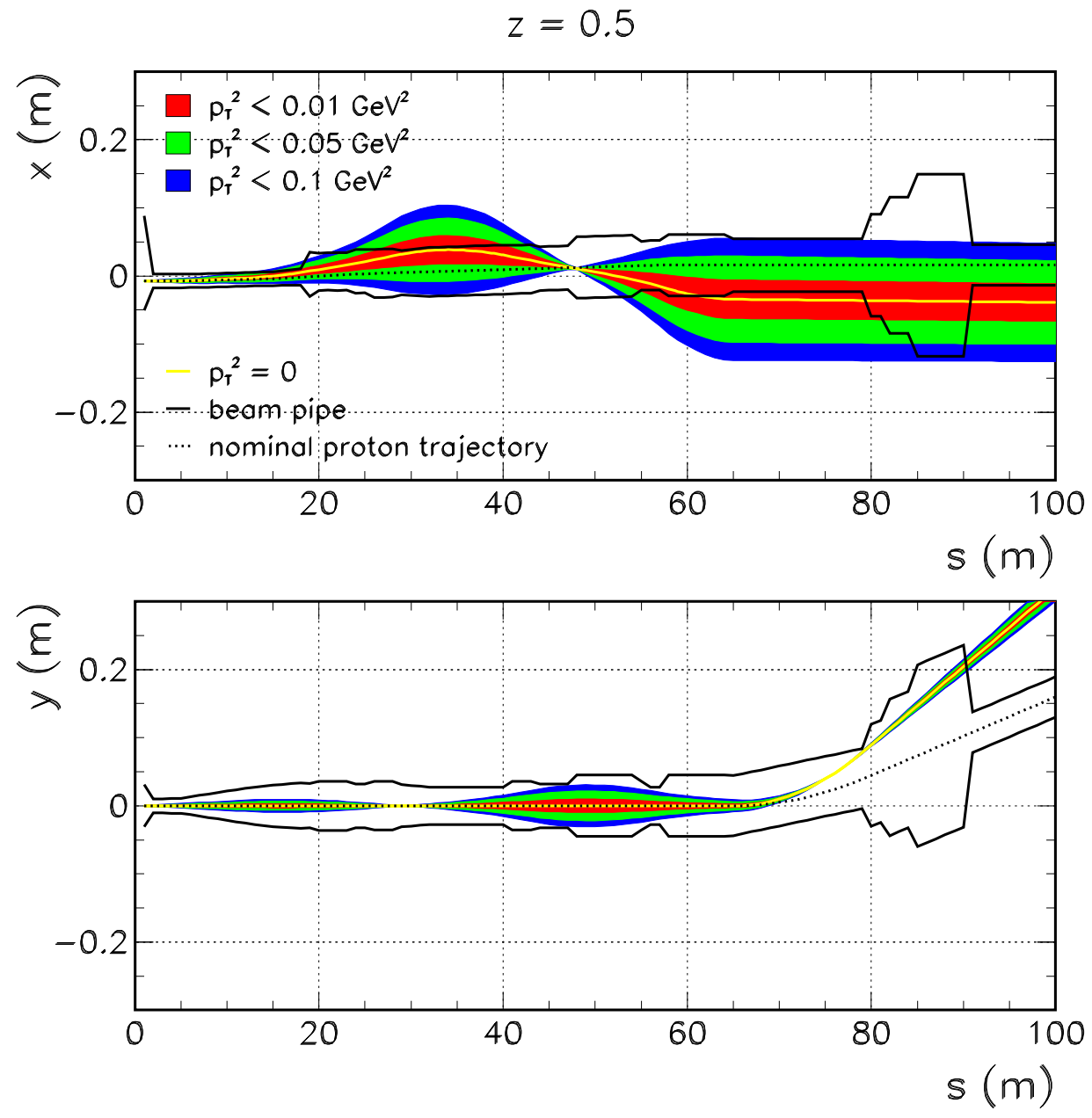

Figure 33: Trajectories of protons in the horizontal and vertical planes for the current HERA II optics. 

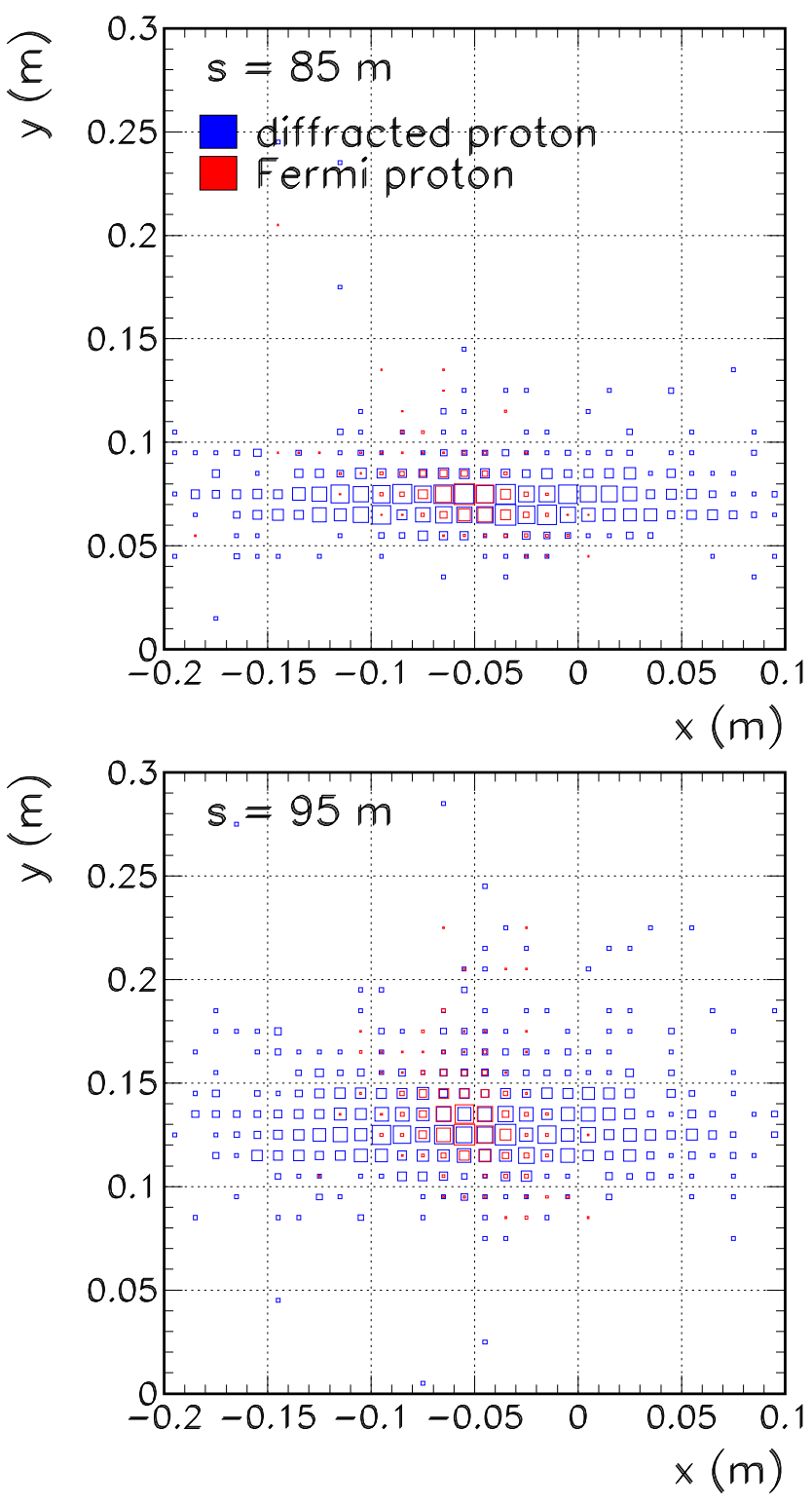

Figure 34: Distribution in $x$ and $y$ of protons at $z=85$ and $95 \mathrm{~m}$ which have undergone diffractive interactions ("diffracted protons") or whose neutron partners in the deuteron have suffered an inelastic scatter ("Fermi protons") at the interaction point. 


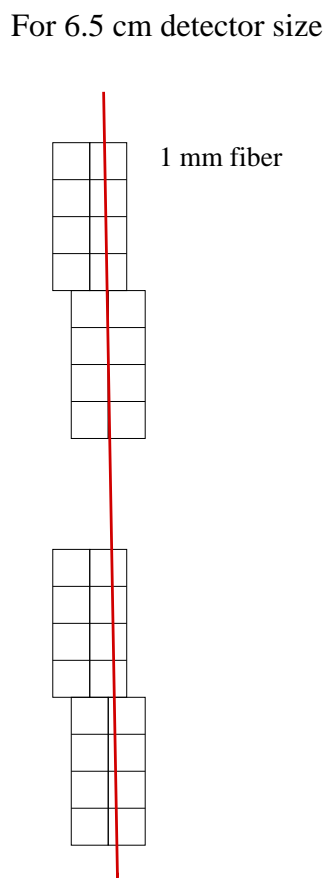

For $13 \mathrm{~cm}$ detector size

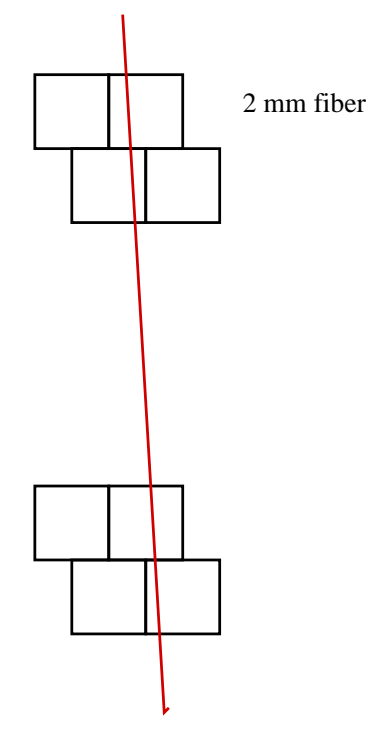

Figure 35: Schematic view of the scintillating fibre detectors of the Proton Spectator Tagger.

in coincidence with the scattered proton in the PST provide a set of constraints which can be used to determine the transverse position of the scintillator planes with respect to the deuteron beam. Trigger signals can be provided by the PST using scintillating tiles placed in front of and behind each sub-detector, read out using photo-multipliers without multiplexing, as is currently done for the horizontal FPS station.

The acceptance of the PST, if used with the HERA II optics without modification of the beam pipe is below $10 \%$. This can be improved to $45 \%$ if minor modifications to the beam pipe are made. Further improvements can be made by optimising the alignment of the proton beam, in particular its position within the BU magnets downstream of $\mathrm{H} 1$. The maximum achievable value of the acceptance is then $95 \%$, with the value at zero beam tilt being $70 \%$. Further improvements may be obtained if the super conducting magnets GO and GG are removed from the $\mathrm{H} 1$ interaction region, but these have yet to be studied in detail.

The total costs associated with the construction of the PST amount to 100 kEuro. A breakdown of these costs is given in table 2 .

Even with full acceptance taggers, spectator nucleons and diffractively scattered nucleons cannot be separated on an event-by-event basis and some input from statistical methods will be required. To illustrate the ambiguities, the observation of an intact neutron in the final state scattered through a small angle and having $z \sim 0.5$ could imply any of:

1. An inelastic scattering from the proton with the neutron as spectator.

2. An elastic scattering from the proton with the neutron as spectator. 


\begin{tabular}{|l|c|}
\hline Item & Cost (kEuro) \\
\hline $\begin{array}{l}\text { Sixteen 64-channel position sensitive photo-multipliers } \\
\text { (R5900-M64 Hamamatsu) plus 16 trigger photo-multipliers }\end{array}$ & 35 \\
(16 R5600 Hamamatsu) & 35 \\
Fibre hodoscopes & 20 \\
Mechanics, platforms & 10 \\
Upgrade of electronics, CAEN HV modules, cables & 100 \\
\hline Total & \\
\hline
\end{tabular}

Table 2: Costs for the construction of the Proton Spectator Tagger.

3. An elastic scattering from the neutron with the proton as spectator.

It will usually be possible to distinguish possibility 1 from possibilities 2 and 3 on the basis of the presence or absence of a large rapidity gap close to the outgoing beam direction using similar selection methods to those currently employed for diffractive measurements [153]. Possibilities 2 and 3, where both nucleons are present in the final state, can be distinguished with rather high efficiency by using the $z$ and $p_{t}$ measurements of the scattered protons and neutrons. Since $x_{\mathbb{P}}$ for a diffractive process is typically of the same size as the smearing due to the Fermi motion and since the energy resolution of the FNC is insufficient, measurements of the $z$ variable are not likely to be particularly helpful. Distinguishing on the basis of the very different distributions in $p_{t}$ of the observed nucleons is much more promising. As illustrated in figure 32 (right), the $p_{t}$ distribution of spectator nucleons is confined to very low values. Figure 36 shows the $p_{t}^{2}$ distribution of spectator neutrons observed in the FNC from a simulation of deuteron scattering events. Even after including the effects of the beam divergence at the interaction point and the $p_{t}$ resolution of the FNC, a rather good separation seems possible with appropriate cuts. A similar separation is possible with PST stations sensitive near $z=0.5$.

Determining the precision possible for comparisons of $e p$ and $e n$ scattering will require a detailed feasibility study, which has yet to be performed.

\subsection{Upgrade to the forward region in $\mathrm{H1}$}

\subsubsection{Introduction}

This proposal to upgrade the $\mathrm{H} 1$ detector requires that the super-conducting quadrupole magnets GG and GO be removed, i.e. that HERA I optics be used. The goal is to cover the angular range from the edge of the LAr calorimeter at $4^{\circ}$ down to $0.3^{\circ}$. To extend the detector coverage down to such small angles, a system of several calorimeter-trackers is proposed which will be placed 


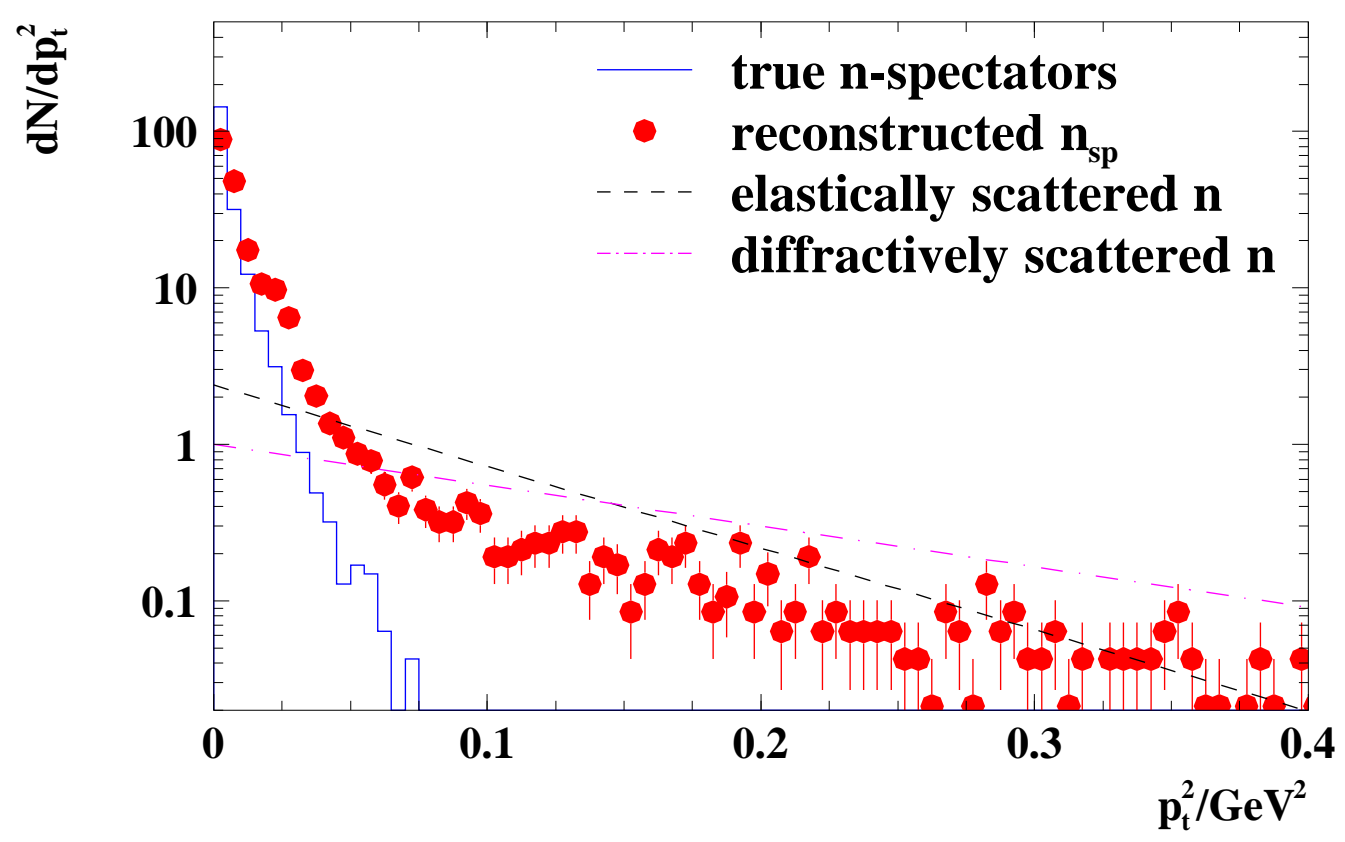

Figure 36: Simulation of the $p_{t}$ distribution of spectator neutrons observed in the FNC including the effects of the current HERA II beam divergence and the FNC resolution. The expected contributions from spectator and diffractively scattered neutrons, without experimental uncertainties, are indicated separately. 


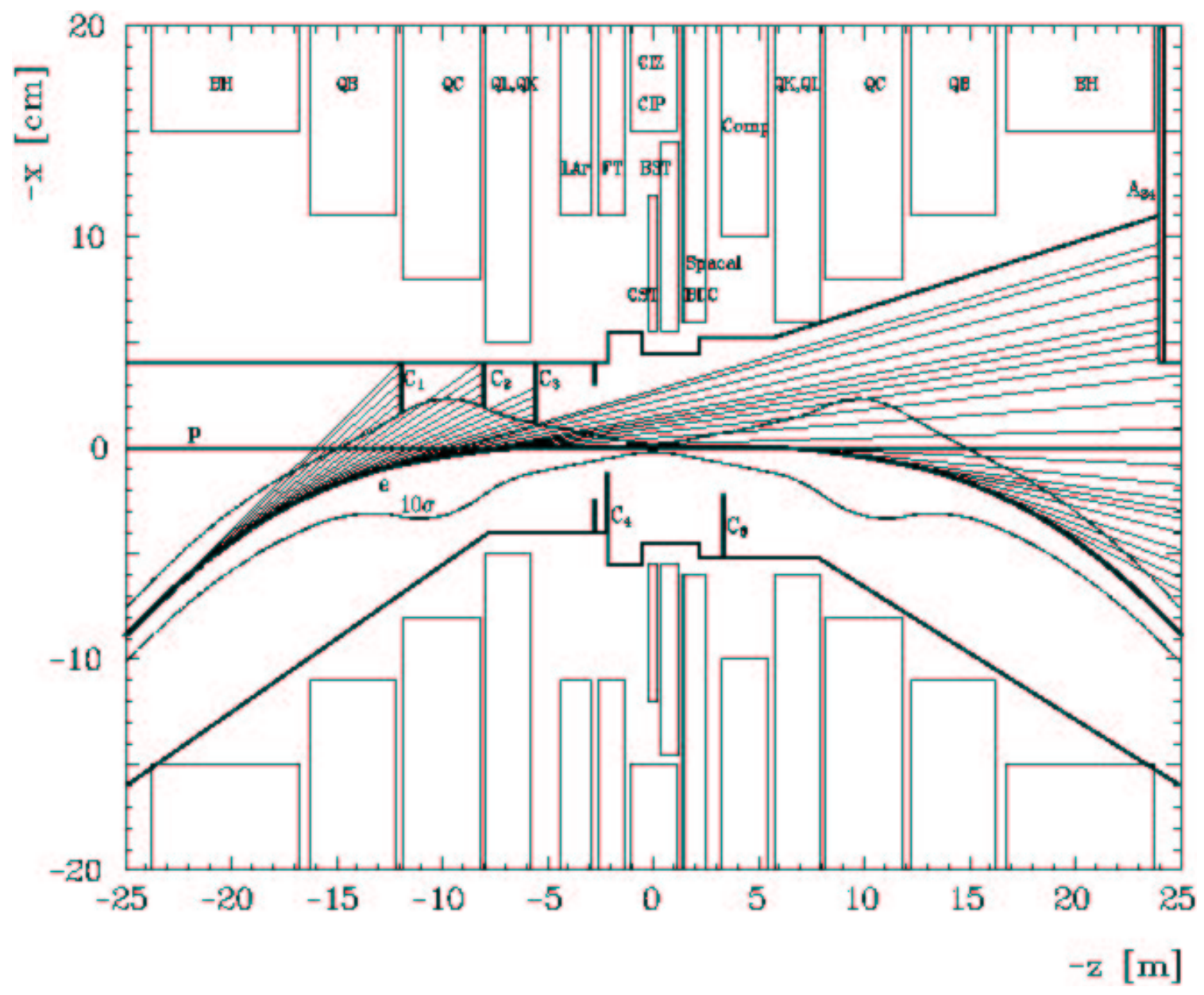

Figure 37: Schematic view of the layout of HERA for HERA I optics, showing a top view of the $\mathrm{H} 1$ interaction region. 
in the space made available by the removal of the superconducting magnets inside the forward track detector (FTD). The general layout is shown in figure 38 and the geometrical specifications of the various detectors are given in table 3 . In order to get combined information on particle trajectories and energy, we are considering the use of digital calorimeters (FTC1 and FTC2). The amount of dead material in front of the digital calorimeters, given by flanges, the LAr cryostat etc. can be corrected for by mounting preshower detectors (FPC1 and FPC2) directly outside the vacuum pipe. We also propose to determine the trajectories of the particles entering the preshower detectors by instrumenting the beam pipe, possibly by the insertion of a system of circular fibre counters at various positions along the beam line. This challenging detector concept requires detailed investigation, but given the physics advantages of full calorimetric coverage down to very small angles, such concepts deserve further investigation. In figure 38 this scenario is indicated, but it will be not be discussed further here.

\begin{tabular}{|l|c|c|c|}
\hline Calorimeter & $z$-position $(\mathrm{m})$ & radius $(\mathrm{cm})$ & polar angle \\
\hline FPC1 & $1.25-4.5$ & $4.5-9.5$ & $0.7-2$ \\
\hline FTC2 & $4.75-5.5$ & $4.5-25$ & $0.7-3$ \\
\hline FPC2 & $8.3-10.3$ & $4.5-11$ & $0.3-0.7$ \\
\hline FTC2 & $11.0-11.7$ & $4.5-16$ & $0.3-0.7$ \\
\hline
\end{tabular}

Table 3: The $z$ positions, radial dimensions and the angular coverage of the various proposed calorimeters.

\subsubsection{Preshower detectors}

The preshower detectors (FPC1 and FPC2) are designed to enable a correction to be made for the energy lost in the flanges, the LAr cryostat, the beam pipe and other dead material in front of the digital calorimeters (FTC1 and FTC2). The FPC1 detector will be located in the space between the beam pipe and the inner wall of the LAr cryostat and the forward tracker FTD. The size is defined by the available space, giving a radial extension from $3.2 \mathrm{~cm}$ to $8.5 \mathrm{~cm}$ and covering the region from $z=130$ to $450 \mathrm{~cm}$. A possible solution for the proposed detector would be a sandwich type electromagnetic calorimeter with fine segmentation in the $\phi$ direction and relatively coarse longitudinal segmentation. A schematic view is shown in figure 39 . The detector consists of 5 sections in the $z$ direction, each section consisting of $24 \phi$ towers. A tower consists of 6 alternating layers of scintillator tiles of thickness $1.2 \mathrm{~mm}$ and lead of thickness $\sim 10 \mathrm{~mm}$. The widths of the scintillator tiles vary between $10 \mathrm{~mm}$ and $20 \mathrm{~mm}$, depending on their radial position. The lead absorbers have the shape of half-cylinders. The light from each scintillator tile is collected by a $1.2 \mathrm{~mm}$ thick wavelength shifter fibre (WLS), positioned in a groove along one side of the tile, and is guided by clear fibres to the photodetectors. All 6 tiles from one tower are read out by one photodetector. In total the calorimeter will have 120 readout channels. Since the photo-detectors need to function in a magnetic field of $1.2 \mathrm{~T}$, the use of silicon photomultipliers or multichannel Hamamatsu photomultipliers could be considered. The readout could be done through the present data SpaCal data acquisition system.

The energy resolution of such a setup (FPC1 and FTC1) will be of the order of $100 \% / \sqrt{E}$. This number comes from an analysis of data from the H1 Forward Neutron Calorimeter (FNC), 


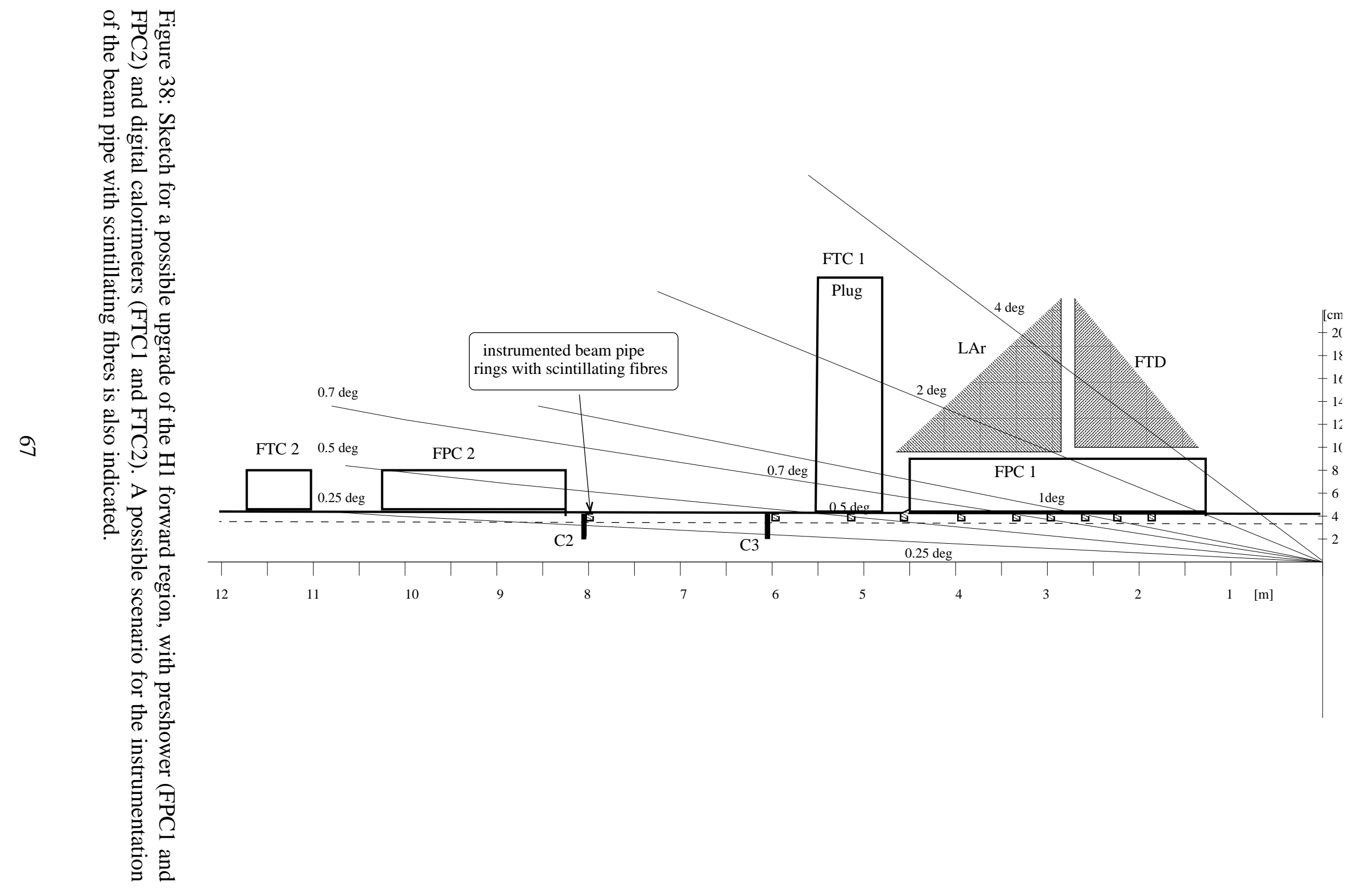




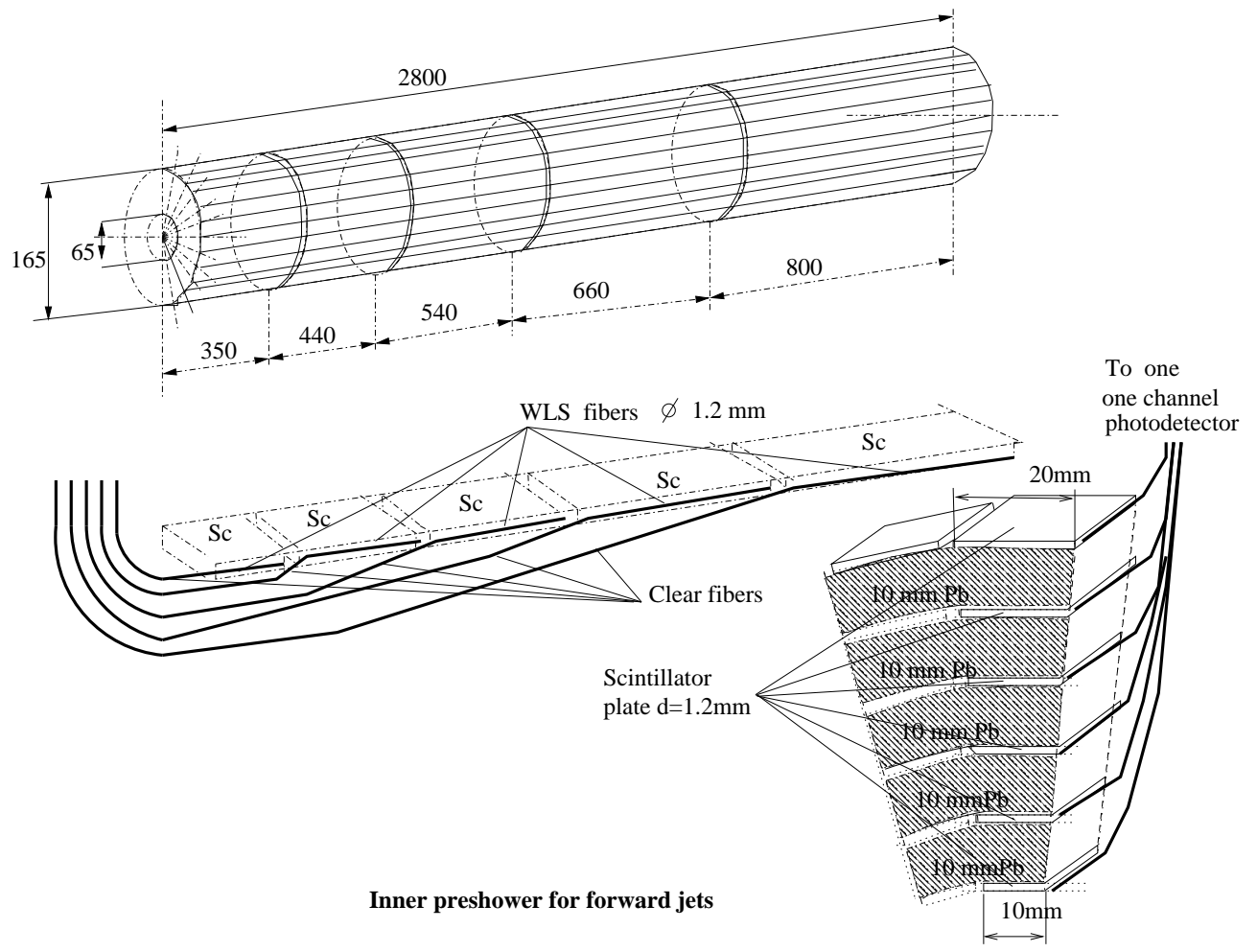

Figure 39: Sketch of a possible forward preshower calorimeter

which is a lead-scintillator sandwich calorimeter consisting of towers with transverse sizes $20 \times$ $20 \mathrm{~cm}^{2}$ in 5 longitudinal sections $(1 \times 1.5 \lambda$ and $4 \times 2 \lambda)$. Thus, the basic structure of the FNC detector is similar to that of the proposed setup. In order to model the effects of the flanges etc. between FPC1 and FTC1, one of the FNC sections was treated as dead material

A similar detector with different longitudinal segmentation can be used in the region between $8 \mathrm{~m}$ and $11 \mathrm{~m}$ to cover polar angles between $0.25^{\circ}$ and $0.7^{\circ}$.

\subsubsection{Digital calorimeter}

The extreme forward direction in deep inelastic scattering events is characterised by a high density of very energetic particles, the detection and measurement of which is particularly challenging. The combined tracking and calorimetric capabilities of digital calorimeters may offer some advantages in this region.

It has been recognised that, if the cells in a calorimeter are small enough, a good estimate of the energy is obtained by just counting the number cells in which energy has been deposited. Tests with calorimeters based on the digital technique have given resolutions similar to those of sampling calorimeters with analogue readout. $\mathrm{H} 1$ has demonstrated that this technique works using the LAr calorimeter, as is shown in figure 40. Here, in the most finely segmented inner forward (IF) section of the LAr, the number of cells which have contributed to an energy measurement is plotted against the measured energy. A very good correlation is observed. Due to 


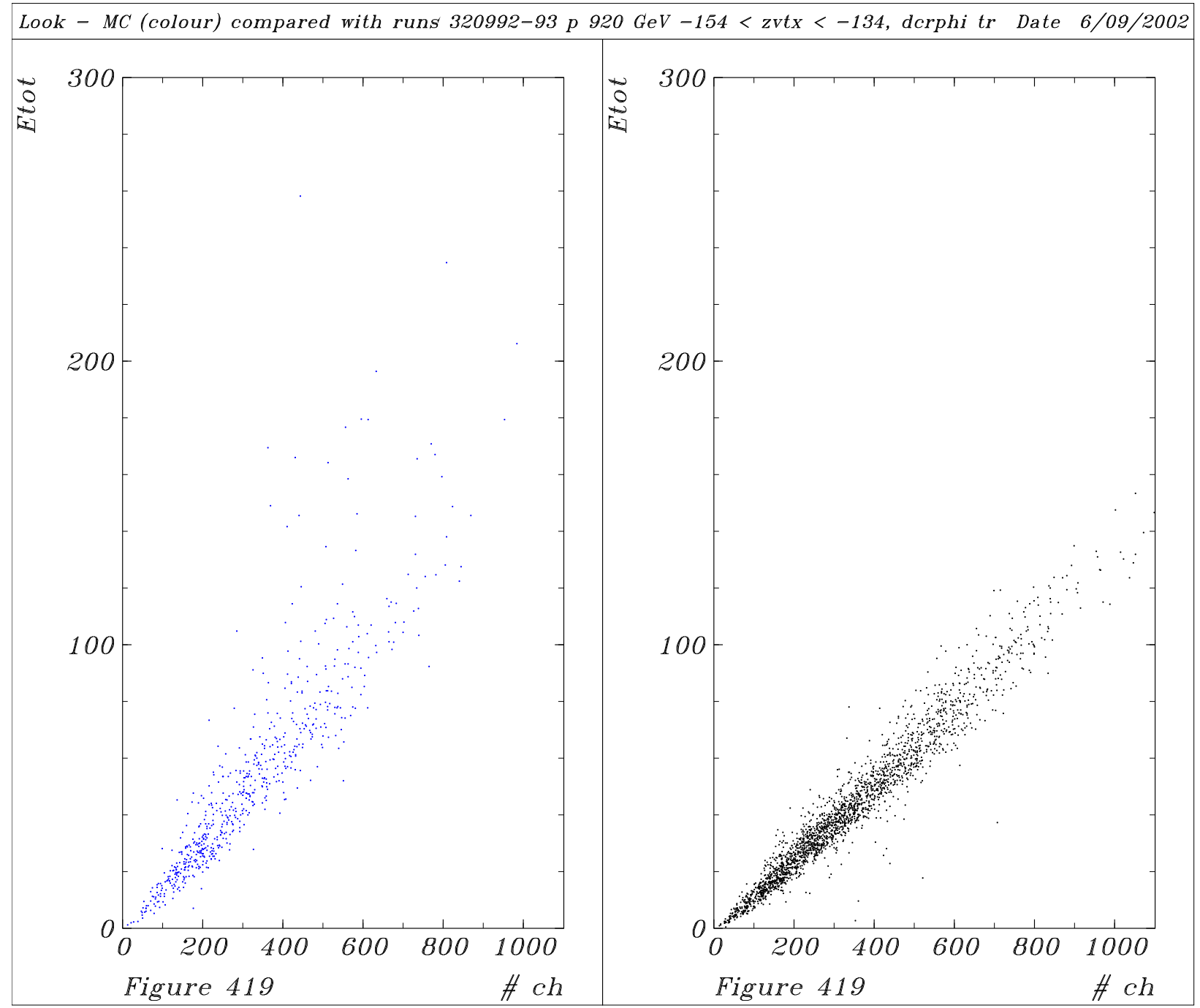

Figure 40: Correlation of the number of cells with the energy in the H1 LAr calorimeter: left Monte Carlo; right data. 
the highly segmented readout, information on the particle direction is also obtained. This aids the application of dead material corrections.

First studies propose that a sandwich hadron calorimeter is used, with absorber plates made of stainless steel and interleaved detector layers. These could be resistive plate chambers or wire chambers operated in the limited Geiger or proportional modes. The signals are collected on small pads. In the first two cases, the signal is big enough to switch shift registers without amplification, in the second additional electronic amplification is needed. The bits are read out serially. The response of such a detector has been discussed and simulated in detail in the context of the TESLA detector. It was shown that the purely digital signal is linear in energy and that the resolution can be as good as that achieved using analogue readout if, in addition to the information on the number of cells that have fired, the density of cells, the correlations between them etc. are used.

\subsection{Upgrade to the backward region}

Measurements at low $x$ and $Q^{2} \lesssim 5 \mathrm{GeV}^{2}$, cannot be performed with the $\mathrm{H} 1$ detector in its current configuration. The superconducting GG magnet, necessary to provide the focussing required to get high luminosity for HERA II running, sits within the backward spaghetti calorimeter (SpaCal) and restricts the experiment's acceptance in this region, into which electrons are scattered at low $Q^{2}$ and $x$. Hence, removal of this magnet and the use of the resulting space for an upgrade of the backward region are necessary for the future low $Q^{2}$ and $x$ physics programme. This upgrade has to combine precision tracking with high resolution calorimetry. Here, we describe a design which is based on the technologies already in use within H1.

\subsubsection{Physics requirements}

The experimental aim of the new phase of low $x$ measurements is to achieve high precision and to extend the acceptance down to squared four-momentum transfers as small as $Q^{2} \simeq 0.1 \mathrm{GeV}^{2}$. Larger $Q^{2}$ values can be measured with the existing SpaCal and tracking devices. The main requirements can be summarised as follows:

- Measurement of the energy of the scattered electron to within $0.2 \%$ to $1 \%$ in the range $E_{e}>E_{e}^{\prime} \gtrsim 2 \mathrm{GeV}$. This can be satisfied with a very backward electromagnetic spaghetti calorimeter (VBES).

- Measurement of the polar angle of backward scattered tracks down to scattering angles of $0.2 \mathrm{mrad}$. This requires a silicon tracker. For alignment purposes, a further tracking device attached to the VBES is also essential, here this is assumed to be a proportional chamber similar to the current backward proportional chamber (BPC).

- Control of efficiencies to within $0.5 \%$. This requires redundancy in the tracking and the SpaCal calibration procedure. 
- Measurement of the charge symmetry of the low energy photoproduction background to $1 \%$ accuracy. This requires high statistics and high resolution (silicon) tracking preceding the calorimeter. Keeping the electron tagging (luminosity) system to allow independent measurement of the photoproduction background is also desirable, even though a significant part of this will be measured in the new backward spectrometer itself.

- Measurement of the luminosity to better than $1 \%$ precision. Bethe-Heitler scattering will be measured by the new backward spectrometer and by the current luminosity system. This redundancy will improve the luminosity measurement accuracy beyond the currently achieved level of $1.5 \%$. The $\mathcal{O} \alpha^{3}$ corrections to the cross section measurement will have to be calculated and used in the luminosity determination as the lack of these currently causes a $0.5 \%$ theoretical uncertainty in the HERA luminosity measurement.

- Reconstruction of the energy-momentum balance of the event by measuring the variable, $y_{h}=\left(E-p_{z}\right) / 2 E_{e}$. This has been achieved in the $\mathrm{H} 1$ detector by combining tracking with accurate LAr calorimetry, with a precision of $1 \%$ for the electromagnetic and $2 \%$ for the hadronic energy calibration. This reduces the radiative corrections that must be made to inclusive cross section measurements to a tolerable level and allows the redundant reconstruction of the kinematics.

The resulting measurement uncertainty for the inclusive cross section is estimated to be below $1 \%$ in the medium $y$ range and to reach a maximum of $2 \%$ at the highest $y$ values. In the following, the main items of the upgraded $\mathrm{H} 1$ backward apparatus are briefly presented.

\subsubsection{Modifications to the H1 backward region}

A sketch of the backward spectrometer is shown in figure 41. It uses the space freed up by the removal of the GG magnet and also that that was taken up by the compensator coil during HERA I running. The HERA machine group inform us that this will not required, even if we return to HERA I optics. Accurate measurements in the "transition region", $Q^{2} \sim 1 \mathrm{GeV}^{2}$, require that the acceptance be smooth. The proposal is thus to move a sizeable part of the SpaCal calorimeter [154] backwards so that is has full acceptance for polar angles between $173^{\circ}$ and $179^{\circ}$, for an inner beam pipe radius of $3 \ldots 4 \mathrm{~cm}$. The kinematic coverage of this device is illustrated in figure 42.

\subsubsection{The very backward spectrometer}

The general layout, as shown in figure 41, comprises three systems:

1. The very backward silicon tracker (VBST) together with the very very backward silicon tracker (VVBST).

2. The very backward electromagnetic SpaCal (VBES).

3. The very backward proportional chamber (VBPC). 


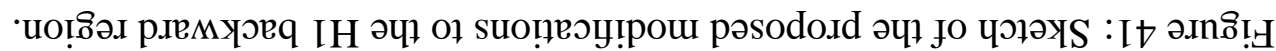

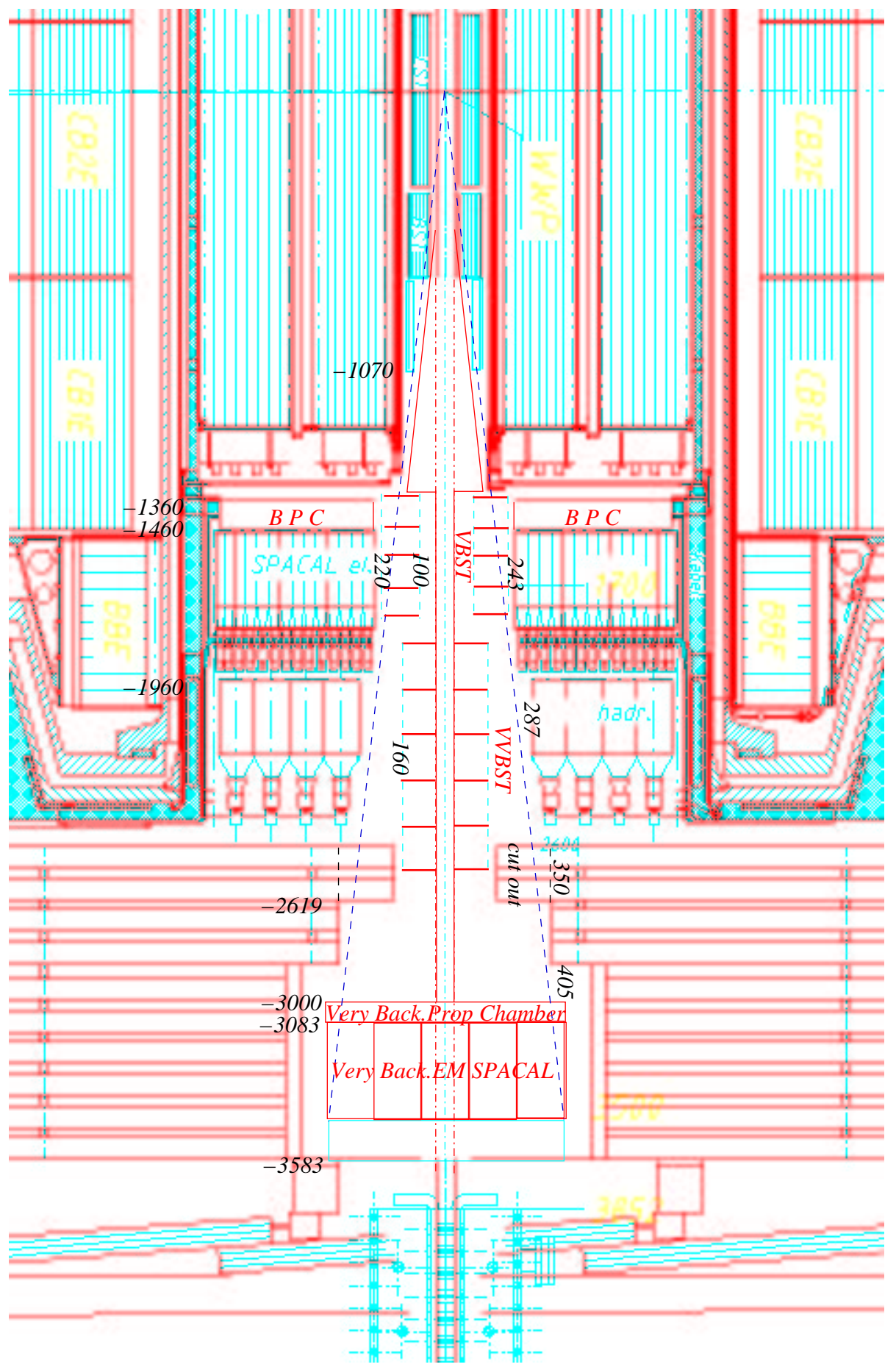



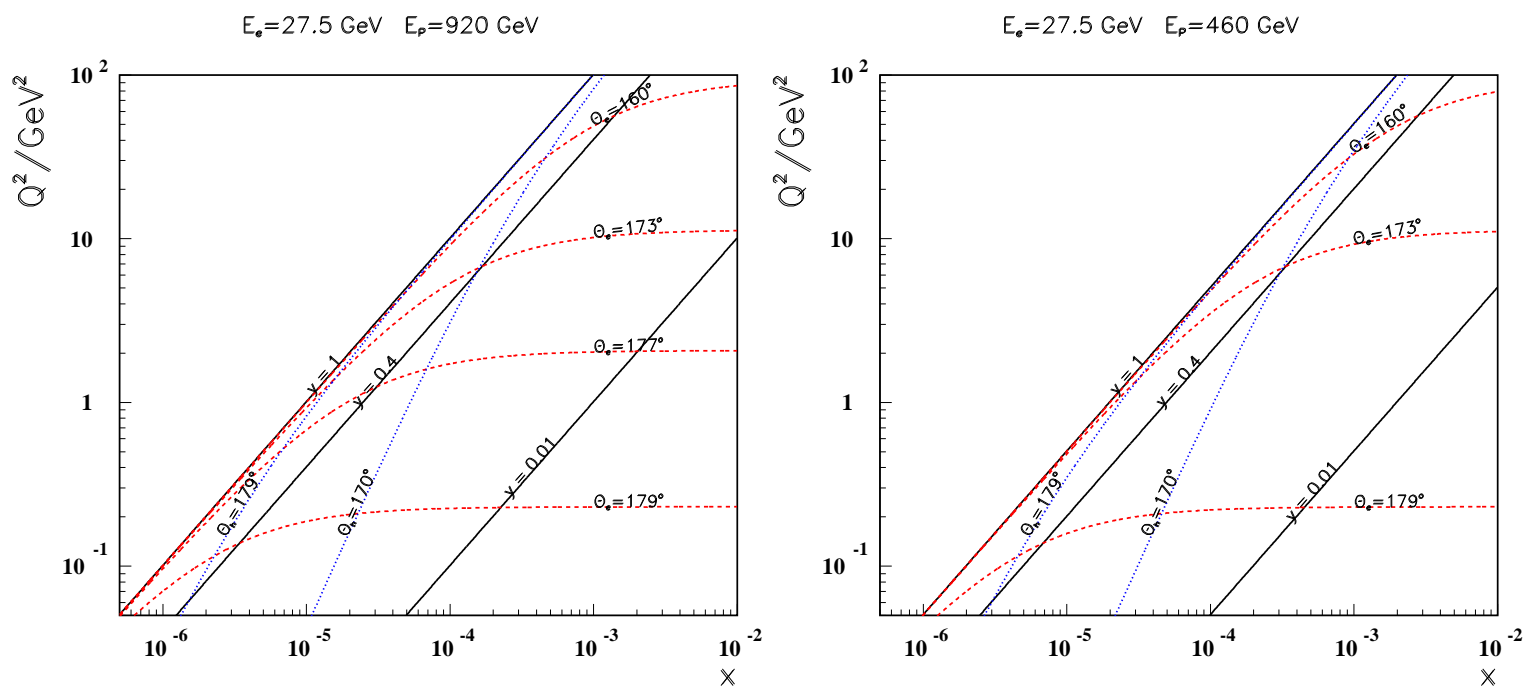

Figure 42: Kinematic coverage for ep scattering of the $\mathrm{H} 1$ apparatus extended in the backward direction for the default beam energy (left) and for a proton beam energy reduced by a factor of two (right). The very backward electromagnetic SpaCal (VBES) and associated tracking detectors have an acceptance between about $173^{\circ}$ and $179^{\circ}$ which covers the transition region from deep inelastic scattering to photoproduction, i.e. from about $Q^{2}=10 \mathrm{GeV}^{2}$ down to $Q^{2}=0.2 \mathrm{GeV}^{2}$ at large $x$ and shifted towards lower $Q^{2}$ values at small $x$. At large values of the inelasticity, $y$, which correspond to small energies $E_{e}^{\prime}$ of the scattered electron $\left(y \simeq 1-E_{e} / E^{\prime}\right)$, the hadronic final state at low $Q^{2}$ is also scattered into the backward direction. 


\begin{tabular}{|l|c|}
\hline VBPC front face position & $-300.0 \mathrm{~cm}$ \\
\hline VBES front face position & $-308.3 \mathrm{~cm}$ \\
\hline VBES radius & $40.5 \mathrm{~cm}$ \\
\hline Beam pipe radius in VBPC/VBES region & $4.0 \mathrm{~cm}$ \\
\hline
\end{tabular}

Table 4: Parameters of the new very backward calorimeter and very backward proportional chamber.

SpaCal technology is an obvious choice for the very backward calorimeter due to its high resolution, stability and triggering capability. Moreover, use of this technology ensures an homogeneous and straightforward approach to data handling (readout, trigger and calibration), simulation and reconstruction, achieved by incorporating the VBES into the existing SpaCal environment.

The required angular coverage is obtained by removing $3 \times 3$ inner supermodules of the electromagnetic SpaCal. These can be used for the VBES. In addition, the 16 innermost cells of the hadronic SpaCal are removed and the opening in the backward iron shells is widened at the position $z=-261.9 \mathrm{~cm}$ to $35 \mathrm{~cm} \times 35 \mathrm{~cm}^{10}$. In the arrangement sketched in figure 41 , the small acceptance gap between the main electromagnetic SpaCal and the VBES will be covered by exploiting the variation in $z$ of the position of the primary event vertex.

The dimensions of the proposed configuration are given in table 4.

\subsubsection{Very backward silicon tracking}

Tracks emitted in the backward direction can be reconstructed using silicon strip detectors, as has been successfully done with the BST at H1 [155]. The high resolution and redundancy provided by 4 to 5 spatial hits, together with the measurements from the planar proportional chamber, allow the reconstruction of the $z$ vertex and hence the polar angle of the scattered electron with high accuracy using only the electron track. This is necessary in order to reduce the background from photoproduction events in which a $\pi^{0} \rightarrow \gamma \gamma$ decay fakes the signal of a scattered electron at high $y$, and in order to reduce the dependence of the vertex reconstruction on central tracks at low $y$, which has previously been a major limitation in cross section measurements at large $x$. A rather uniform acceptance can be achieved with two modules, one (the VBST) covering the angular range from about $173^{\circ}$ to $177^{\circ}$, and the other (the VVBST) covering the lowest $Q^{2}$ region corresponding to angles of about $177^{\circ}$ to $179^{\circ}$. The sensors can be made of single sided $6^{\prime \prime}$ wafers, mounted back to back, which ensures high signal to noise performance and homogenous acceptance. The main parameters of this design are summarised in table 5 .

Near the beam axis, the $\mathrm{H} 1$ solenoidal field is still rather uniform even in the very backward region, its value reduces from $1.2 \mathrm{~T}$ at the interaction point to about $1 \mathrm{~T}$ at $z=-3 \mathrm{~m}$. Simulations, shown for two values of $\theta$ in figure 43 , demonstrate that the momentum of backward

\footnotetext{
${ }^{10}$ This latter requirement necessitates the removal of one of the muon chambers in this region. The consequences of this for the physics performance of the $\mathrm{H} 1$ detector are still to be investigated. If they are severe, a new, smaller module can be built.
} 


\begin{tabular}{|c|c|}
\hline VBST z positions/m & $\begin{array}{lllll}-1.30 & -1.40 & -1.50 & -1.60 & -1.70\end{array}$ \\
\hline $\mathrm{rmin} / \mathrm{cm}$ & 10 \\
\hline $\mathrm{rmax} / \mathrm{cm}$ & 22 \\
\hline number of planes & 10 \\
\hline area of $\mathrm{Si}$ in $\mathrm{m}^{2}$ & 1.2 \\
\hline estimated cost in kEuro & 600 \\
\hline VVBST z positions/m & $\begin{array}{llllll}-1.85 & -2.00 & -2.15 & -2.30 & -2.45 & -2.60\end{array}$ \\
\hline $\mathrm{rmin} / \mathrm{cm}$ & 4 \\
\hline $\mathrm{rmax} / \mathrm{cm}$ & 16 \\
\hline number of planes & 12 \\
\hline area of $\mathrm{Si}$ in $\mathrm{m}^{2}$ & 0.9 \\
\hline estimated cost in kEuro & 450 \\
\hline
\end{tabular}

Table 5: Parameters of the new backward silicon tracker modules, made of $6^{\prime \prime}$ wafers, single sided and mounted back to back. The resolution required is about $15 \mu \mathrm{m}$ which can be achieved with, for example, a pitch of $25 \mu \mathrm{m}$ with every third strip being readout. An amplifier such as the radiation hard version of the APC128 [156] would be adequate for this purpose.

scattered tracks can be reconstructed with the necessary accuracy using the run vertex as a constraint. This is accurately determined by the CJC and the central silicon detectors. As has been done using the BST and the SpaCal [155], the VBES can be calibrated and the background to the high $y$ DIS sample determined. The background is removed by subtracting the "wrong charge" photoproduction events from the "lepton beam charge" events which consist of photoproduction background and the required high $y$ DIS events, see figure 44 . This allows reliable measurement of the DIS cross section up to the highest $y$ values, and thus the extraction of the longitudinal structure function.

The second very backward silicon tracker, the VVBST, requires an exit window in the beam pipe in order that the momentum measurements it makes are not spoiled by multiple scattering. Such windows have already been used at HERA, for example for the ZEUS BPC spectrometer.

\subsubsection{The very backward proportional chamber}

The requirements that there be redundancy in the backward tracking and that the VBES cluster position be accurately reconstructed as well as alignment considerations make it necessary to have a tracking chamber positioned immediately in front of the VBES calorimeter. Here, we propose that this chamber be similar to the present BPC. This has obvious advantages with regard to operation and software development. The chamber's parameters are summarised in table 6.

\subsubsection{The very backward electromagnetic SpaCal}

The VBES is composed of $5 \times 5$ supermodules, corresponding to $20 \times 20$ cells, each of lateral dimension $20 \times 4.05 \mathrm{~cm}^{2}$ and including 4 veto cells placed immediately around the beam-pipe. 


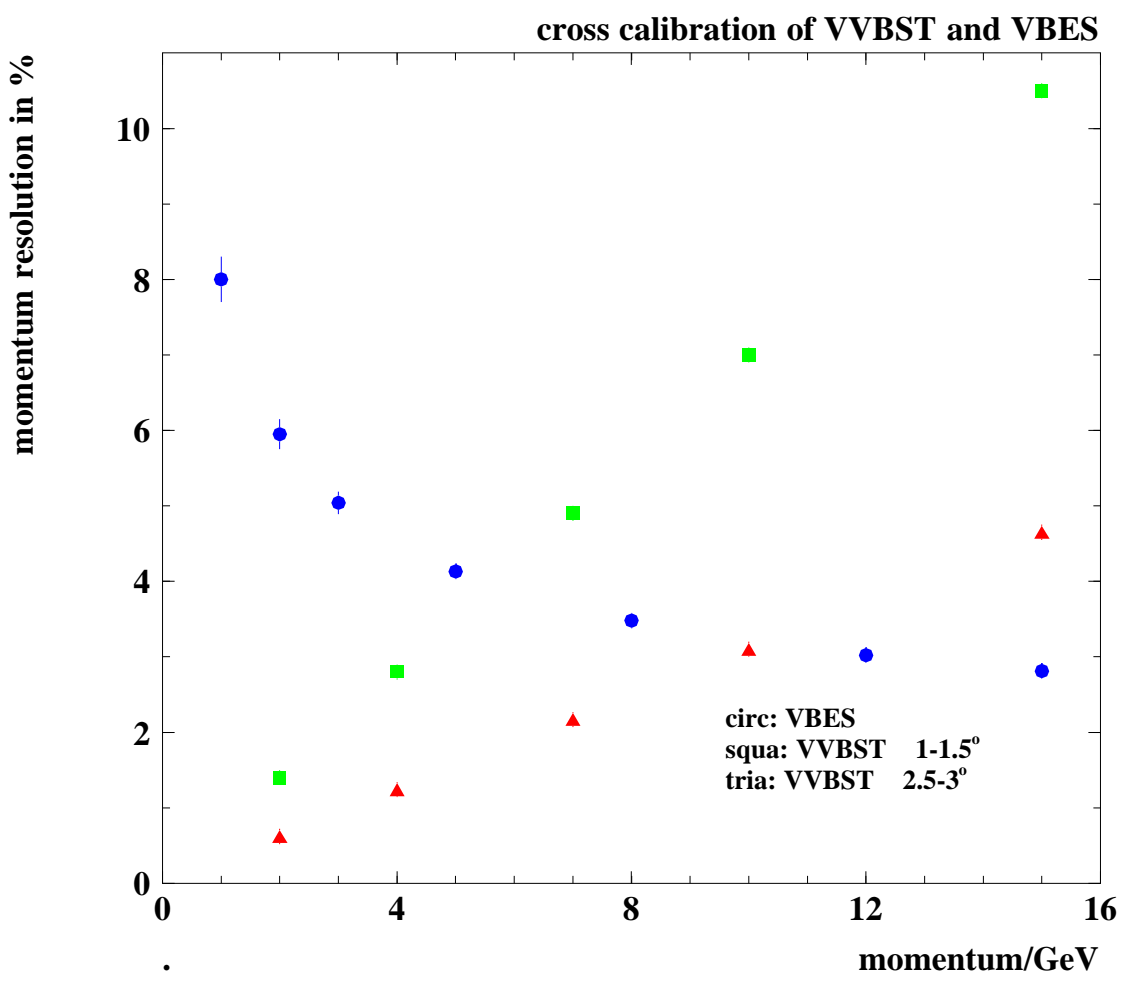

Figure 43: The main task of the silicon tracker is to measure the polar angle and the charge of scattered electrons. At low energies, depending on the polar scattering angle, the tracker measures the momentum with sufficient accuracy to allow the calibration of the VBES energy scale, as is done currently with the H1 BST/SpaCal detectors [157]. With exit windows as sketched in figure 41, the multiple scattering as the electrons traverse the beam pipe is negligible compared to that which occurs in the silicon planes.

\begin{tabular}{|l|c|}
\hline z position/m & -3.00 \\
square inner radius $(\mathrm{cm})$ & 5 \\
square outer radius $(\mathrm{cm})$ & 40 \\
number of wire planes & $3 \times 2$ \\
spatial point resolution $(\mu \mathrm{m})$ & 250 \\
estimated cost (kEuro) & 100 \\
\hline
\end{tabular}

Table 6: Parameters of the very backward proportional chamber (VBPC) which is attached to the VBES. 


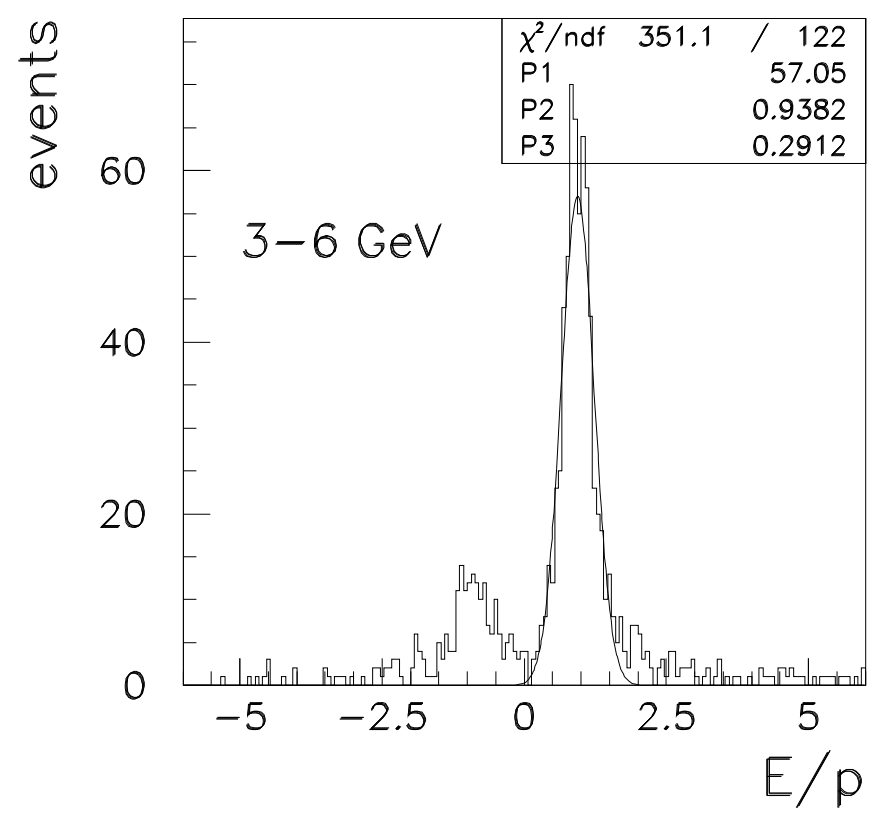

Figure 44: The ratio $E / p$ of the energy $E$ measured in the SpaCal and the momentum $p$ measured in the current BST. The small peak at negative $p$ is the wrong charge background while the large peak comprises both the dominant DIS signal and the photoproduction background due to particles with the same charge as the beam positrons.

Its total size is $81 \times 81 \mathrm{~cm}^{2}$ and total weight 1.2 tonnes. The latter is much less than the 6.8 tonne weight of the compensator coil which was supported at approximately the same position during HERA I running and therefore does not present a problem. Two options for supporting the VBES are possible:

1. Construct the VBES in two halves, split vertically, and fix these directly to the iron shells (north/south). The position reproducibility of the iron shells is better than $\sim 1 \mathrm{~mm}$. A precise optical position monitoring system e.g. as used in the H1 Roman pots could be installed to measure the shell positions with an accuracy reaching $\sim 10 \mu \mathrm{m}$.

2. Support the VBES on a long lever arm from the wall or magnet bridge, as was done for the compensator coil.

The number of new modules etc. that must be fabricated for the VBES is limited. Assuming that nine SpaCal electromagnetic supermodules can be reused, 16 new supermodules, with a total of 256 new electronics channels will be required. A number of useable supermodules are still available from the mass production of the electromagnetic SpaCal with additional free lead sheets. Moreover, the rolling machine is still available, and can be used for the production of more lead sheets. The number of required new channels fits into two new analogue boxes which would have to be equipped with 16 front-end analogue cards. The approximate cost of the VBES is given in table 7. 
The possible effect of any non-uniformity of the magnetic field on the operation of the Hamamatsu R-5505 photomultipliers requires more investigation. Small distortions of the magnetic field leading to limited gain losses can be compensated for by the application of higher voltages.

Due to the increased separation in time of electrons and protons/deuterons in the VBES, the veto function of the present backward time-of-flight system could be taken over by a VBES trigger.

\begin{tabular}{|l|r|}
\hline 300 Hamamatsu PMT's for VBES & 150 kEuro \\
\hline 2 Wiener analog boxes power supplies for VBES & 30 kEuro \\
\hline 20 front-end and 20 analog cards for VBES & 100 kEuro \\
\hline fibres and lead & 30 kEuro \\
\hline
\end{tabular}

Table 7: Estimated cost for the new very backward Spacal

\subsubsection{Backward spectrometer summary}

A new backward spectrometer has been sketched which enables DIS to be measured with high precision in the transition region of momentum transfers $Q^{2}$ between 0.2 and $10 \mathrm{GeV}^{2}$. The spectrometer is based on $\mathrm{H} 1$ experience with silicon tracking and SpaCal calorimetry supported by a proportional chamber. This system can be realised within $2 \ldots 3$ years of approval for an estimated cost of 1.6 MEuro. Such a system is essential for any future attempt to access the low $x$ region with the $\mathrm{H} 1$ detector after completion of its high luminosity programme.

\section{Future HERA operation}

\subsection{Introduction}

This section of the report describes briefly some of the modifications that will have to be made to the HERA accelerator complex if it is to provide the beams needed for the electron-deuteron, electron-ion and spin programmes described above.

\subsection{Deuterons in HERA}

Since deuterons have about twice the mass of protons, they have a lower speed than protons of the same kinetic energy. Therefore the acceleration of deuterons to, and storage at, high energy in HERA would require some modifications to the very low energy part of the pre-accelerator chain: from the radio frequency quadrupole (RFQ) through the LINAC-III and into DESY-III. 
- Deuterons would be accelerated in LINAC-III in the so called " $2 \beta \lambda$ " mode whereby deuterons enter the linac with about half of the speed that the protons normally have and "see" a gap voltage of half of the standard so that the deuterons would remain at half the standard speed for the whole length. The linac would be run at the standard frequency and the deuterons would see only every second available kick in the accelerating gaps between the drift tubes. Thus they would emerge with $25 \mathrm{MeV}$ kinetic energy instead of the $50 \mathrm{MeV}$ of protons. In this way, LINAC-III would need no modifications.

- A new dedicated RFQ would be needed and it should produce the required speed for the linac.

- At $25 \mathrm{MeV}$ the circulation time for deuterons in DESY-III is about $6 \mu$ sec instead of the $3 \mu \mathrm{sec}$ for $50 \mathrm{MeV}$ protons. At $7.5 \mathrm{GeV} / \mathrm{c}$ the circulation time is about $1 \mu \mathrm{sec}$. So for deuterons the radio frequency accelerating system in DESY-III would need to be modified to give a frequency range of six instead of three.

- The crossing of the "transition" energy in HERA would need to be handled.

- Enhanced space charge and intra-beam scattering effects would have to be accommodated.

Estimates of the luminosity achievable in $e d$ running suggest it will be approximately half of that attained in $e p$ collisions at HERA.

\subsection{Ions in HERA}

The last of the difficulties mentioned above for the storing, acceleration and collision of electrons and deuterons in HERA becomes increasingly problematic as the charge of the ions involved increases. The ion beam lifetime therefore decreases rapidly with increasing atomic mass and operation of HERA with ions heavier than deuterium would require the introduction of ion cooling schemes. Electron cooling in the final stage of the injector chain and in HERA itself would allow respectable luminosities to be achieved with ions as heavy as carbon and oxygen [158].

Recently, an alternative cooling scheme has been proposed that relies on the excitation of the accelerated nuclei by laser light [159]. The frequency of the laser must match the energy of a nuclear transition in the ions of the beam, once allowance has been made for the Lorentz transformation to the frame of reference of the beam particles. More energetic nuclei are favourably excited by the light due to the doppler effect and this, together with the scanning of the laser frequency from lower to higher values, leads to the required cooling. Because of the necessary matching of the laser frequency and the nuclear transitions, only particular pairs of lasers and nuclei are suitable for this cooling scheme. It would be possible to construct a laser based on FEL technology, similar to the TTF laser, which matches the energy of a nuclear transition in mercury. This raises the intriguing possibility that HERA could be used for the collision of electrons with ${ }^{201} \mathrm{Hg}$ ions [159]. 


\subsection{Polarised protons and deuterons in HERA}

If the HERA acceleration chain is to be upgraded to handle deuterons, then serious consideration should be given to ensuring that spin polarised deuterons can also be provided. DESY is already expert in the theory, phenomenology and practice of high energy, highly longitudinally polarised electron and positron beams in the HERA electron ring. Moreover, members of the HERA accelerator group have devoted several years to an extensive study of obtaining polarised protons at high energy in HERA [160-165]. Whereas electron polarisation occurs naturally as the result of synchrotron radiation emission, polarised protons must be provided from a source and then accelerated. Thus that study covered the maintenance of polarisation in all accelerators of the acceleration chain. Moreover, owing to the very high energy $(920 \mathrm{GeV})$ to be reached it was necessary to develop very significant extensions to the conceptual basis, both mathematical and physical, for the understanding of depolarising effects during acceleration. These concepts were then exploited in the large and sophisticated computer code SPRINT which was specially written for this study $[164,165]$. The provision of polarised protons at high energy would entail a large effort. However, as explained below, the provision of polarised deuterons would probably entail less effort and would be a natural development for DESY. In this case too, the deuterons would have to be polarised already at the source. An unpolarised deuteron beam has already been stored at $100 \mathrm{GeV}$ in the Relativistic Heavy Ion Collider (RHIC) at Brookhaven National Laboratory (BNL) [166]. Since deuterons are fragile, it is important to know whether they dissociate during collision with the electromagnetic fields of the oncoming beam (beambeam interaction). In principle a first impression of the level of dissociation can be deduced from measurements of deuteron beam lifetime in RHIC.

There are four basic elements in the provision of high energy polarised deuterons:

- a source of polarised deuterons:

- a way of maintaining the polarisation during acceleration:

- a way to manipulate the direction of the polarisation at the interaction points at the top energy:

- polarimeters to monitor the polarisation at each accelerator in the chain and provide information for optimising the chain.

\subsubsection{The source}

In analogy with the case of polarised protons, a source capable of delivering $20 \mathrm{~mA}$ pulses of highly polarised $D^{-}$atoms would be needed. But for deuterons the ten turn injection through the stripping foil would need $66 \mu$ sec pulses instead of the $33 \mu$ sec pulses of protons. As with protons, invariant emittances of $2 \pi \mathrm{mm}$ mrad would be needed. 


\subsubsection{Maintaining polarisation during acceleration}

The central problem for the acceleration of any polarised beam in a circular accelerator is the coupling of the spins to the magnetic guide fields. If the particles were only confronted with the vertical bending fields, then vertical spins could be accelerated without problems. However, the particles must be contained using quadrupole fields too. Then the vertical spins are tilted away from the vertical by the radial fields in the quadrupoles and tilted spins precess around the vertical bending fields. These combined influences can lead to very strong disturbance of the spins and to a potential decrease of the polarisation whenever the spin-orbit resonance condition $\nu_{\text {spin }}=m_{0}+m_{x} \cdot Q_{x}+m_{z} \cdot Q_{z}+m_{s} \cdot Q_{s}$ is satisfied. In this expression the $m_{0, x, z, s}$ are integers, the $Q_{x, z, s}$ are the "tunes" of the horizontal, vertical and longitudinal motion, and $\nu_{\text {spin }}$ is the "spin tune", i.e. the number of times per turn that a spin precesses around the vertical in the bending fields. In a flat ring with no solenoid fields, $\nu_{\text {spin }}=a \gamma$ where $a$ is the gyromagnetic anomaly and $\gamma$ is the Lorentz factor. Since $\nu_{\text {spin }}$ is proportional to energy, the beam can cross the spin-orbit resonance condition many times before the top energy is reached.

\subsubsection{Protons}

For protons, $a \approx 1.7928$ so that $\nu_{\text {spin }}$ increments by one for every $523 \mathrm{MeV}$ increase in energy. Thus on the way to $920 \mathrm{GeV}$ thousands of resonances are crossed and without recourse to special measures the polarisation would be lost. The primary measure for protons in PETRA and HERA is to introduce magnet systems called "Siberian Snakes" which have the effect of fixing $\nu_{\text {spin }}$ to be essentially independent of energy and close to $1 / 2$. Thus, by suitable choice of orbital tunes, resonance crossing and depolarisation can be avoided during acceleration. Siberian Snakes would cause too much orbit distortion in DESY-III so that alternative strategies are needed. In particular, for the so called imperfection resonances $\left(\nu_{\text {spin }}=m_{0}\right)$, "partial snakes" would be used. These deliberately reverse the direction of the polarisation at resonance crossing while preserving its value. An analogous approach would be used at the next most important resonances, namely the first order vertical intrinsic resonances $\left(\nu_{\text {spin }}=m_{0} \pm Q_{z}\right)$. In that case the vertical orbital amplitude would be temporarily increased by applying a radial radio frequency field close to resonance with the vertical betatron motion. The use of Siberian Snakes to maintain proton polarisation up to high energy, namely up to $100 \mathrm{GeV} / \mathrm{c}$ in RHIC, was demonstrated in December 2001 [167].

\subsubsection{Deuterons}

In contrast to protons, for deuterons $a \approx-0.143$. In addition the deuteron mass is about twice the proton mass so that at the same momentum $\gamma$ is about half of that for protons. Thus $a \gamma$ increments by one for every $\approx 13 \mathrm{GeV}$ increase in energy. As a result, deuteron spins would be subject to 25 times fewer resonances on the way to the top energy. Moreover, the strength of the coupling of the spins to the magnetic fields is a factor of 25 smaller than the for protons so that the so called "resonance strengths" which quantify the influence of resonance crossing on the polarisation, are of the order of 25 times smaller, as shown in figure 45 . 


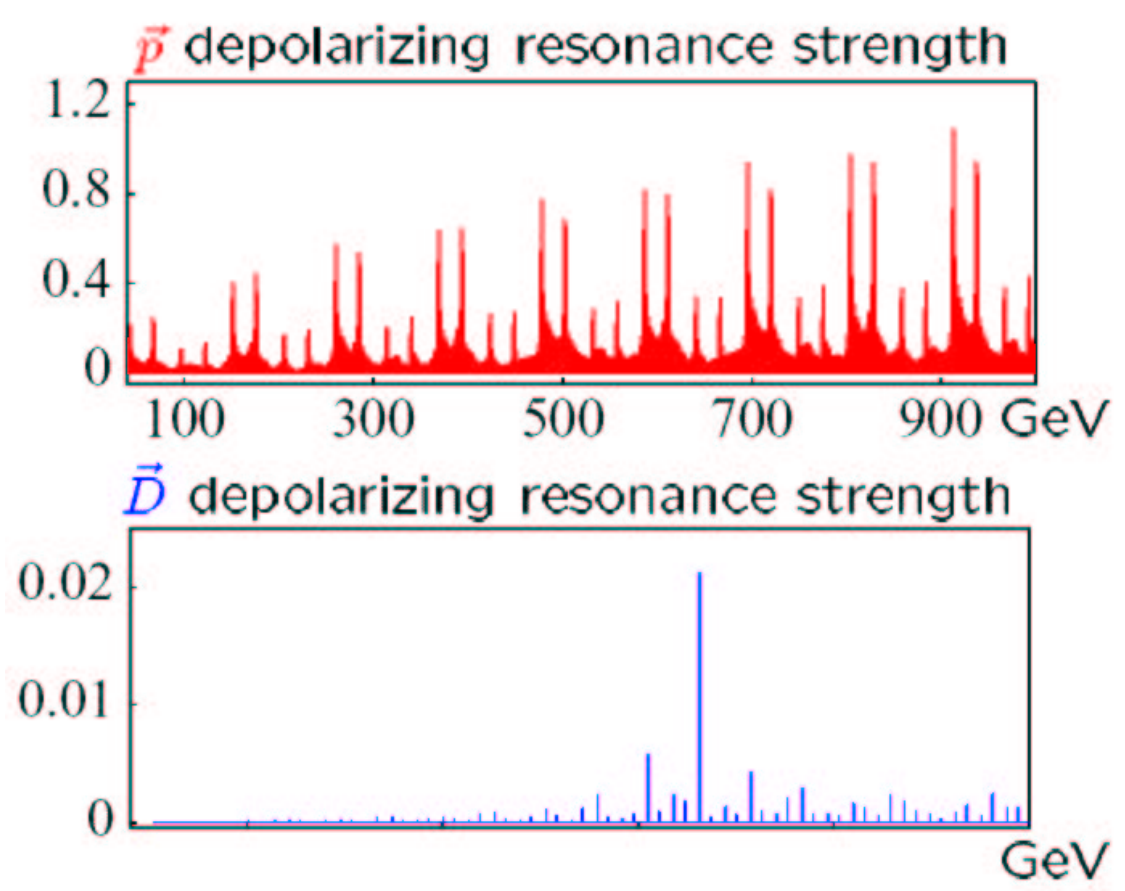

Figure 45: A comparison of the resonance strengths for protons and deuterons in the HERA proton ring [168]

In summary: for deuterons there are far fewer and far weaker resonances than for protons. This gives grounds for optimism that the preservation of vertical deuteron polarisation during acceleration to the top energy would be much more practical and straightforward than for protons. Nevertheless, since the resonance strengths are not zero, some depolarisation must be expected. But given the low number of resonances, there might be scope for energy dependent "spin matching", i.e. adjusting the optic so as to minimise the depolarisation as troublesome resonances are crossed. In any case, the level of depolarisation must be estimated by careful and extensive spin-orbit tracking simulations using SPRINT. Note that the interleaved vertical and horizontal bends on each side of the North, South and East interaction points represent significant magnetic field imperfections for the spin motion but might in fact be put to use as partial snakes and thereby serve to overcome depolarisation. The presence of the vertical bends would also mean that the polarisation would in general not be exactly vertical in the arcs or at the interaction points. However, the direction of the polarisation could be precisely calculated and the energy chosen so as to minimise the tilt at the interaction points.

\subsubsection{Manipulating deuteron polarisation at the top energy}

A complete experimental programme with polarised deuterons would involve longitudinal polarisation as well as transverse polarisation. In the case of protons this could be achieved by means of spin rotators, i.e. special magnet systems which rotate the polarisation to longitudinal just before an interaction point and then back to the vertical after the interaction point. 
Such rotators would be about $10 \mathrm{~m}$ long and have fields of about $4 \mathrm{~T}$ as at RHIC. However, for deuterons the weakness of the spin orbit coupling, which has advantages for preserving the polarisation during acceleration, makes it impossible to construct practical spin rotators: the field integral would have to be about 25 times larger than for protons. For the same reason, practical Siberian Snakes cannot be built for deuterons although, as we have implied, they would probably not be needed. However, it has been suggested $[169,170]$ that spin rotation could be achieved by applying an external horizontal radio frequency field with a frequency chosen close to resonance with the natural spin precession, in particular close to resonance with the fractional part of $\nu_{\text {spin }}=a \gamma$. This would be analogous to manipulation of spins by spin resonance in condensed matter physics. Once the beam had reached full energy, the radio frequency field would be switched on and its frequency would be swept to the resonance, thereby adiabatically dragging the polarisation to the direction of the external field at the field source. By choosing a suitable energy and a suitable position (or direction) for the external field, the polarisation would be longitudinal at one interaction point. Examples where a radio frequency field has already been used for manipulating, in fact flipping, deuteron spins are mentioned below. The use of such fields is common practice for depolarising electron (positron) beams and for flipping the polarisation of proton beams. The next step is to check the feasibility of the suggestion in $[169,170]$ by carrying out extensive and realistic spin-orbit tracking simulations for very high energy deuterons.

Beam-beam forces can have a profound effect on beam stability and polarisation. This must be investigated with SPRINT.

\subsubsection{Manipulating deuteron polarisation in LINAC-III, DESY-II and PETRA}

There would be no depolarisation in LINAC-III and it is expected that for the limited and relatively low energies of DESY-III and PETRA, resonant depolarisation would not be a serious problem. But, as for the HERA ring itself, this must be checked by extensive spin-orbit tracking. If it is needed, spin matching might be feasible in these rings too. Polarised deuteron beams were accelerated to $12 \mathrm{GeV}$ in the Argonne ZGS [171], and to $2.3 \mathrm{GeV}$ in Saturn (Saclay) [172]. A polarised deuteron beam has been accelerated to $9 \mathrm{GeV} / \mathrm{c}$ at JINR, Dubna [173] and deuteron polarisation has been maintained up to $10.2 \mathrm{GeV}$ in the KEK-PS [174]. Deuteron spins have been flipped at very low energy in the IUCF ring [175]. Polarised deuterons can now be stored in the COSY ring at Juelich at $1.85 \mathrm{GeV} / \mathrm{c}$ [176]. Deuteron spin flip has also been achieved in COSY.

\subsubsection{Polarimetry}

In addition to the polarimeter needed to provide absolute polarisation measurements at the top energy, polarimeters are also needed to monitor the polarisation at each accelerator in the chain and provide information for optimising that chain. However, these latter need not provide absolute measurements; relative measurements would suffice. At the KEK-PS and at Dubna the vector polarisation is measured by observing elastic scattering on carbon. Further work is needed to develop polarimeters for very high energy, see also the discussion in [177]. Polarimeters for both vector and tensor polarisation would be needed. 


\section{Summary}

Since its discovery thirty years ago, Quantum Chromo-Dynamics has proven to be an enormously rich theory that continues to excite the interest of theoretical and experimental physicists alike. One of the most powerful experimental techniques for the study of QCD is deep inelastic scattering, and the preeminent facility for DIS is the HERA collider at DESY. HERA is currently engaged in investigations of the highest energy lepton-proton collisions ever achieved in the laboratory, allowing the probing of proton structure down to distances of $\frac{1}{1000}^{\text {th }}$ of the size of the proton and the search for physics linking the electroweak and strong sectors of the Standard Model (SM). Following the completion of the current HERA programme, the HERA collider and the H1 experiment can be used to investigate some of the outstanding problems of QCD. These include:

- Mapping the parton distributions of the nucleon and understanding the constraints, for example due to non-perturbative effects, that influence those distributions.

- Precise measurement of the strong coupling constant, the least well known of the fundamental constants of nature.

- Studying effects such as diffraction and shadowing and the links between them.

- Investigating QCD at high parton densities in nuclei.

- Studying colour radiation processes over the full rapidity range available at HERA.

- Developing a 3D picture of nucleon structure via the study of deeply virtual Compton scattering and related processes.

- Understanding the partonic origins of nucleon spin.

This Letter of Intent proposes that HERA should be used to collide electrons and positrons with deuterons in order that the first points above can be addressed. The Letter describes the suitability of $\mathrm{H} 1$ as a detector for this programme and proposes upgrades where these are needed.

Extensions into the forward and backward regions and the ability to measure DIS with high precision open an exciting perspective for the $\mathrm{H} 1$ experiment beyond its current phase, including measurements of parton interactions at high density and the exploration of spin in an unknown kinematic domain.

\section{References}

[1] K. Hagiwara et al. (Particle Data Group) Phys. Rev. D66 010001 (2002).

[2] P. Amaudruz et al. [NMC Collaboration] Phys. Rev. Lett. 662712 (1991).

[3] K. Gottfried, Phys. Rev. Lett. 181174 (1967). 
[4] A. Baldit et al. [NA51 Collaboration] Phys. Lett. B332 244 (1994).

[5] J.C. Peng et al. [NuSea Collaboration] Phys. Rev. D58 092004 (1998);

R.S. Towell et al. Phys. Rev. D64 052002 (2001).

[6] K. Saito, M. Ueda, K. Tsushima and A.W. Thomas, Eur. Phys. J. A12 257 (2001).

[7] R.P. Feynman and R.D. Field, Phys. Rev. D15 2590 (1977);

S. Kumano, Phys. Rep. D303 183 (1998).

[8] B.Z. Kopeliovich, B. Povh and A. Schäfer, unpublished, February 2003.

[9] D. Diakonov, V. Petrov, P. Pobylitsa, M.V. Polyakov and C. Weiss, Nucl. Phys. B480 341 (1996) [hep-ph/9606314];

K. Tsushima, A.W. Thomas and V. Dunne, hep-ph/0107042;

R. Jackiw and C. Rebbi, Phys. Rev. D13 3398 (1976);

see also R. Jackiw, hep-th/9903155, Dirac prize lecture, Trieste (1999).

[10] K. Saito, Eur. Phys. J. A12 257 (2001).

[11] H1 Collaboration, C. Adloff et al., hep-ex/0304003, Submitted to Eur. Phys. J.

[12] M. Dittmar, F. Pauss and D. Zürcher, Phys. Rev. D56 11 (1997).

[13] J. Pumplin et al. (CTEQ Collaboration) JHEP 0207012 (2002) [arXiv:hep-ph/0201195].

[14] C. Spiering, arXiv:astro-ph/0303068:

F. Halzen, arXiv:astro-ph/0301143 v1 (2003).

[15] M. Gluck, S. Kretzer and E. Reya, Astropart. Phys. 11327 (1999) [arXiv:astro$\mathrm{ph} / 9809273]$.

[16] K. Giesel, J.H. Jureit and E. Reya, arXiv:astro-ph/0303252 and ref.s therein.

[17] D.A. Dicus, S. Kretzer, W.W. Repko and C. Schmidt, Phys. Lett. B514 103 (2001) [arXiv:hep-ph/0103207].

[18] M. Gluck, E. Reya, M. Stratmann and W. Vogelsang, Phys. Rev. D63 094005 (2001) [arXiv:hep-ph/0011215].

[19] M. Klein, Nucl. Phys. Proc. Suppl. B116 91 (2003).

[20] C. Adloff et al. (H1 Collaboration), Eur. Phys. J. C21 33 (2001) [arXiv:hep-ex/0012053].

[21] V.N. Gribov, Zh. Exp. Teor. Fiz. 571309 (1969);

Sov. Phys. JETP 30709 (1970).

[22] L. Frankfurt, V. Guzey and M. Strikman, hep-ph/0304149.

[23] J. Webb et al. (E866/NuSea Collaboration) hep-ex/0302019.

[24] A. Bodek and U.K. Yang, Phys. Rev. Lett. 822467 (1999) and hep-ex/0301036. 
[25] S. Kuhlmann et al. Phys. Lett. B476 291 (2000) and hep-ph/0007141.

[26] A.C. Benvenuti et al. Phys. Lett. B237 592 (1990);

ibid. 599.

[27] L.W. Whitlow et al. Phys. Lett. B282 475 (1992).

[28] W. Melnitchouk and A.W. Thomas, arXiv:nucl-th/0207056, see also Phys. Lett. B377 11 (1996).

[29] G. Dodge et al. (CLAS Collaboration) Experiment E-03-012 Jefferson Lab.

[30] A.H. Mueller, arXiv:hep-ph/0208278 and hep-ph/0301109 and Ref.s cited therein.

[31] V.S. Fadin, V.T. Kim, L.N. Lipatov and G.B. Pivovarov, arXiv:hep-ph/0207296 and Ref.s cited therein.

[32] B. Andersson et al. (Small x Collaboration), Eur. Phys. J. C25 77 (2002) [arXiv:hep$\mathrm{ph} / 0204115]$.

[33] M. Ciafaloni, D. Colferai and G.P. Salam, Phys. Rev. D60 114036 (1999) [arXiv:hep$\mathrm{ph} / 9905566]$.

[34] J.A. Vermaseren, S. Moch and A. Vogt, arXiv:hep-ph/0211296 in Nucl. Phys. B, Proc. Suppl. 116 (2003).

[35] F.J. Yndurain, Nucl. Phys. Proc. Suppl. 114 (2003) 95 [arXiv:hep-ph/0202020].

[36] P. Langacker, arXiv:hep-ph/0304093.

[37] S. Chekanov et al. (ZEUS Collaboration) Phys. Rev. D67 012007 (2003) [arXiv:hepex/0208023].

[38] A. Vogt, Proc. DIS 1996, Rome, Italy.

[39] For a very thorough investigation see R. Wallny, Ph.D. thesis Zürich 2001, see http://wwwh1.desy.de/publications/theses_list.html

[40] M. Klein, A. Tapper and Wu-Ki Tung, Talks at the DESY Forum on Parton Distributions and QCD, March 2003, unpublished, see http://www.desy.de/f/seminar/sem_schedule.html.

[41] M. Botje, M. Klein and C. Pascaud, Proc. HERA Workshop 1996, p. 33, Ed.s G. Ingelman, A. De Roeck and R. Klanner.

[42] S.J. Brodsky, arXiv:hep-ph/0208158.

[43] V.N. Gribov, Sov. Phys. JETP 29483 (1969) [Zh. Eksp. Tor. Fiz. 56892 (1969)].

[44] V.A. Abramovsky, V.N. Gribov and O.V. Kancheli, Yad. Fiz. 18595 (1973).

[45] L. Alvero, L. Frankfurt and M. Strikman, Eur. Phys. J. A5 97 (1999). 
[46] J.C. Collins, Phys. Rev. D57 3051 (1998).

[47] L. Frankfurt and M. Strikman, Eur. Phys. J. A5 293 (1999).

[48] L. Frankfurt, V. Guzey, M. McDermott and M. Strikman, J. High Energy Phys. 202 (2002) 27.

[49] L. Frankfurt, V. Guzey and M. Strikman, "Leading twist nuclear shadowing: a user's guide", preprint hep-ph/0303022.

[50] C. Adloff et al. (H1 Collaboration) Z. Phys. C76 613 (1997).

[51] R.J. Glauber, Phys. Rev. 100242 (1955).

[52] V. Gribov, Sov. Phys. JETP 30709 (1970).

[53] L. Frankfurt, V. Guzey, M. McDermott, M. Strikman, Phys. Rev. Lett. 87192301 (2001).

[54] H1 Collaboration, VFPS Proposal, H1-5/00-582;

P. Van Mechelen, A Very Forward Proton Spectrometer for H1, [hep-ex/0203029].

[55] e.g. A. Carroll et al. Phys. Lett. B80 423 (1979).

[56] e.g. A. Belousov et al. Yad. Fiz. 21556 (1975);

D. Caldwell et al. Phys. Rev. D7 1362 (1973).

[57] P. Newman, "Prospects for Diffraction at HERA II", in Proc. of International Workshop on Deep Inelastic Scattering, DIS02, Cracow.

[58] A. Mehta, J. Phillips, B. Waugh, "Future Physics at HERA", Proc. Workshop, DESY 1996, Ed.s G. Ingelman, A. De Roeck and R. Klanner, p. 704 (http://www.desy.de/ heraws96).

[59] L. Frankfurt, G. Piller, M. Sargsian and M. Strikman, Eur. Phys. J. A2 301 (1998) [arXiv:nucl-th/9801041].

[60] L. Hulthen and M. Sugawara, Handbuch der Physik, 39 (1957) 1;

G. Alberi, L. Bertocci and G. Biakowski, Nucl. Phys. 17621 (1970);

M. Lacombe et al. Phys. Lett. B101 139 (1981).

[61] CȦdloff et al. (H1 Collaboration) Phys. Lett. B520 183 (2001).

[62] V. Gribov and L. Lipatov, Sov. J. Nucl. Phys. 15438 (1972); ibid. 675.

[63] L. Lipatov, Sov. J. Nucl. Phys. 2094 (1975).

[64] G. Altarelli and G. Parisi, Nucl. Phys. B126 298 (1977).

[65] Y. Dokshitser, Sov. Phys. JETP 46641 (1977).

[66] J. Rosner, Proc. Adv. Study Inst. on Techniques and Concepts in HEP, St. Croix, USVI, Ed. T. Ferbel, 1980. 
[67] K. Prytz, Phys. Lett. B311 286 (1993).

[68] E. Iancu, A. Leonidov, L. McLerran, hep-ph/0206073.

[69] J. Blümlein et al. hep-ph/0102025 (2001).

[70] G. Altarelli, R.D. Ball and S. Forte, Nucl. Phys. B575 313 (2000).

[71] A.D. Martin, R.G. Roberts, W.J. Stirling and R.S. Thorne, Phys. Lett. B531 216 (2002) [arXiv:hep-ph/0201127];

see also R.S. Thorne, talk at the HERA III workshop at MPI Munich, Dec. 2002, unpublished, see http://wwwhera-b.mppmu.mpg.de/hera3/hera3.html.

[72] C. Callan and D.J. Gross, Phys. Rev. Lett. 22156 (1969).

[73] A. Zee, F. Wilczek and S.B. Treiman, Phys. Rev. D10 2881 (1974);

G. Altarelli and G. Martinelli, Phys. Lett. B76 89 (1978);

M. Glück and E. Reya, Nucl. Phys. B145 24 (1978).

[74] J. Bartels, K. Golec-Bienat and J. Peters, Eur. Phys. J. C17 171 (2000);

E. Gotsman it et al. hep-ph/00081280 (2000).

[75] L. Bauerdick, A. Glazov and M. Klein, hep-ex/9609017 (1996), Proc. HERA physics workshop, Ed. G. Ingelmann, A. DeRoeck and R. Klanner, DESY 1996, p. 77.

[76] C. Adloff et al. (H1 Collaboration), Phys. Lett. 393B 452 (1997);

C. Adloff et al. (H1 Collaboration), Eur. Phys. J. C21 33 (2001), hep-ex/0012053;

A New Measurement of the Deep Inelastic Scattering Cross Section and of $F_{L}$ at Low $Q^{2}$ and Bjorken- $x$ at HERA, paper 799, sub. to EPS 2001, Budapest.

[77] E. Kuraev, L. Lipatov and V. Fadin, Sov. Phys. JETP 44443 (1976).

[78] E. Kuraev, L. Lipatov and V. Fadin, Sov. Phys. JETP 45199 (1977).

[79] Y. Balitski and L. Lipatov, Sov. J. Nucl. Phys. 28822 (1978).

[80] M. Ciafaloni, Nucl. Phys. B 296 (1988) 49.

[81] S. Catani, F. Fiorani, G. Marchesini, Phys. Lett. B 234 (1990) 339.

[82] S. Catani, F. Fiorani, G. Marchesini, Nucl. Phys. B 336 (1990) 18.

[83] G. Marchesini, Nucl. Phys. B 445 (1995) 49.

[84] B. Andersson, G. Gustafson, J. Samuelsson, Nucl. Phys. B 467 (1996) 443.

[85] B. Andersson, G. Gustafson, H. Kharraziha, J. Samuelsson, Z. Phys. C 71 (1996) 613.

[86] G. Gustafson, H. Kharraziha, L. Lönnblad, The LCD Event Generator, in Proc. of the Workshop on Future Physics at HERA, edited by A. De Roeck, G. Ingelman, R. Klanner (1996), p. 620.

[87] H. Kharraziha, L. Lönnblad, JHEP 03 (1998) 006. 
[88] G. 't Hooft, Nucl. Phys. B190 (1981) 455.

[89] J.D. Bjorken, K.L. Kowalski, C.C. Taylor, (1993) .

[90] B. Andersson, G. Gustafson, J. Samuelsson, Zeit. Phys. C64 (1994) 653.

[91] T. Sjostrand et al. Comput. Phys. Commun. 135 (2001) 238, hep-ph/0010017.

[92] G. Marchesini et al. Comp. Phys. Comm. 76 (1992) 465, hep-ph/9912396.

[93] A. Mueller, Nucl. Phys. B (Proc. Suppl.) 18C (1990) 125.

[94] A. Mueller, J. Phys. G 17 (1991) 1443.

[95] C. Adloff et al. (H1 Collaboration) Nucl. Phys. B 538 (1999) 3.

[96] J. Breitweg et al. (H1 Collaboration) Eur. Phys. J. C 6 (1999) 239.

[97] H. Jung and G. Salam, Eur. Phys. J. C 19 (2001) 351, hep-ph/0012143.

[98] J.G. Contreras, Phys. Lett. B446 158 (1999).

[99] J.G. Contreras, R. Peschanski and C. Royon, Phys. Rev. D62 034006 (2000).

[100] L. Lönnblad, Comp. Phys. Comm. 71 (1992) 15.

[101] N. Brook et al. "A comparison of deep-inelastic Monte Carlo event generators", in Proceedings of the Workshop on Monte Carlo generators for HERA physics, edited by A. Doyle, G. Grindhammer, G. Ingelman and H. Jung (DESY, Hamburg, 1999), p. 10.

[102] G. Ingelman, A. Edin and J. Rathsman, Comp. Phys. Comm. 101 (1997) 108.

[103] H. Jung, Comp. Phys. Comm. 143 (2002) 100, hep-ph/0109102, DESY 01-114, http : / / www . quark

[104] G. Ingelman, A. Edin, R. Enberg, J. Rathsman and N. Timneanu, Nucl. Phys. A663 1007 (2000).

[105] J.C. Collins, L. Frankfurt and M. Strikman, Phys. Rev. D56 2982 (1997).

[106] X. Ji, Phys. Rev. D55 7114 (1997).

[107] A.V. Radyushkin, Phys. Rev. D58 114008 (1998).

[108] M. Burkardt, Int. J. Mod. Phys. A18, 173 (2003);

Phys. Rev. D66 114005 (2002).

[109] L. Frankfurt and M. Strikman, Phys. Rev. D66 031502 (2002).

[110] L. Frankfurt, W. Koepf and M. Strikman, Phys. Rev. D57, 512 (1998)

[111] M. Vanderhaeghen, P.A. Guichon and M. Guidal, Phys. Rev. D60 094017 (1999).

[112] A. Freund, M. McDermott and M. Strikman, Phys. Rev. D67 036001 (2003). 
[113] A.H. Mueller, Nucl. Phys. B335 115 (1990).

[114] L. McLerran and R. Venugopalan, Phys. Rev. D50 225 (1994); Phys. Rev. D59 094002 (1999).

[115] E. Iancu, A. Leonidov and L. McLerran, hep-ph/0011241.

[116] M. Arneodo, A. Bialas, M.W. Krasny, T. Sloan and M. Strikman, "Nuclear beams in HERA,”,In *Hamburg 1995/96, Future physics at HERA* 887-926, hep-ph/9610423.

[117] R. Holt et al. Electron-Ion Collider White Paper, BNL 68933, February 2003.

[118] L. Frankfurt, V. Guzey, M. McDermott and M. Strikman, JHEP 0202, 027 (2002) [arXiv:hep-ph/0201230];

L. Frankfurt, V. Guzey and M. Strikman, Leading twist nuclear shadowing: a user's guide, preprint hep-ph/0303022.

[119] K.J. Eskola, V.J. Kolhinen and P.V. Ruuskanen, Nucl. Phys. B 535351 (1998);

K.J. Eskola, V.J. Kolhinen and C.A. Salgado, Eur. Phys. J. C9 61 (1999).

[120] L. Frankfurt, V. Guzey, M. McDermott and M. Strikman, Phys. Rev. Lett. 87, 192301 (2001) [arXiv:hep-ph/0104154, hep-ph/0104252].

[121] N.N. Nikolaev, B.G. Zakharov and V.R. Zoller, Z. Phys A35 435 (1995).

[122] L. Frankfurt and M. Strikman, Phys. Lett. B382 6 (1996).

[123] E. Gotsman, E. Levin, M. Lublinsky, U. Maor and K. Tuchin, Phys. Lett. B492, 47 (2000) [hep-ph/9911270].

[124] L. Frankfurt, M. Strikman and M. Zhalov, Phys. Lett. B537, 51 (2002) [arXiv:hep$\mathrm{ph} / 0204175]$.

[125] M. Diehl, Z. Phys. C66 181 (1995) [arXiv:hep-ph/9407399].

[126] B.»Denby et al. Phys. Rev. Lett. 52, 795 (1984).

[127] J. Ashman et al. Phys. Lett. B206 364 (1988);

Nucl. Phys. B328 1 (1989).

[128] U. Stoesslein, Int. J. Mod. Phys. A17 3220 (2002) [arXiv:hep-ex/0201036].

[129] "Prospects for Spin Physics", Proceeding of Workshop, Ed.s J. Blümlein and W.D. Nowak, DESY 95-200, Zeuthen 1995;

"Physics with polarized protons at HERA", Proceedings of Workshop, Ed.s A. De Roeck and T. Gehrmann, DESY-PROC-1998-01, Hamburg 1997;

http://www-h1.desy.de/ radel/spin99/procsent.html, "Polarized Protons at High Energies - Accelerator Challenges and Physics Opportunities", Workshop, Hamburg, Ed.s A. De Roeck, D. Barber and G. Rädel, DESY-PROC-1999-03 (1999), Hamburg 1999;

"The Spin Structure of the Proton and Polarized Collider Physics", S.D. Bass, A. DeRoeck and A. Deshpande, Nucl. Phys. Vol 105 (2001);

"Transverse Spin Physics", Workshop, Transparencies, DESY-ZEUTHEN-01-01, 374pp, Zeuthen 2001. 
[130] C. Aidala et al. DESY-PROC-1999-03, September 1999, p. 248

[131] Y. Goto et al. (Asymmetry Analysis Collaboration) Phys. Rev. D62 034017 (2000).

[132] M. Glueck, E. Reya, M. Stratmann and W. Vogelsang, Phys. Rev. D63 094005 (2001).

[133] J. Bluemlein and H. Boettcher, Nucl. Phys. B636 225 (2002) [hep-ph/0203155].

[134] A. De Roeck et al. Eur. Phys. J. C6 121 (1999).

[135] J.G. Contreras, A. De Roeck and M. Maul, hep-ph/9711418.

[136] J. Blumlein and D. Robaschik, Phys. Rev. D65 096002 (2002) [arXiv:hep-ph/0202077].

[137] L. Frankfurt, V. Guzey and M. Strikman, Phys. Lett. B381 379 (1996).

[138] J. Edelmann, G. Piller and W. Weise, Z. Phys. A357 129 (1997).

[139] L.L. Frankfurt and M.I. Strikman, Nucl. Phys. A405 557 (1983).

[140] J.G. Contreras, A. De Roeck and M. Maul, "Extraction of $\Delta u$ and $\Delta d$ from CC events using polarised beams at HERA", in Proc. of the 1997 workshop on physics with polarised beams at HERA, Ed.s T. Gehrmann and A. De Roeck, [hep-ph/9711418].

[141] W. van Neerven, in The THERA Book, DESY 01-123F (2001) p. 256.

[142] J.P. Ralston and D.E. Soper, Nucl. Phys. B 152 (109) 1979.

[143] J. Soffer, Phys. Rev. Lett. 741295 (1995).

[144] V. Barone, A. Drago and P.G. Ratcliffe, arXiv: hep-ph/0104283 (2001).

[145] D. Boer, R. Jakob and P.J. Mulders, Nucl. Phys. B564 471 (2000).

[146] M. Stratmann, Proc. of $8^{\text {th }}$ Int. Workshop on DIS, April 2000, Liverpool, England, Ed.s J. Gracey and T. Greenshaw, p. 152.

[147] M. Stratmann, talk at the HERA III workshop at MPI Munich, Dec. 2002, unpublished, see http://wwwhera-b.mppmu.mpg.de/hera3/hera3.html.

[148] R. Jakob, talk in spin session of Hera III Munich Workshop.

[149] P.J. Mulders, "Current fragmentation in semi-inclusive leptoproduction", AIP Conf. Proceedings 588 (2001) 75-88, Proceedings of the 2nd Workshop on Physics with an electron polarized light-ion collider (EPIC2000), Cambridge, Massachusetts, 14-15 Sept 2000, edited by R.G. Milner.

[150] "Future Physics at HERA", Proc. Workshop, DESY 1996, Eds. G. Ingelman, A. De Roeck and R. Klanner (1996) (http://www.desy.de/ heraws96);

S. Levonian, "HERA III", Nucl. Phys. A711 317 (2002). 
[151] A. Bunyatyan et al. "Forward Neutron Calorimeter for H1 experiment at DESY", H112/02-605 (2002);

A. Zhokin, "Different Aspects of the FNC Simulation in the Framework of H1SIM", H107/02-601 (2002).

[152] HERA II optics, private communication, HERA machine group.

[153] C. Adloff et al. [H1 Collaboration] Z. Phys. C76 613 (1997).

[154] R.D. Appuhn et al. Nucl. Instr. Meth. A374 397 (1996).

[155] W. Eick et al. Nucl. Instr. Meth. A386 81 (1997).

[156] R. Horisberger et al. hep-ex/0101023 (2001).

[157] H1 Collaboration, paper 492 presented at EPS, Budapest, 2001

D. Eckstein, Ph.D. Thesis, Berlin, Humboldt University, 2002, available from the H1 web page.

[158] G.H. Hoffstätter, "Electron-ion collisions and other options for HERA", presented at the Durham Workshop on the Future of Lepton-Nucleon Scattering, England, Dec. 2001; available from http://hep.ph.liv.ac.uk/ green/HERAfuture.

[159] E.G. Bessanov and E.V. Tkalya, ArXiv:physics/0212100 v2 (2003).

[160] "Acceleration of Polarized Protons to $820 \mathrm{GeV}$ at HERA", SPIN Collaboration and the DESY Polarization Team, Univ. Michigan UM HE-96-20 (1996).

[161] Update report: "Acceleration of Polarized Protons to $920 \mathrm{GeV}$ at HERA", SPIN Collaboration and the DESY Polarization Team, Univ. Michigan UM HE-99-05 (1999).

[162] D.P. Barber, G.H. Hoffstätter and M. Vogt, Proc. $13^{\text {th }}$ International Symposium on High Energy Spin Physics (SPIN98), Protvino, Russia, 1998, World Scientific (1999).

[163] "Polarized Protons at High Energies - Accelerator Challenges and Physics Opportunities”, Ed.s A. De Roeck, D. Barber and G. Rädel, DESY-PROC-1999-03 (1999).

[164] G.H. Hoffstätter, Habilitation Thesis, Technical University of Darmstadt (2000). Accepted for publication by Springer.

[165] M. Vogt, Ph.D. Thesis, University of Hamburg, DESY-THESIS-2000-054 (2000).

[166] B. Surrow of BNL, private communication, March 2003.

[167] H. Huang et al. Proc. 15 ${ }^{\text {th }}$ International Spin Physics Symposium (SPIN2002), BNL, U.S.A. Sept. 2002.

[168] G.H. Hoffstaetter, $7^{\text {th }}$ European Particle Accelerator Conference (EPAC 2000), Vienna, June 2000.

[169] A. Skrinsky, Proc. of the Workshop EPIC99, Ed. L.C. Bland et al. Bloomington, USA, April 1999, World Scientific. 
[170] Y. Derbenev, private communication;

V. Anferov and Y. Derbenev, Phys. Rev. PRST-AB 12, 1345 (2000).

[171] E.F. Parker et al. IEEE Trans. Nucl. Sci. 263200 (1979).

[172] J. Arvieux, Proc. $6^{\text {th }}$ Int. Symp. on High Energy Spin Physics, Journal de Physique, Colloque C2, Tome 46 (1985).

[173] Yu.K. Pilipenko et al. Proc. $14^{\text {th }}$ International Spin Physics Symposium (SPIN2000), Osaka, Japan, Oct. 2000, AIP 570 (2001).

[174] H. Sato et al. Nucl. Inst. Meth. A385 391 (1997).

[175] V.S. Morozov et al. Proc. $15^{\text {th }}$ International Spin Physics Symposium (SPIN2002), BNL, U.S.A. Sept. 2002.

[176] A. Lehrach, Proc. $15^{\text {th }}$ International Spin Physics Symposium (SPIN2002), BNL, U.S.A. Sept. 2002.

[177] N. Buttimore, Proc. 15 ${ }^{\text {th }}$ International Spin Physics Symposium (SPIN2002), BNL, U.S.A. Sept. 2002. 